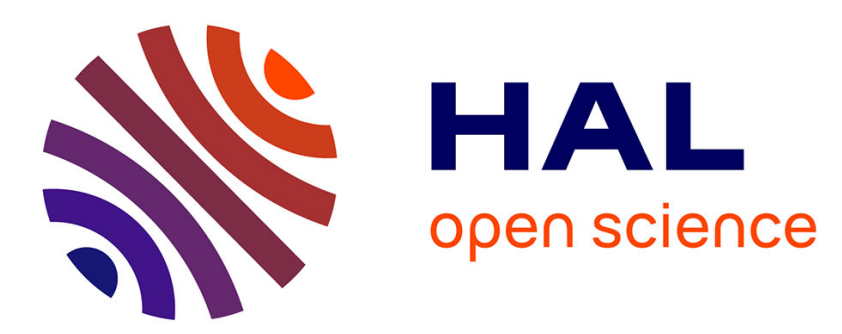

\title{
Experimental Investigation and Large-Eddy Simulation of the Turbulent Flow past a Smooth and Rigid Hemisphere
}

Jens Nikolas Wood, Guillaume de Nayer, Stephan Schmidt, Michael Breuer

\section{To cite this version:}

Jens Nikolas Wood, Guillaume de Nayer, Stephan Schmidt, Michael Breuer. Experimental Investigation and Large-Eddy Simulation of the Turbulent Flow past a Smooth and Rigid Hemisphere. Flow, Turbulence and Combustion, 2016, 97 (1), pp.79-119. 10.1007/s10494-015-9690-5 . hal-01328107

\section{HAL Id: hal-01328107 https://hal.science/hal-01328107}

Submitted on 7 Jun 2016

HAL is a multi-disciplinary open access archive for the deposit and dissemination of scientific research documents, whether they are published or not. The documents may come from teaching and research institutions in France or abroad, or from public or private research centers.

$$
\text { Copyright }
$$

L'archive ouverte pluridisciplinaire HAL, est destinée au dépôt et à la diffusion de documents scientifiques de niveau recherche, publiés ou non, émanant des établissements d'enseignement et de recherche français ou étrangers, des laboratoires publics ou privés. 
Flow, Turbulence and Combustion, vol. 97 (1), pp. 79-119, (2016).

\title{
Experimental Investigation and Large-Eddy Simulation of the Turbulent Flow past a Smooth and Rigid Hemisphere
}

\author{
J. N. Wood, G. De Nayer, S. Schmidt, M. Breuer* \\ Professur für Strömungsmechanik, Helmut-Schmidt-Universität Hamburg, D-22043 Hamburg, Germany
}

\begin{abstract}
The objective of the present paper is to provide a detailed experimental and numerical investigation on the turbulent flow past a hemispherical obstacle (diameter D). For this purpose, the bluff body is exposed to a thick turbulent boundary layer of the thickness $\delta=D / 2$ at $R e=50,000$. In the experiment this boundary layer thickness is achieved by specific fences placed in the upstream region of the wind tunnel. A detailed measurement of the upstream flow conditions by laser-Doppler and hot-film probes allows to mimic the inflow conditions for the complementary large-eddy simulation of the flow field using a synthetic turbulence inflow generator. These clearly defined boundary and operating conditions are the prerequisites for a combined experimental and numerical investigation of the flow field relying on the laserDoppler anemometry and a finite-volume Navier-Stokes solver for block-structured curvilinear grids. The results comprise an analysis on the unsteady flow features observed in the vicinity of the hemisphere as well as a detailed discussion of the time-averaged flow field. The latter includes the mean velocity field as well as the Reynolds stresses. Owing to the proper description of the oncoming flow and supplementary numerical studies guaranteeing the choice of an appropriate grid and subgrid-scale model, the results of the measurements and the prediction are found to be in close agreement.
\end{abstract}

Keywords: hemisphere; hemispherical dome; turbulent flow; laser-Doppler anemometry; constant temperature anemometry; large-eddy simulation (LES); wind load; artificial boundary layer; inflow generator

\section{Introduction}

Flow fields around surface-mounted bluff bodies in turbulent boundary layers are of common interest in environmental and civil engineering as they appear in various applications such as presented in Fig. 1. Spherically shaped objects such as domed structures exhibit very complex flow pattern that can be roughly classified into an upstream horseshoe vortex system and a recirculation area with trailing vortices in the wake region. The present study includes experimental investigations and large-eddy simulations (LES) to characterize the three-dimensional flow field around a surface-mounted smooth hemisphere in a turbulent boundary layer. A brief epitome of the literature shall provide an overview of the key aspects summarizing the fundamental experimental examinations that were conducted on flows around hemispheres. This part is sub-divided into measurements concerning the pressure distribution, the mean flow and

\footnotetext{
${ }^{*}$ Corresponding author

Email address: breuer@hsu-hh.de (M. Breuer)
} 
the visualization of flow structures. Subsequently, the conducted numerical simulations and studies which combine experimental and numerical investigations are highlighted.

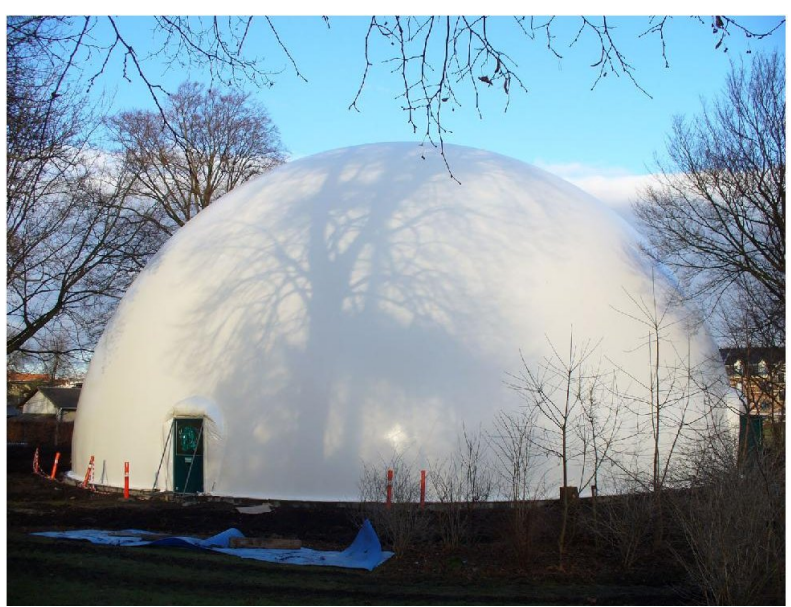

(a) Air inflated structure in Gentofte (Denmark) ${ }^{1}$.

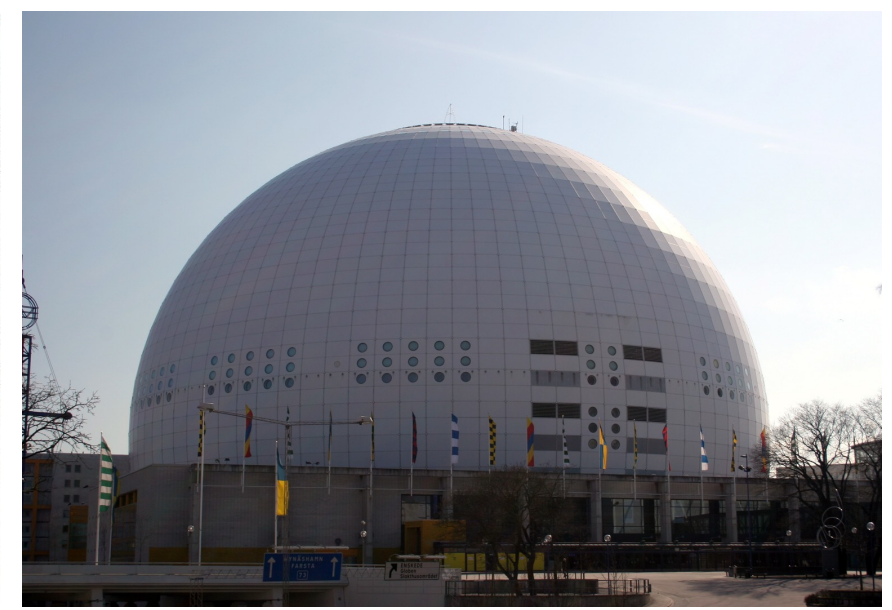

(b) Stockholm Globe Arena (by Tage Olsin CC BY-SA 2.0).

Figure 1: Examples of hemispherical domes in modern civil engineering.

Beginning with an analysis based on the pressure distribution the first traceable experiment was carried out by Jacobs [1] in 1938. The measurements focused on the effects of surface roughness caused by a small hemispherical rivet. Later on Maher [2] carried out investigations on a series of hemispheres that were placed on the ground of a wind tunnel in a boundary layer. After exceeding a Reynolds number ${ }^{2}$ of $\mathrm{Re}=1.6 \times 10^{6}$ the surface drag coefficient showed no further variations due to supercritical flow conditions. Similar results were observed in a comprehensive study by Taylor [3] confirming the effect of the Reynolds number independency after exceeding $\operatorname{Re}=2 \times 10^{5}$ and additionally surpassing a turbulence intensity of $4 \%$. Another experiment by Taniguchi et al. [4] found that there is a relationship between the approaching boundary layer thickness and the aerodynamic forces acting on the hemisphere. Furthermore, Cheng et al. [5] conducted intensive surface pressure measurements in a specialized boundary layer wind tunnel. The turbulent boundary layer featured suburban flow field characteristics with large turbulence intensities ranging between $18 \%$ and $25 \%$. The Reynolds numbers for the turbulent conditions varied between $\operatorname{Re}=5.3 \times 10^{4}$ and $1.7 \times 10^{6}$ depending on the size of the hemisphere. The outcome encourages the previous observations made by Maher [2] and Taylor [3].

Besides focusing on pressure measurements Toy et al. [6] initially investigated the flow field past a hemispherical dome using hot-wire and pulsed-wire anemometers. Based on this investigation Savory and Toy [7] brought a yet deeper insight into the complex flow structures occurring in the near-wake regime of hemispheres and cylinders with hemispherical caps that were exposed to three different turbulent boundary layers within the range from $\operatorname{Re}=4.31 \times 10^{4}$ to $1.4 \times 10^{5}$. The investigation included the effects of surface roughness of the model on the drag coefficient as well as the velocity field of the recirculation area past the hemisphere. A second experiment conducted by Savory and Toy [8] focused on the separation of the shear layer in the flow around hemispheres conducted at a sub-critical Reynolds number of $\operatorname{Re}=1.4 \times 10^{5}$. The study included different turbulent boundary layers classified in thin, smooth and rough boundary layers depending on the thickness and the turbulence intensity. To generate the desired

\footnotetext{
${ }^{1}$ http://www.texlon.ch/en/projects/air-inflated-structures.php

${ }^{2}$ All mentioned Reynolds numbers are based on the diameter of the hemisphere.
} 
Flow, Turbulence and Combustion, vol. 97 (1), pp. 79-119, (2016).

inflow characteristics, Savory and Toy applied artificial boundary layer installations including fences and vortex generators in order to investigate the influence of the upstream boundary conditions. A profound discussion provided a deeper understanding of the distribution of the turbulent shear stress and intensity in the wake regime. Additionally, a representative illustration of the flow field characteristics was made that includes the horseshoe vortex system, the trailing vortices and the separation regions. The outcome of both studies $[7,8]$ is often used as reference for experimental and numerical examinations. A comparable schematic view is provided by Martinuzzi and Tropea [9] in case of the flow past a wall-mounted cube, as well as by Pattenden et al. [10] for the flow around a wall-mounted cylinder. Indeed, both cases possess similar separation and reattachment characteristics.

Primary visualizations of the vortical flow structures were conducted by Tamai et al. [11]. The experimental setup included two hemispheres of different size exposed to water flow in the range $2 \times 10^{2}<\operatorname{Re}<1.2 \times 10^{4}$. The experiments allowed to visualize the complex vortical structures by injecting dye into the water channel. Moreover, the frequencies of the vortex formation and shedding from the separation area were recorded by measuring the spectra of the velocity fluctuations inside and outside the recirculation zone. Another observation was made by Acarlar and Smith [12] who carried out elaborate experiments using relatively small hemispheres in the laminar flow regime to generate hairpin vortices. It turned out that the downstream velocity profiles resulting from the artificially induced flow structures were similar to those of a turbulent boundary layer. Bennington [13] examined various roughness elements and their associated effects on the turbulent boundary layer. Among the chosen elements, a hemispherical obstacle is analyzed in detail concerning statistics of the Reynolds stresses, the turbulent kinetic energy and even the triple correlations.

Simpson et al. [14] examined the flow separation at an axisymmetric bump by utilizing surface mean pressure measurements, oil flow visualizations and laser-Doppler measurements. The results showed a nearly symmetric mean flow over the bump including a detailed mapping of separation and nodal points on the leeside of the obstacle. Furthermore, Byun and Simpson [15] intensified the research on the bump to characterize the 3D separations by using a fine-spatial-resolution laser-Doppler system and later [16] supplemented the studies by adding an investigation on the pressure fluctuations. Similar to the flow past the hemisphere a pressure-driven separation occurs. However, the separation line is shifted further downstream and reattachment is much earlier, leading to a smaller recirculation area.

Further visualization experiments were conducted by Yaghoubi [17]. They comprised a detailed visualization of the air flow pattern around grouped hemispheres in a wind tunnel. The motivation for the study was to achieve a deeper understanding of the flow field and the associated effects of natural ventilation of domed structures often appearing in oriental architecture.

Apart from experimental investigations numerical simulations were carried out to provide enhanced insight into the flow. An early study was conducted by Tamura et al. [18] without applying any turbulence model. The focus of the simulations lay on the visualization of the unsteady flow pattern and the time-averaged surface pressure distribution at $\operatorname{Re}=2 \times 10^{3}$ and $2 \times 10^{4}$, respectively.

A fundamental numerical study was carried out by Manhart [19] using large-eddy simulation to receive more detailed information about the vortical structures at $\operatorname{Re}=1.5 \times 10^{5}$. The Cartesian grid combined with the immersed boundary technique led to an artificial surface roughness on the contour of the hemisphere. The results were therefore compared with the experiments of Savory and Toy $[7,8]$ for a rough hemisphere. Besides observations of temporal spectra and the velocity distributions, the proper orthogonal decomposition method was applied to examine the highly complex separation processes and to determine the dominant 
Flow, Turbulence and Combustion, vol. 97 (1), pp. 79-119, (2016).

vortical structures.

Another comparison of numerical and experimental data was made by Meroney et al. [20]. The three-dimensional wind load distributions on smooth, rough and dual domes in the shape of hemispherical caps were examined. The calculations were carried out for $\operatorname{Re}=1.85 \times 10^{5}$ and $1.44 \times 10^{6}$. Several RANS turbulence models including the classical k- $\epsilon$ model, a Reynolds stress model [21] and the Spalart-Allmaras model [22] were used delivering similar results. Recently, Kharoua and Khezzar [23] performed a LES on a hemisphere with a rough and smooth surface at $\mathrm{Re}=1.4 \times 10^{5}$ comparing the results with the experiment of Savory and Toy [7]. A specialized approach to model the surface roughness was presented. The results of the LES allowed the visualization of instantaneous three-dimensional flow pattern illustrating the complex interaction of vortical structures in the close vicinity of the hemisphere. It turned out that the model roughness leads to a larger recirculation area compared to the smooth surface.

García-Villalba et al. [24] conducted LES to study the behavior of turbulent flow separation from an axisymmetric three-dimensional bump at a Reynolds number of $\operatorname{Re}=1.3 \times 10^{5}$. The characteristics of the turbulent flow field were compared with the experimental results mentioned above [14-16] strongly focusing on the formation of the separation region on the rear side of the bump.

A combined experimental and numerical study was accomplished by Tavakol et al. [25]. A hemisphere was immersed in two turbulent boundary layers of different thickness. The experiments were conducted in a wind tunnel using a hot-wire sensor to record the velocity field at certain planes upstream and downstream of the hemisphere at $\operatorname{Re}=6.4 \times 10^{4}$. Velocity distributions and turbulence intensities were presented for the streamwise and the spanwise directions in the recirculation zone. A further velocity measurement was carried out for the area close to the front of the hemisphere investigating the horseshoe vortex that leads to a strong backflow in the near-wall region. The numerical investigation relied on the RNG k- $\epsilon$ turbulence model [26]. The inflow conditions of the simulation were generated by implying the time-averaged data of the corresponding hot-wire measurements. The turbulence intensity at the inlet is also taken from the measurements. The results showed overall good agreement with the experimental data. Recently, Tavakol et al. [27] presented a yet deeper investigation of the hemisphere flow using LES at Re $=3.6 \times 10^{4}$ and $6.4 \times 10^{4}$. Based on the earlier study [25] the main focus of this paper was to highlight the superior results of the applied LES compared to the previously performed RANS simulations. The numerical grid consisted of $4.2 \times 10^{6}$ CVs. A detailed analysis of different subgrid-scale (SGS) models, i.e., WALE [28], dynamic Smagorinsky [29] and the kinetic energy transport model [30] was performed. The study included a thin turbulent boundary layer $\delta / D \approx 0.15$ as inflow condition. For a realistic inlet velocity distribution including fluctuations a turbulence inflow generator based on the method of Sergent [31] was applied. As a result the LES showed excellent agreement with the measurements. An updated comparison between the previous study [27] with the current data revealed the shortcomings of the RNG k- $\epsilon$ model especially in the wake of the hemisphere. A presentation of time-averaged data focuses on the streamline visualization and surface pressure distribution. Unfortunately, the paper does not present statistical data of the velocity field or the Reynolds stresses.

The literature presented indicates that a surface-mounted hemisphere in a turbulent boundary layer reveals a very complex flow field. The key aspect of most studies often remains on one specific issue such as the recirculation area or the pressure distribution. Just a few studies contain general characteristics of the flow including complementary numerical and experimental investigations. 
Flow, Turbulence and Combustion, vol. 97 (1), pp. 79-119, (2016).

The present study focuses on the following objectives: Firstly, it shall provide a comprehensive view of the flow field past a hemispherical object immersed in a turbulent boundary layer at $\mathrm{Re}=50,000$ with the help of experimental/numerical investigations: All relevant regions of the flow field (horseshoe vortex system, recirculation area and wake) have to be studied in detail including unsteady characteristics such as vortex shedding and related spectral analysis. Secondly, the knowledge gained from the present examination of the rigid structure is important for the upcoming investigations on fluid-structure interaction. Finally, the complementary experimental/numerical test case of the surface-mounted hemisphere shall offer a novel benchmark for the evaluation and validation of numerical schemes or new turbulence models.

The structure of the paper is as follows: Section 2 presents the general description of the case including the basic parameters. The experimental setup is outlined in Section 3 followed by the numerical approach in Section 4. The comparison of the experimental and numerical results is sub-divided into the unsteady flow characteristics in Section 5 and the time-averaged data in Section 6 including an overall discussion. Finally, conclusions are drawn in Section 7. For the sake of clarity, the numerical investigations on the influence of the subgrid-scale (SGS) model and of the synthetic turbulence inflow data are shifted to the Appendix.

\section{Description of the case}

The purpose of the present study is to investigate the flow around a surface-mounted hemisphere placed in a turbulent boundary layer. Figure 2 depicts the investigated case. The rigid hemispherical body (diameter $D$ ) is mounted on a smooth wall. The surface of the hemisphere is also considered to be ideally smooth. The structure is put into a thick turbulent boundary layer which can be described by a $1 / 7$ power law as reviewed by Couniham [32]. At a distance of 1.5 diameters upstream of the bluff body the thickness of the boundary layer $\delta$ corresponds to the height of the hemisphere, i.e., $\delta=D / 2$. The Reynolds number of the air flow $\left(\rho_{\text {air }}=1.225 \mathrm{~kg} / \mathrm{m}^{3}, \mu_{\text {air }}=18.27 \times 10^{-6} \mathrm{~kg} /(\mathrm{m} \mathrm{s})\right.$ at $\left.\vartheta=20^{\circ} \mathrm{C}\right)$ is set to $\operatorname{Re}=\rho_{\text {air }} D U_{\infty} / \mu_{\text {air }} \approx$ $50,000 . U_{\infty}$ is the undisturbed free-stream mean velocity in $x$-direction outside the boundary layer at standard atmospheric conditions. The Mach number is low (Ma $\leq 0.03)$. At this Mach number the air flow can be assumed to be incompressible. Moreover, the fluid is considered to be isotherm.

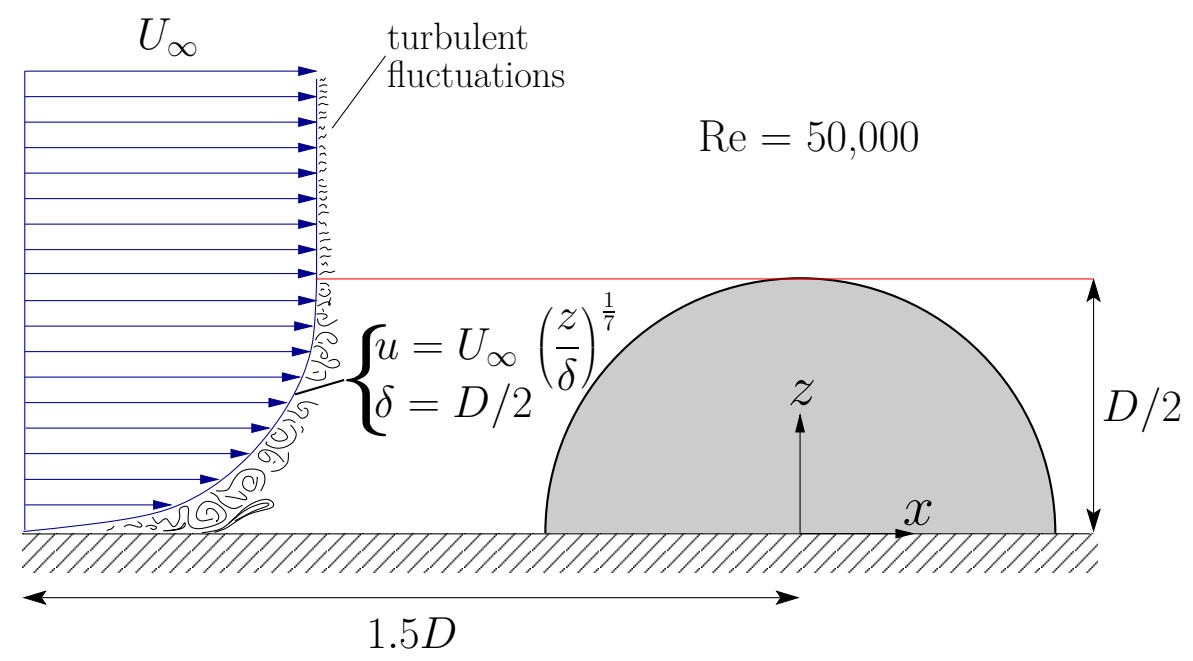

Figure 2: Surface-mounted hemisphere within a turbulent boundary layer. 
Flow, Turbulence and Combustion, vol. 97 (1), pp. 79-119, (2016).

In the present paper all quantities will be given in dimensionless form using the diameter of the hemisphere $D$, the undisturbed free-stream velocity $U_{\infty}$ and the fluid density $\rho_{\text {air }}$. The origin of the frame of reference is taken at the center of the base area of the hemisphere, where $x$ denotes the streamwise, $y$ the spanwise and $z$ the vertical direction (wall-normal).

\section{Experimental setup and measuring equipment}

This section is divided into three parts. First, the wind tunnel and the experimental settings are introduced in detail. Besides the basic features, special settings and manufacturing issues are presented. This is followed by a brief overview of the instrumentation applied. Finally, the method for artificial boundary layer thickening is presented, since this is a key aspect of the flow field.

\subsection{Wind tunnel and test setup}

The experimental investigations for the flow past the hemisphere are carried out in a Göttingentype subsonic wind tunnel with an open test section presented in Fig. 3. Further design specifications are summarized in Table 1.

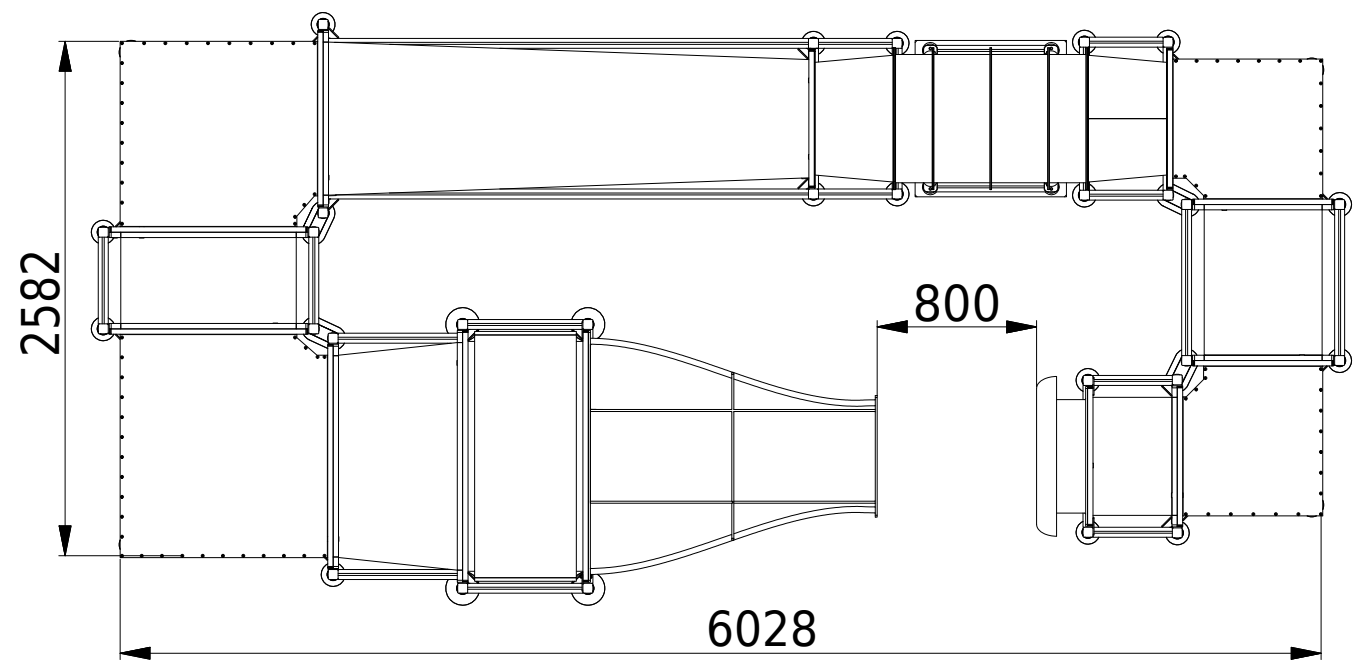

Figure 3: Wind tunnel applied for the experimental investigations.

Table 1: Design specifications of the wind tunnel and test setup.

\begin{tabular}{|c|c|}
\hline feature & dimension \\
\hline \hline nozzle cross-section & $375 \mathrm{~mm} \times 500 \mathrm{~mm}$ \\
\hline length of test section & $800 \mathrm{~mm}$ \\
\hline nozzle contraction ratio & $5: 1$ \\
\hline velocity range & $5 \mathrm{~m} / \mathrm{s}-28 \mathrm{~m} / \mathrm{s}$ \\
\hline free-stream streamwise turbulence intensity $\left(\mathrm{Tu}_{u}^{0}\right)$ & $<0.2 \%$ \\
\hline flat plate & $600 \mathrm{~mm} \times 620 \mathrm{~mm}$ \\
\hline diameter of the hemisphere & $150 \mathrm{~mm}$ \\
\hline
\end{tabular}

The hemisphere is placed on a flat smooth plate which is mounted onto a table. The dimensions based on the diameter of the hemisphere and the position of the model in the test section are illustrated in Fig. 4(a) with reference to the symmetry plane of the setup. The leading edge of 
Flow, Turbulence and Combustion, vol. 97 (1), pp. 79-119, (2016).

the plate is in alignment with the bottom of the rectangular nozzle of the wind tunnel. This is necessary to transfer the artificially thickened boundary layer from the nozzle to the test section without any gaps. The flat plate is designed to cover the complete spanwise extension of the cross-section of the wind tunnel in order to ensure a smooth transition of the near-wall flow from the nozzle to the test section. Thus, a gap remains between the trailing edge of the flat plate and the receiver. The actual setup is depicted in Fig. 4(b).

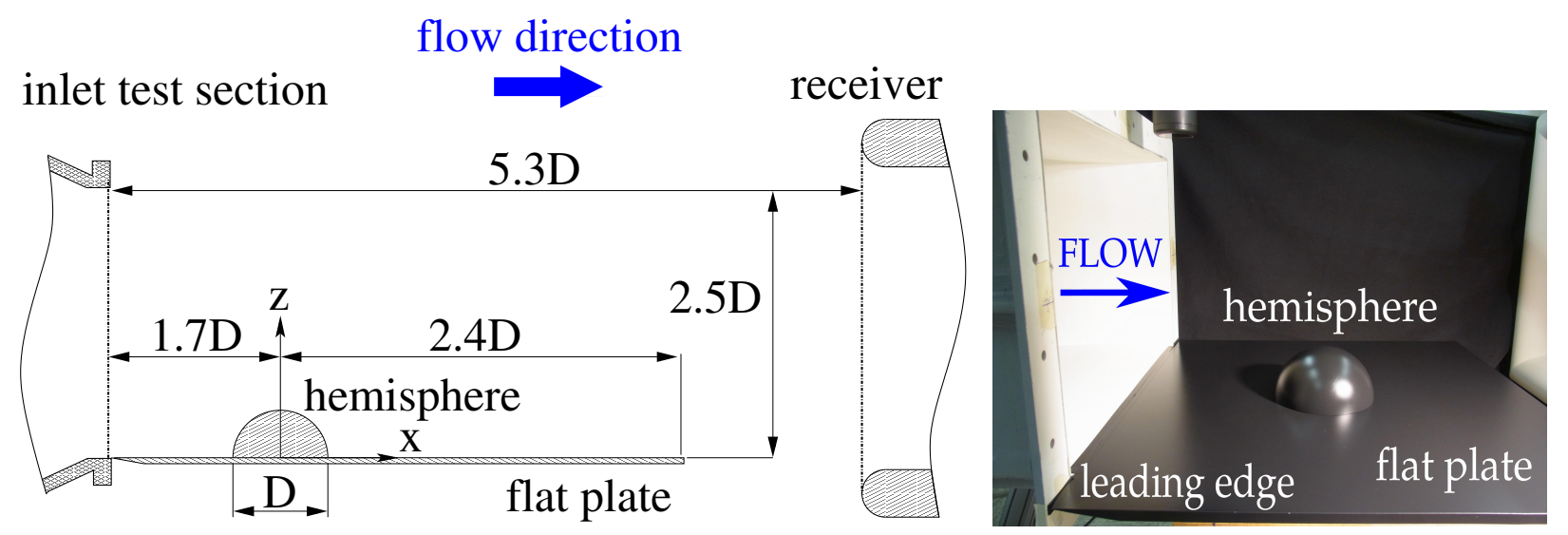

(a) Schematic illustration of the dimensions based on the diameter.

(b) Actual setup.

Figure 4: Dimensions and position of the hemisphere in the test section.

The model of the hemisphere as well as the flat plate are made of an aluminum alloy. The surface is additionally varnished with black paint to minimize reflections of the laser light during measurements close to the surface. An overall average roughness on the contour of $\mathrm{R}_{a}<0.8 \mu \mathrm{m}$ is achieved. The diameter of the hemisphere is $D=150 \mathrm{~mm}$. To ensure the rigidity of the model, it is designed as a solid block without any cavities. The blocking ratio of the hemisphere in the wind tunnel is approximately $4.7 \%$ based on the projected area $A_{\text {hemi }}=(\pi / 8) D^{2}$ of the hemisphere and the area of the cross-section of the nozzle. To adjust the Reynolds number to $\mathrm{Re}=50,000$, the blower of the wind tunnel is set to a free-stream velocity of $U_{\infty}=5.14 \mathrm{~m} / \mathrm{s}$. For an empty test section the free-stream streamwise turbulence intensity $\mathrm{Tu}_{u}^{0}=\left(u^{\prime}\right)_{r m s} / U_{\infty}$ is less than $0.2 \%$. This value is based on high resolution single-wire constant temperature anemometry (CTA) measurements that were conducted to specify the overall quality of the wind tunnel. According to LDA measurements the free-stream spanwise and wall-normal turbulence intensities are one order of magnitude smaller, which leads to a free-stream total turbulence level of $\mathrm{Tu}_{t o t}^{0}=\sqrt{\frac{1}{3}\left(\overline{u^{\prime 2}}+\overline{v^{\prime 2}}+\overline{w^{\prime 2}}\right)} / U_{\infty} \approx 0.1 \%$.

\subsection{Measurement equipment used for flow analysis}

\subsubsection{Configuration of the laser-Doppler anemometer}

The flow past the hemisphere is measured by a non-invasive 2D laser-Doppler anemometer offering high resolution velocity measurements of all components, $u$ (streamwise), $v$ (spanwise) and $w$ (wall-normal direction), respectively. The system consists of the following components: The laser beam is generated by a water-cooled Coherent Innova 70C argon-ion laser. The beam is guided to a Dantec FiberFlow transmitter box, where it is split up into two different wave lengths (514.5 nm and $488 \mathrm{~nm}$ ) which are used for each velocity component. The beams are sent from the transmitter box to a $2 \mathrm{D}$ optical probe. In order to receive information about all three velocity components the LDA probe is configured in two ways: In configuration 1 the 
Flow, Turbulence and Combustion, vol. 97 (1), pp. 79-119, (2016).

probe is facing the flat plate so that the optical axis of the laser beam points vertically at the surface of the plate as depicted in Fig. 5(a). The setup is primarily used to record the spanwise component $v$. The illustration shows the instantaneous flow field in the symmetry $x-z$-plane of the present study. The dashed area in blue indicates the measuring plane. The associated experimental grid used for the LDA measurements is highlighted in Fig. 5(c). The distribution of the points is chosen based on the characteristics of the predicted flow field. In the near-wall region and the recirculation area close to the hemisphere more measurement points are placed to ensure a good resolution of the gradients.

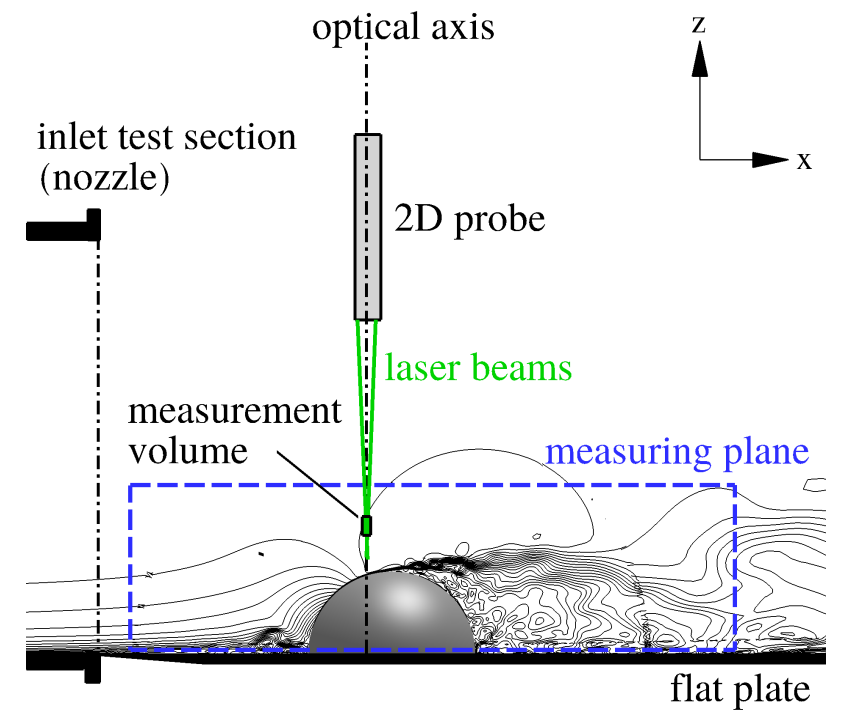

(a) Configuration 1 of the LDA system.

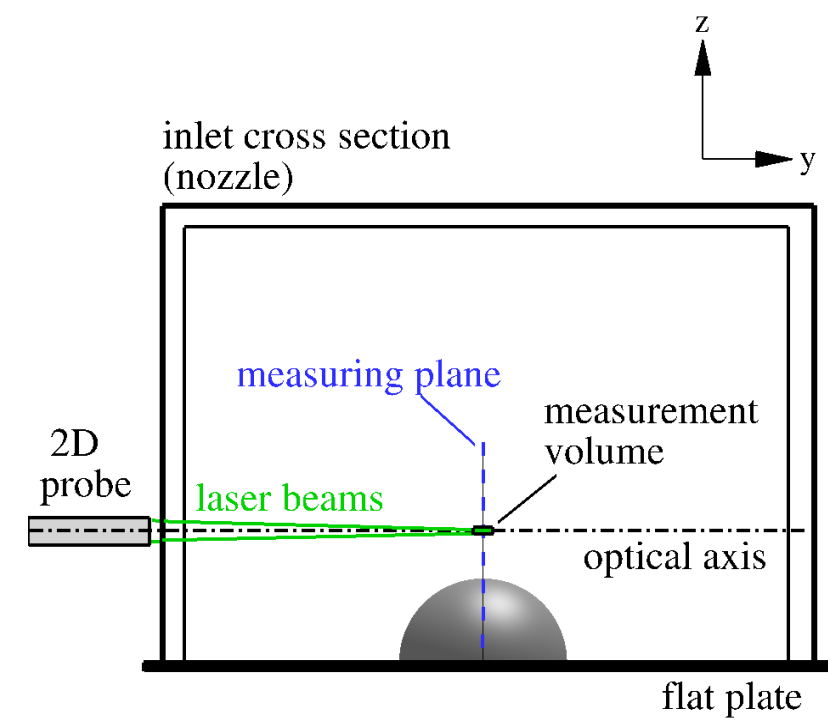

(b) Configuration 2 of the LDA system.

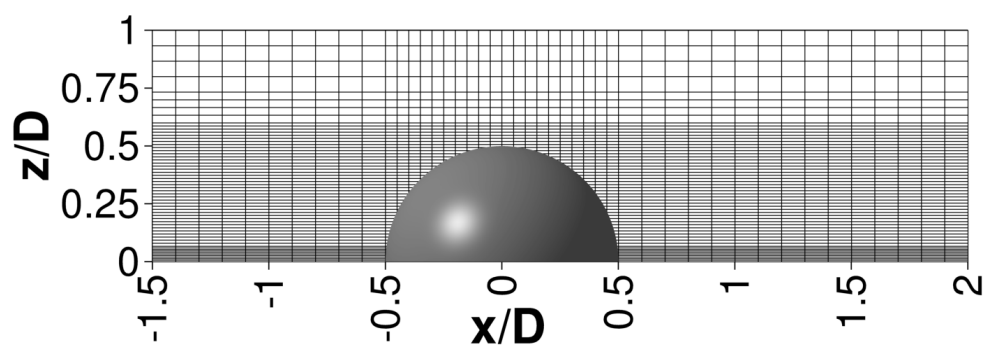

(c) LDA grid in the symmetry plane (2041 measurement points).

Figure 5: LDA configuration and measurement grid resolution of the symmetry $x-z$-plane.

Configuration 2 is shown in Fig. 5(b) and is utilized to acquire the streamwise and wall-normal flow components. In this picture the spanwise $y-z$-plane shows the measuring plane as a blue dashed line since the whole view point is rotated by $90^{\circ}$ compared to configuration 1 . In this setup the near-wall region of the streamwise component $u$ has a higher spatial resolution as realizable in configuration 1 due to the orientation of the optical axis parallel to the plate. That avoids unwanted laser reflections of the surface that would otherwise point directly at the photo multipliers of the LDA probe. However, the measurement of the wall-normal component $w$ is geometrically restricted by the crossing angle of the laser beam. When the probe moves closer to the surface, a certain position is reached, i.e., $13 \mathrm{~mm}$ above the surface, where the lower laser beam hits the edge of the flat plate and is blocked. Thus, in the near-wall region the flat plate acts as an optical barrier that interrupts the formation of the measuring volume. Consequently, no data can be recorded for the wall-normal component below this particular separation point. The measurements and the positioning of the traverse are operated by the 
Flow, Turbulence and Combustion, vol. 97 (1), pp. 79-119, (2016).

Dantec BSA Flow Software ${ }^{3}$. Based on both configurations the three-dimensional flow field within the chosen $x-z$-measuring plane around the hemisphere can be determined with the mentioned restrictions for $w$.

To investigate the flow behind the hemisphere complementary measurements are achieved in the $y$-z-plane $(-1 \leq y / D \leq 1$ and $0 \leq z / D \leq 1)$ at $x / D=0.5$. Configuration 1 is used to measure the streamwise and spanwise velocity components on a grid with 1239 measurement points.

The aerosol generator TSI Six-Jet Atomizer 9306 is utilized to generate small droplets of Di-Ethyl-Hexyl-Sebacat (DEHS) with an average size between 0.2 and $0.3 \mu \mathrm{m}$. The spherical droplets feature a long-life cycle and excellent optical properties for laser-Doppler anemometry. The droplets are atomized and seeded at the receiver of the wind tunnel.

\subsubsection{Complementary constant temperature anemometer measurements}

In addition to the laser-Doppler anemometry a constant temperature anemometer (TSI 1750 CTA) is utilized to record the velocity spectra in the wake of the hemisphere. The spectra shall provide information about the driving vortex shedding processes and their related frequencies as well as the overall decay in the inertial subrange. The main reason to use CTA for this specific analysis instead of LDA is that raw data samples from laser-Doppler measurements show an irregular time signature since the measured droplets pass the measurement volume randomly. Commonly the data are resampled by using a sampling and holding algorithm [3335 which transfers the random time signal into an equidistant data set. Nevertheless, the resampling has a great impact on the calculation of the power spectra of flows with limited concentration of droplets like air flow in wind tunnels, which leads to a lower sampling rate. According to Adrian and Yao [33] it acts as a first-order low-pass filter. The low-pass filter frequency is approximately $f_{c o}=\dot{n} / 2 \pi$, where $\dot{n}$ is the average data rate per second. The measurements of the present LDA study involve average data rates of about $1 \mathrm{kHz}$ in the freestream. Approaching the wall a decrease of the data rate is detected which leads to a minimum sampling of about $0.2 \mathrm{kHz}$ in the near-wall region. As a consequence of the moderate sampling rate a low cut-off frequency in the range of $64 \leq f_{c o} \leq 158 \mathrm{~Hz}$ results due to resampling. Thus, in the present case, it is not desirable to use resampled data as it will contain filtered information above the cut-off frequency leading to an attenuated spectral decay.

The CTA system is also used as a secondary device to validate the LDA data of the artificial turbulent boundary layer as it is described in the next section.

\subsection{Generation of the artificial turbulent boundary layer}

This paragraph presents the methods for artificially adjusting the oncoming boundary layer to fit to the inlet conditions briefly described in Section 2. The test case assumes an upstream velocity profile that is based on the $1 / 7$ power law of a turbulent boundary layer. This particular velocity distribution is utilized at the beginning of the test section to guarantee a clearly defined boundary layer and thus to achieve comparability between the experimental investigations and the numerical simulations. The test section of a wind tunnel is often not long enough to generate naturally developing turbulent boundary layers of a desired thickness. To satisfy the specifications of the test case, the setup of the wind tunnel has to be modified to achieve the proper boundary layer thickness. In the current case the thickness of the boundary layer is specified to be equal to the height of the hemisphere $\delta /(D / 2)=1$ at a distance of $x / D=-1.5$ upstream of the hemisphere. This can only be achieved by modifying the bottom of the wall

\footnotetext{
${ }^{3}$ http://www dantecdynamics. com/bsa-flow-software-for-lda-and-pda
} 
Flow, Turbulence and Combustion, vol. 97 (1), pp. 79-119, (2016).

inside the nozzle by installing turbulence generators that are used to artificially thicken the height of the boundary layer over a short distance. In summary, a preliminary objective of the present study is to ensure that the generated turbulent boundary layer corresponds to the predefined conditions.

In the past, detailed studies [36-38] were conducted regarding artificial boundary layer thickening to mimic the influence of atmospheric conditions in near-ground flow. Parts of these investigations are used in the present experimental setup and shall be discussed briefly. An early compendium of different methods including obstacles such as gauzes, rods and flat plates to produce velocity profiles in wind tunnels was summarized by Lawson [36]. At the same time Counihan [37] presented a setup consisting of a castellated barrier followed by elliptic wedges that work as vortex generators. As a result, these devices were capable to simulate the velocity distribution similar to a natural turbulent boundary layer when placed at a distance equal to about four to five desired boundary layer heights upstream of the test section. Furthermore, Sargison et al. [38] provided a review of three devices including a fence with triangular spikes and two plates with different holes to increase the thickness of boundary layers in a wind tunnel. All three measures are suitable to enlarge the boundary layer thickness.

In a preliminary study investigating the development of the boundary layer on the flat plate (without the hemisphere placed in the test section) a combination of these devices was tested including the methods mentioned above. Due to the restricted dimensions of the nozzle of the wind tunnel and the test section, the modeling space, where the vortex generators can be placed, is geometrically limited. The challenge of artificial boundary layer design is the generation of thick boundary layers over relatively short distances avoiding unwanted persisting vortical structures that can result from inappropriate devices. Therefore, an intensive analysis of the behavior of each turbulence generator led to the optimum combination of these devices to achieve the desired boundary layer profile within the given working space. As a final outcome, a fence with equidistant rods followed by a castellated barrier and a fence with triangular spikes is chosen to mimic the influence of an atmospheric turbulent boundary layer. A schematic representation of the setup and its functionality is depicted in Fig. 6.

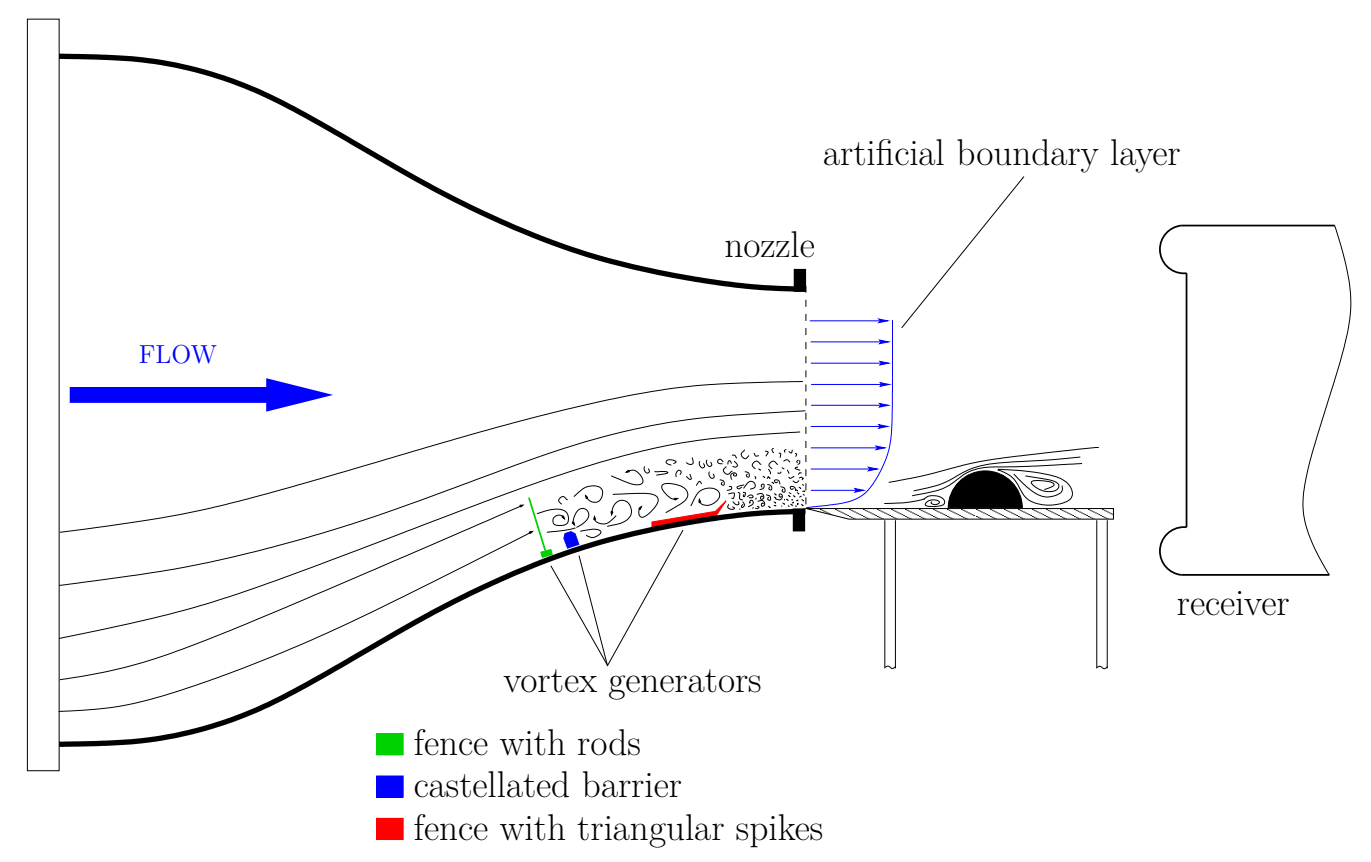

Figure 6: Turbulence generators mounted onto the bottom wall of the wind tunnel's nozzle. 
Flow, Turbulence and Combustion, vol. 97 (1), pp. 79-119, (2016).

It shows the symmetry plane of the complete nozzle. Near the outlet the three mentioned vortex generators are mounted onto the bottom wall of the nozzle. The first vortex generator is the fence with rods highlighted in green. It is closely followed by a castellated barrier marked in blue. Both devices are used to generate large disturbances in the near-wall flow. The produced vortical structures travel to the third vortex generator illustrated in red, which is the fence with triangular spikes. It is used to break up the large structures for a more isotropic turbulence distribution in the boundary layer. The exact positions of the vortex devices can be found in Appendix Appendix A.

Corresponding measurements of the inlet boundary layer profile including the mean velocity and the turbulent fluctuations are depicted in Fig. 7. For the evaluation of the artificial turbulent boundary layer the streamwise component $u$ is additionally measured by a specialized constant temperature platinum film probe (TSI model 1218) along with a TSI 1750 constant temperature anemometer. This method is used as a secondary data source for the streamwise component with special emphasis on the near-wall region. It is directly compared to the measurements by the laser-Doppler anemometry setup (configuration 2, see Fig. 5(b)) which has certain restrictions close to the surface of the plate (see Section 3.2.1).

All data are recorded just after the beginning of the test section at $x / D=-1.5$ in the symmetry plane without placing the hemisphere into the test section. As a reference velocity distribution the $1 / 7$ power law with $\delta=D / 2$ is used to evaluate the measurements. The power law and the constant free-stream velocity above $z / D=0.5$ is illustrated in Fig. 7 (a) as a blue line. The measured mean velocity distribution $\bar{u} / U_{\infty}$ is in close agreement with the reference exhibiting minor deviations in the region $z / D=0.25$. The free-stream velocity $\bar{u} / U_{\infty} \approx 1$ is reached at about $z / D \approx 0.5$ which indicates that the desired thickness of the boundary layer is attained. Additionally, the displacement thickness $\delta_{1} / \delta$ and the momentum thickness $\delta_{2} / \delta$ are evaluated from the experimental data to $1 / 8$ and $7 / 72$, respectively. It leads to a shape factor of $H=\delta_{1} / \delta_{2}=1.286$ which confirms a classical property of a turbulent boundary layer. The Reynolds number based on $\delta_{2}$ is estimated to $\operatorname{Re}_{\delta_{2}}=2503$.

Figure 7(b) presents the dimensionless velocity $u^{+}$plotted against the dimensionless wallnormal distance $z^{+}$. The first measured point is located at a distance of $\Delta z=0.25 \mathrm{~mm}$ above the flat plate. The velocity distribution is nearly linear in the region $4 \leq z^{+} \leq 10$. Therefore, the first two points close to the wall are still inside the viscous sublayer. This is in good agreement with the literature which often states the border between the viscous sublayer and the buffer layer at about $z^{+} \approx 5$. The friction velocity is estimated to $u_{\tau}=\sqrt{\tau_{w} / \rho_{\text {air }}}=0.225 \mathrm{~m} / \mathrm{s}$ $\left(u_{\tau} / U_{\infty}=4.38 \times 10^{-2}\right)$, where $\tau_{w}$ is approximated by $\mu_{\text {air }} \Delta u / \Delta z$. Both classical laws of the wall (viscous sublayer: $u^{+}=z^{+}$and log-layer: $u^{+}=1 / 0.41 \ln \left(z^{+}\right)+5.2$ ) are correctly reproduced. Some discrepancies in the velocity distribution are observed in the log area. Indeed, the measured boundary layer does not exactly follow the $1 / 7$ power law. A comparison between the LDA and CTA data shows only minor differences which means that the LDA system is capable to achieve the required near-wall resolution for the chosen setup. Furthermore, the distribution of the mean velocity across the spanwise expansions of the test section shows only small variations (not shown here for the sake of brevity).

The turbulent fluctuations $\left(u^{\prime}\right)_{r m s} / U_{\infty},\left(v^{\prime}\right)_{r m s} / U_{\infty}$ and $\left(w^{\prime}\right)_{r m s} / U_{\infty}$ are given in Fig. $7(\mathrm{c})$ in percent. It is obvious that the streamwise fluctuations recorded by LDA and CTA are very similar. Therefore, it is assumed that the velocity components $v$ and $w$ which are solely measured by LDA, are also valid. This is supported by the fact that Counihan [37] measured a similar distribution of the turbulence intensities in the case of the elliptic turbulence generators. Furthermore, similar distributions were also obtained by Schlatter et al. [39] based on a direct numerical simulation (DNS) of a turbulent boundary layer flow on a flat plate at a Reynolds 


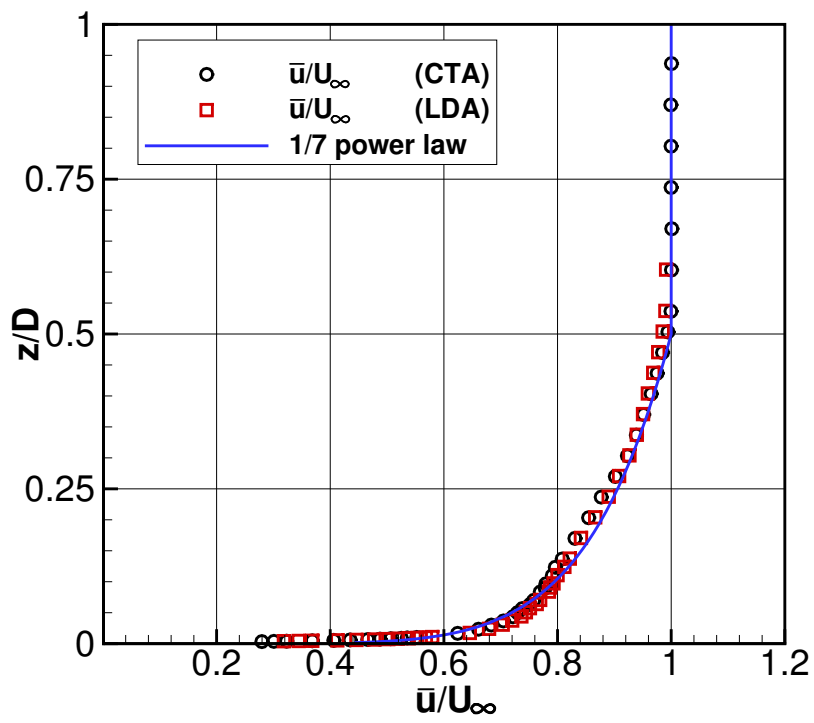

(a) Streamwise mean velocity profile.

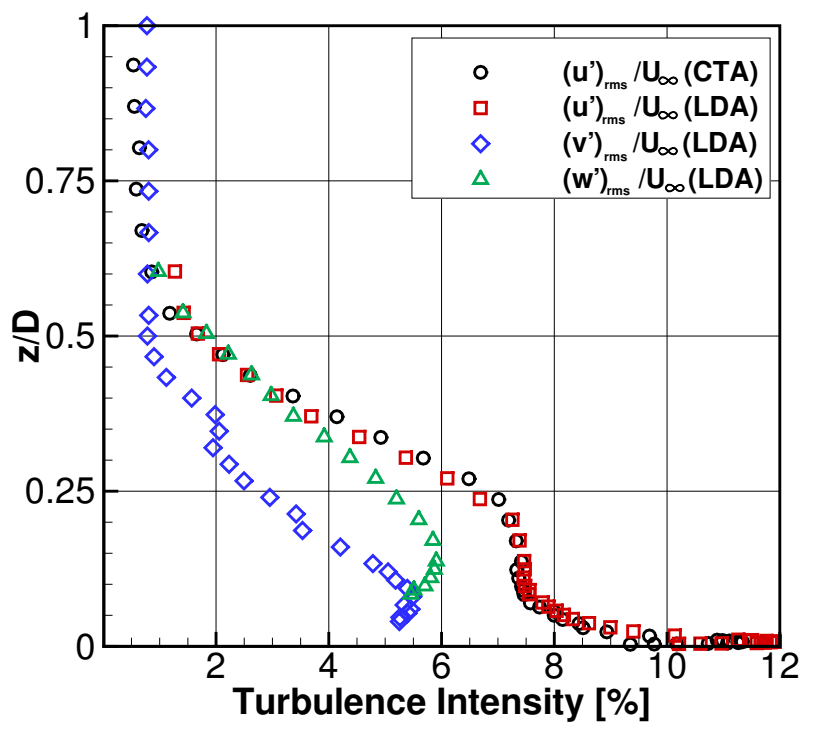

(c) $\left(u^{\prime}\right)_{r m s} / U_{\infty},\left(v^{\prime}\right)_{r m s} / U_{\infty}$ and $\left(w^{\prime}\right)_{r m s} / U_{\infty}$.

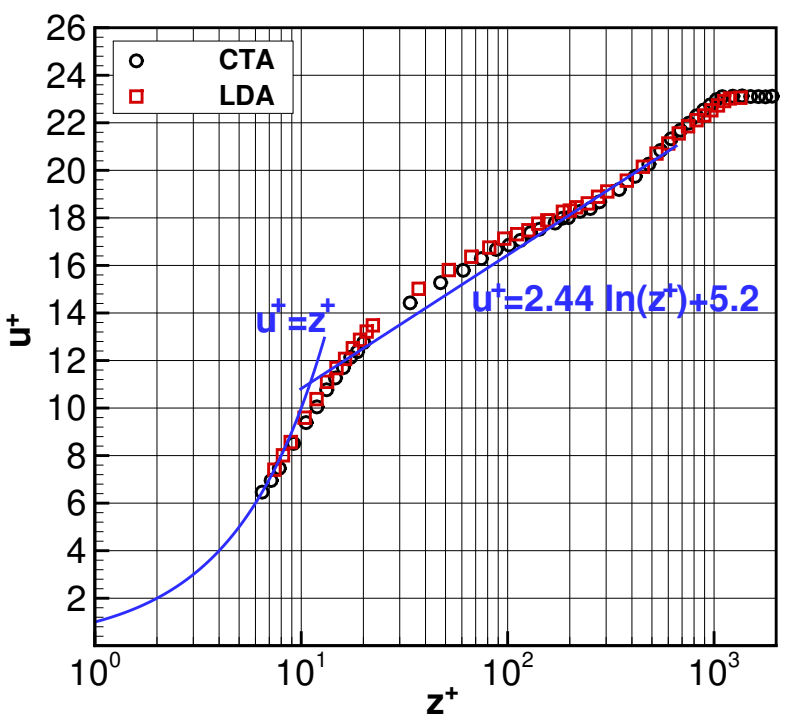

(b) $z^{+}-u^{+}$distribution.

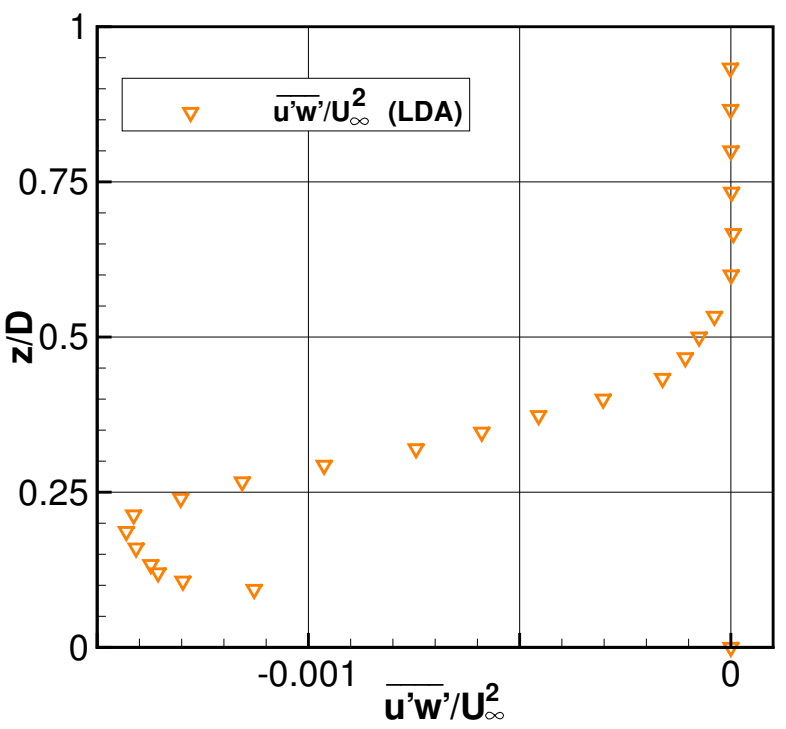

(d) $\overline{u^{\prime} w^{\prime}} / U_{\infty}^{2}$.

Figure 7: Inflow properties of the turbulent boundary layer at the inlet of the test section.

number of $\operatorname{Re}_{\delta_{2}}=2500$. The streamwise turbulent fluctuations $\left(u^{\prime}\right)_{r m s} / U_{\infty}$ gradually increase from the free-stream $(1.2 \%)$ to the near-wall region with a peak value of about $12.1 \%$ at $z / D=0.01$. Contrary to the case of an empty test section without any artificial boundary layer thickening techniques presented in Section 3.1, the free-stream streamwise turbulence intensity for the generated thick boundary layer (TBL) is significantly higher, i.e., $\mathrm{Tu}_{u}^{\mathrm{TBL}}=$ $\left(u^{\prime}\right)_{r m s} / U_{\infty}=1.2 \%$. Consequently, the total free-stream turbulence level also increases to $\mathrm{Tu}_{\text {tot }}^{\mathrm{TBL}}=\sqrt{\frac{1}{3}\left(\overline{u^{\prime 2}}+\overline{v^{\prime 2}}+\overline{w^{\prime 2}}\right)} / U_{\infty} \approx 0.4 \%$.

The Reynolds shear stresses are also useful to describe the flow physics, but complicated to measure accurately. Indeed, in LDA measurements the data collection of each flow component is recorded independently. This means that there is no direct correlation between the two components in a series of measurements due to the independent time signatures in which the droplets pass the specific measuring plane of each component. However, it is still possible to approximate the cross-correlations by utilizing a coincidence algorithm which matches the 
Flow, Turbulence and Combustion, vol. 97 (1), pp. 79-119, (2016).

velocity components by using a window function. This function sets a time interval in which the velocity components are considered to be correlated. In the present case this time interval is set to $6 \mathrm{~ms}$. For sufficient calculation accuracy it is necessary to maintain comparable data rates for each component. For the current setup only the Reynolds shear stress $\overline{u^{\prime} w^{\prime}} / U_{\infty}^{2}$ is of interest for the flow physics. The other cross-components theoretically vanish due to the homogeneity of the spanwise direction. Fig. $7(\mathrm{~d})$ presents the distribution of $\overline{u^{\prime} w^{\prime}} / U_{\infty}^{2}$ at $x / D=-1.5$ in the symmetry plane. As expected for a positive mean velocity gradient at the wall, the Reynolds shear stress is negative.

In conclusion, the predefined inlet conditions of a fully developed turbulent boundary layer with a desired thickness of $\delta / D \approx 0.5$ have been achieved by using customized vortex generators in the nozzle of the wind tunnel. The obtained data are verified by comparison with the $1 / 7$ power law as the reference for the velocity distribution. Furthermore, an overall reasonable distribution of the turbulent fluctuations similar to a natural boundary layer commonly presented in the literature [36-39] is achieved.

\section{Numerical Setup}

\subsection{CFD solver: FASTEST-3D}

To predict the turbulent flow around the hemisphere based on the large-eddy simulation technique, the three-dimensional finite-volume fluid solver FASTEST-3D is used. This in-house code is an enhanced version of the original one $[40,41]$. To solve the filtered Navier-Stokes equations for LES, the solver relies on a predictor-corrector scheme (projection method) of second-order accuracy in space and time [42]. The discretization relies on a curvilinear, blockstructured body-fitted grid with a collocated variable arrangement. The surface and volume integrals are calculated based on the midpoint rule. Most flow variables are linearly interpolated to the cell faces leading to a second-order accurate central scheme. The convective fluxes are approximated by the technique of flux blending $[43,44]$ to stabilize the simulation. For the current case the flux blending includes $5 \%$ of a first-order accurate upwind scheme and $95 \%$ of a second-order accurate central scheme. A preliminary study shows that these settings are a good compromise between accuracy and stability. The momentum interpolation technique of Rhie and Chow [45] is applied to couple the pressure and the velocity fields on non-staggered grids.

Since LES is used, the large scales of the turbulent flow field are resolved directly, whereas the non-resolvable small scales have to be taken into account by a subgrid-scale (SGS) model. Different SGS models based on the eddy-viscosity concept are available in FASTEST-3D: The well-known and most often used Smagorinsky model [46], the dynamic Smagorinsky model according to Germano et al. [29] and Lilly [47], and the WALE model [28]. Owing to the moderate Reynolds number considered and the fine grid applied, the SGS model is expected to have a limited influence on the results. Nevertheless, in order to investigate and verify this issue, simulations of the flow around the hemisphere are carried out applying the above mentioned SGS models. For this purpose, a constant inflow velocity profile (1/7 power law) without any turbulent fluctuations is assumed. The results are analyzed in Appendix Appendix B. This SGS investigation shows that the Smagorinsky model with $0.065 \leq C_{s} \leq 0.1$ or the dynamic Smagorinsky model basically leads to the same results. The WALE model with $C_{W}=0.33$ (value corresponding to the classical Smagorinsky model with $C_{s}=0.1$ [28]) produces a nearly identical flow except for the region upstream to the hemisphere. Therefore, as the best compromise between accurate results and fast computations, the standard Smagorinsky model with the constant set to $C_{s}=0.1$ is used for the present case. 
Flow, Turbulence and Combustion, vol. 97 (1), pp. 79-119, (2016).

FASTEST-3D is efficiently parallelized based on the domain decomposition technique relying on the Message-Passing-Interface (MPI). Non-blocking MPI communications are used and offer a non negligible speed-up compared to blocking MPI communications [48].

\subsection{Computational domain}

To simulate the problem using a block-structured mesh, different forms of the computational domain with according boundary conditions were tested during preliminary tests. Finally, the chosen computational domain is the simplest one: A large hemispherical expansion with its origin at the center of the hemisphere (see Fig. 8(a)). This domain is originally divided into 5 geometrical blocks, so that nearly orthogonal angles are obtained on the surface of the hemisphere (see Fig. 8(b)) and in the entire volume. To prescribe the inlet and outlet boundary conditions described in the next section, the upper, left and right blocks are divided along the $x / D=0$ plane leading to 8 geometrical blocks (see Fig. 8(a)). The distribution of the blocks and the angles on the surface of the hemisphere can be observed in the close-up depicted in Fig. 8(b). Figure 8(c) shows the $x-y$ cross-section of the grid at the bottom wall and Fig. 8(d) depicts the $x-z$ cross-section in the symmetry plane. For the sake of visualization only every fourth grid line of the mesh is shown. Accordingly, the angles between the grid lines and the transitions between the blocks appear to be worse than they are in the full mesh. The 8 geometrical blocks are later split into 80 parallel blocks for the distribution of the computation on a parallel computer. The outer domain has a radius of 10 D. 240 grid points are distributed non-equidistantly based on a geometrical stretching in the expansion direction. 640 points are used at the circumference of the bottom of the hemisphere. The final grid contains $30.72 \times 10^{6}$ control volumes $(\mathrm{CVs})$. In order to fully resolve the viscous sublayer, the first cell center is located at a distance of $\Delta z / D \approx 5 \times 10^{-5}$ from the wall, which leads to averaged $z^{+}$values below 0.25 (see Figs. 8(e) and (f)) and more than 50 points in the boundary layer on the hemisphere upstream to the separation. The geometrical stretching ratios are kept below 1.05. The aspect ratio of the cells on the hemispherical body are low, i.e., in the range between 1 and 10. This yields a dimensionless cell size in the two tangential directions below 29, which fits to the recommendation of Piomelli and Chasnov [49] for a wall-resolved LES. Note that the resolution of the grid is chosen based on extensive preliminary tests not presented here. For this fine grid a small time step of $\Delta t^{*}=\Delta t U_{\infty} / D=3.084 \times 10^{-5}$ is required ensuring a CFL-number below unity.

\subsection{Boundary conditions}

Figure 9 depicts the boundary conditions used in the simulation in colors: Black for the walls, blue for the inlet and red for the outlet. At the bottom of the domain and on the hemisphere a no-slip wall condition is applied justified by the fine near-wall resolution mentioned above. A $1 / 7$ power law with $\delta / D=0.5$ and without any perturbation is applied as inlet condition on the external surface of the domain for $x \leq 0$. Moreover, this power law is applied for all CVs with $x / D \leq-2$ (see the area with hatched lines on Fig. 9). This region $(x / D \leq-2)$ does not need to be solved for the problem. However, it could not be simply cut from the mesh because of the hemispherical form of the block-structured grid. Therefore, for all CVs with $x / D \leq-2$ the flow field is not predicted, so that the mean velocity profile at $x / D=-2$ remains constant in time and perfectly fits the experiment. In order to approximate the turbulent boundary layer depicted in Fig. 7 perturbations produced by a turbulence inflow generator (described in Section 4.4) are injected in a $2 \mathrm{D} \times \mathrm{D}$ window at $x / D=-1.5$ (see Fig. $9(\mathrm{~b})$ ). A zero velocity gradient boundary condition is defined for the outlet on the external surface of the domain for the geometrical blocks 5,6 and 7 as defined in Fig. 8(a)). At the outlet of block 8 where the 
Flow, Turbulence and Combustion, vol. 97 (1), pp. 79-119, (2016).

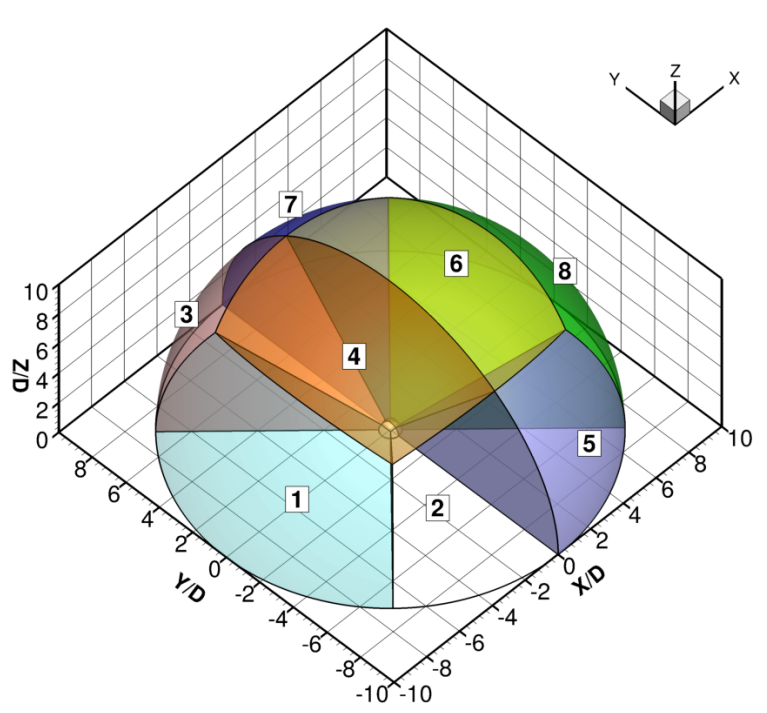

(a) Grid based on a 8 blocks approach.

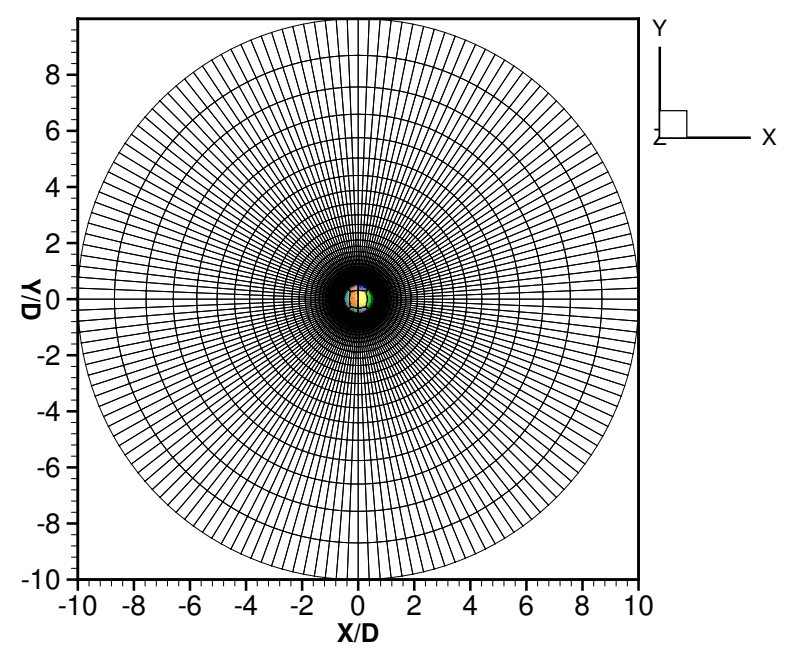

(c) Grid of the $x-y$-plane located at $z=0$ (Note that only every fourth grid line in each direction is displayed here)

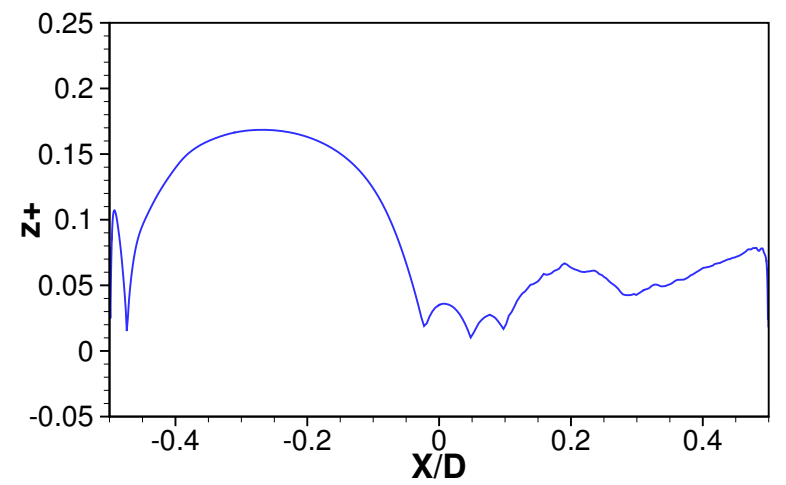

(e) $z^{+}$in the symmetry plane on the hemisphere

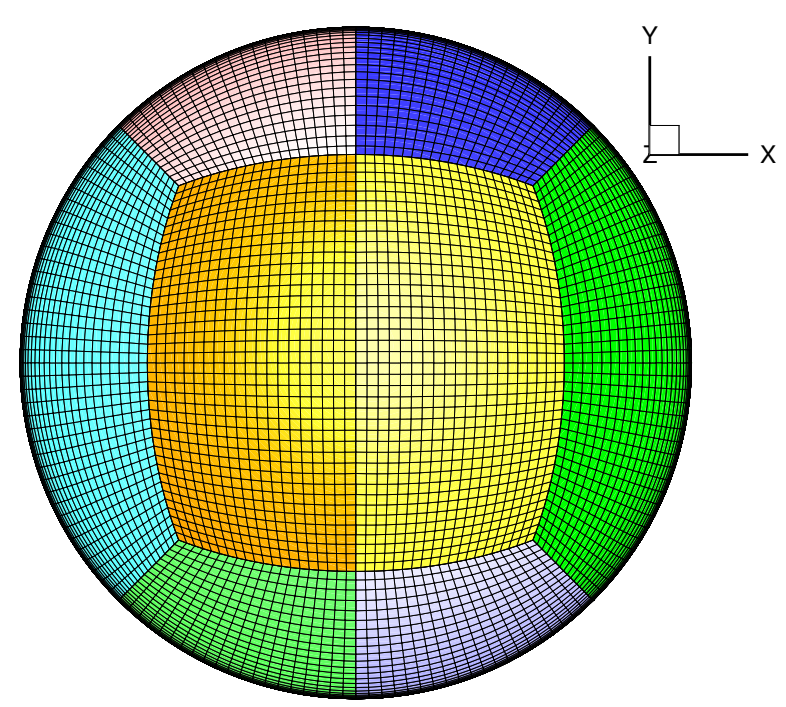

(b) Close-up of the hemispherical surface (Note that only every fourth grid line in each direction is displayed here).
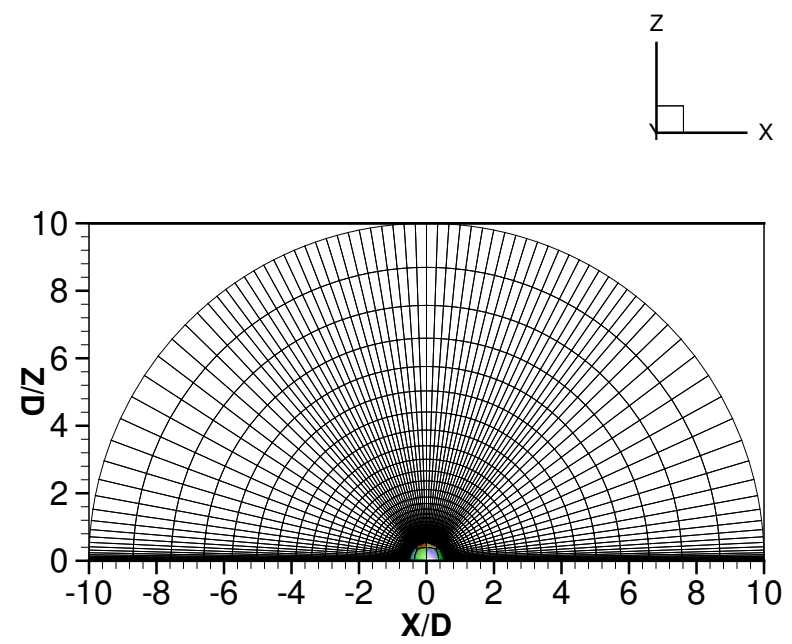

(d) Grid of the $x-z$-plane located at $y=0$ (Note that only every fourth grid line in each direction is displayed here)

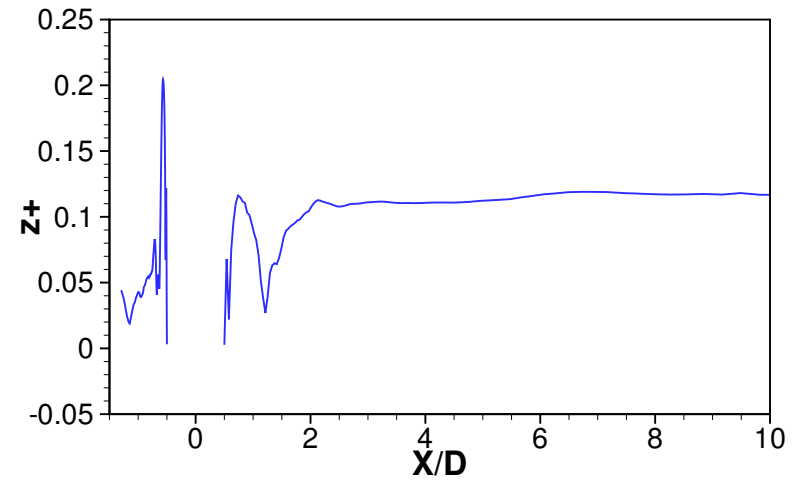

(f) $z^{+}$in the symmetry plane upstream and downstream of the hemisphere

Figure 8: Grid used for the LES prediction of the flow past the hemisphere. 
Flow, Turbulence and Combustion, vol. 97 (1), pp. 79-119, (2016).

large-scale flow structures leave the computational domain, a convective boundary condition is applied with a convective velocity set according to the $1 / 7$ power law. The fact that the simulation does not use symmetry boundary conditions or slip walls at the top or at the lateral sides, is in agreement with the free flow situation in the experiments. Indeed, the test section is open on the top and on the lateral sides.

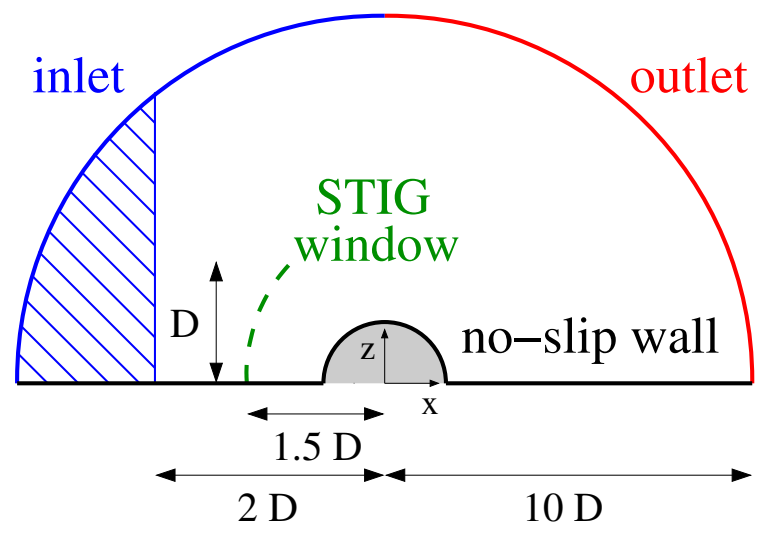

(a) 2D sketch.

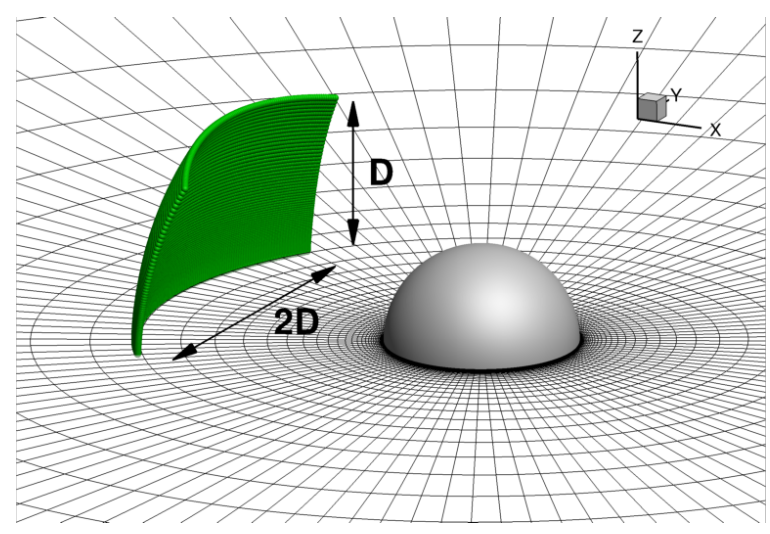

(b) STIG injection window (Note that only every fifth line of the bottom grid is displayed here).

Figure 9: Boundary conditions used for the simulation.

\subsection{Synthetic turbulence inflow generator}

It is commonly known that discrepancies between experimental measurements and results of numerical simulations are often the result of an inadequate representation of the boundary conditions. For LES the absence of an appropriate turbulence intensity at the inlet is a very critical issue (see Appendix Appendix C). In order to circumvent this problem, it is meaningful to incorporate perturbations produced by a turbulence inflow generator into the flow. In the present study the digital filter concept of Klein et al. [50] is applied for this purpose. This method delivers instantaneous three-dimensional velocity distributions matching desired mean velocities and Reynolds stresses with the help of the decomposition of Lund et al. [51]. An integral time scale and two integral length scales are also required to ensure the temporal and spatial coherence of the generated turbulent structures. Details and the successful application of this method for demanding test cases such as the periodic hill flow are shown in Schmidt and Breuer [52].

Regarding the relatively coarse grid resolution at the inflow plane, the usage of the synthetic turbulence inflow generator (STIG) within the LES is not meaningful due to the subsequent damping of the initial velocity fluctuations in this area. Accordingly, a shift of the region where the artificial perturbations are taken into account towards a finer resolved area is a promising approach. Therefore, instead of the application of the STIG at the inflow plane, the velocity fluctuations are imposed inside the computational domain and applied as source terms within the momentum equations. This methodology of imposing the inflow conditions was recently validated based on the flow past a SD7003 airfoil [53]. The position of the STIG window in the present case is at $x / D=-1.5$ with a width of $2 \mathrm{D}$ and a height of $\mathrm{D}$ (see Fig. 9). In order to avoid a discontinuity in the momentum equations, these source terms are not superimposed at a plane. The application region is spatially spread in the streamwise direction, where the amplitude of the source terms follows a Gaussian distribution with the maximum located at $x / D=-1.5$. In the present case the streamwise extension covers 5 cells. 
Flow, Turbulence and Combustion, vol. 97 (1), pp. 79-119, (2016).

Due to the modeling of the boundary layer by the $1 / 7$ power law it is possible to assume the mixing length model by Prandtl to determine an appropriate integral length scale for the STIG. To obtain turbulent structures larger than the biggest cells of the STIG window, the spatial length scale in wall-normal direction is computed at $z^{+}=100$ and leads to $L / D=2.06 \times 10^{-2}$. For the spanwise length scale the same value is chosen. The required integral time scale is based on the Taylor hypothesis leading to $T^{*}=L U_{\infty} /\left(D u\left(z^{+}=100\right)\right)=2.79 \times 10^{-2}$. Based on the grid resolution of the STIG window $\left(-1 \leq y / D \leq 1\right.$ with $\Delta y / D=9.713 \times 10^{-3}$ and $0 \leq z / D \leq 1$ with $\Delta z / D=2.191 \times 10^{-3}$ ) and the employed time step of the simulation $\Delta t^{*}=\Delta t U_{\infty} / D=3.084 \times 10^{-5}$, the normalized scales and the support of the filters as defined in Klein et al. [50] are $n_{\mathrm{t}} \times n_{\mathrm{y}} \times n_{\mathrm{z}}=906 \times 2 \times 9$ and $N_{\mathrm{t}} \times N_{\mathrm{y}} \times N_{\mathrm{z}}=1812 \times 4 \times 18$, respectively. The experimental velocity distribution and the measured normal and shear components of the Reynolds stress tensor presented in Section 3.3 are used as input values for the STIG. The missing parts of the Reynolds stress components close to the wall are filled up with the help of the DNS data by Schlatter et al. [39] (see Fig. 10). The $v$ - and $w$-components of the mean velocity are set to zero as well as the Reynolds stress components $\overline{u^{\prime} v^{\prime}} / U_{\infty}^{2}$ and $\overline{v^{\prime} w^{\prime}} / U_{\infty}^{2}$

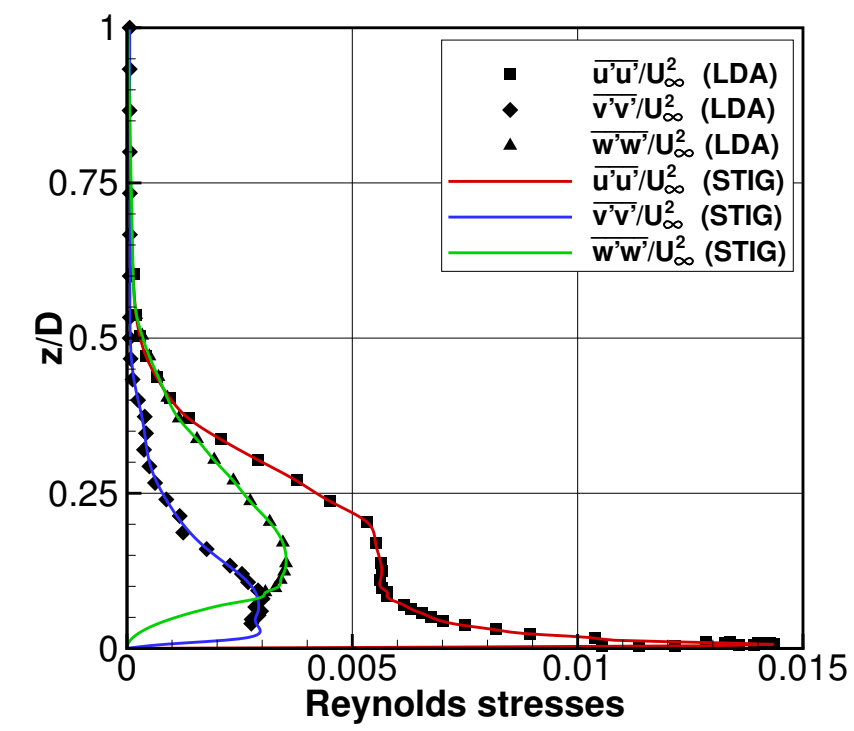

(a) Normal Reynolds stresses.

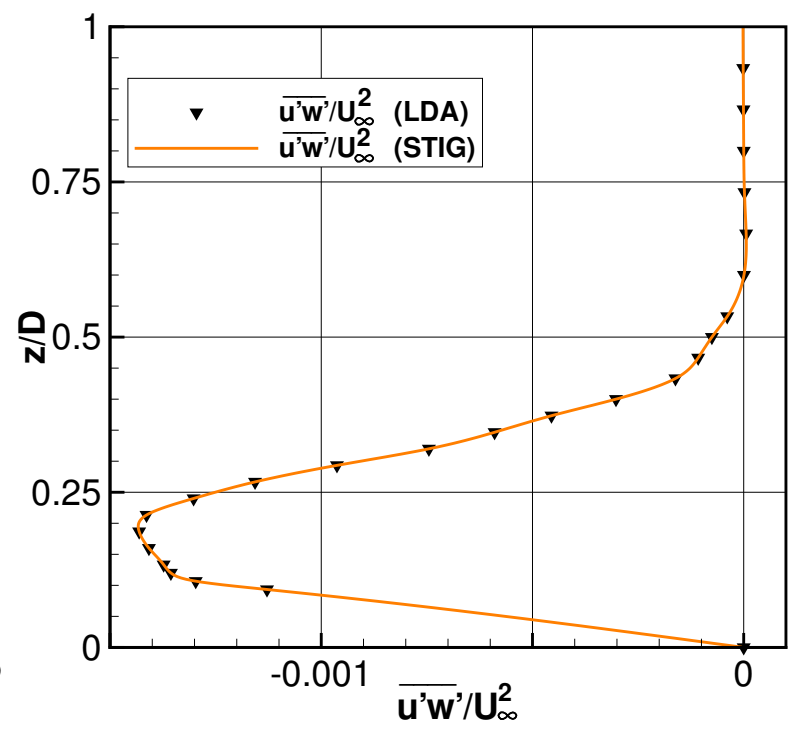

(b) Shear Reynolds stress $\overline{u^{\prime} w^{\prime}} / U_{\infty}^{2}$.

Figure 10: Distributions of the Reynolds stresses derived from the experiment as input parameters for the application of the synthetic turbulence inflow generator.

160,000 time steps are generated to provide a sufficiently long time series of inflow data. Figure 11 exemplarily shows the instantaneous streamwise velocity distribution at one arbitrarily chosen time step.

\section{Unsteady Results}

This section provides an overview of the main physical flow characteristics observed in the present study. The first systematic classification map of the unsteady flow patterns past a hemisphere was presented by Savory and Toy [7] comprising several clearly distinguishable regions. A visualization of these regions is depicted in Fig. 12 which shows the results of the instantaneous flow field at an arbitrary instant in time. Figure 12(a) presents the unsteady velocity field of the large-eddy simulation based on the main flow component $\bar{u} / U_{\infty}$ in the symmetry $x-z-$ plane of the hemisphere. An attempt to capture the unsteady flow features in 


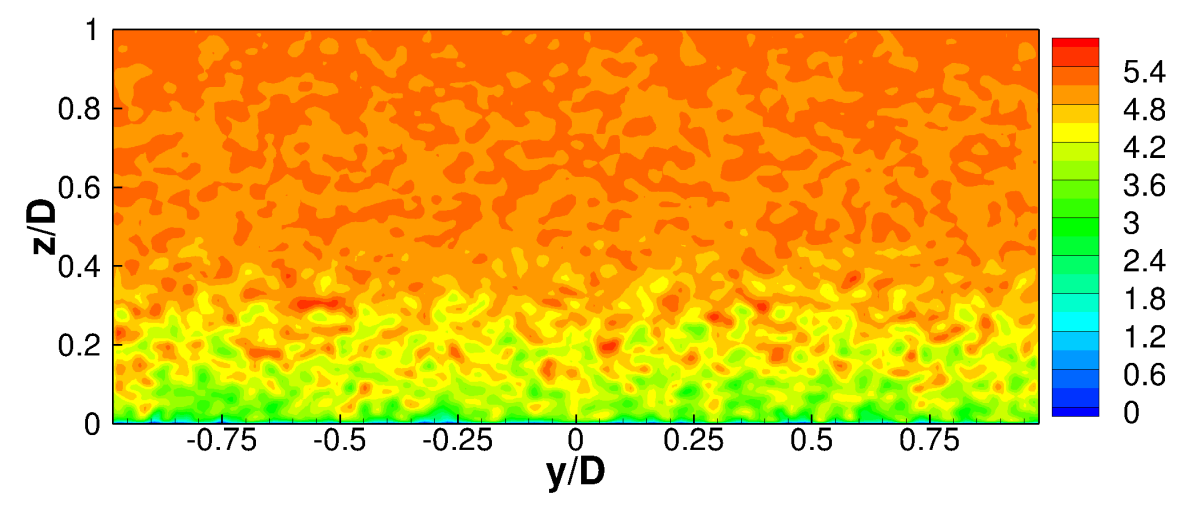

Figure 11: Example of the instantaneous streamwise velocity distribution generated for the given STIG window.

the experiment is shown in Fig. 12(b). The model of the hemisphere is illuminated by a strong laser light-sheet focused on the symmetry plane. The flow was seeded with DEHS droplets as used for the laser-Doppler measurements. In the following a detailed look at the highlighted spots $1-7$ of both pictures shall help to expose the specific flow regions initially determined by Savory and Toy [7].

The upstream flow field is characterized by the development of a horseshoe vortex system (1) which results from the separation of the boundary layer from the ground. This effect is mainly driven by the positive pressure gradient in front of the hemisphere which acts as a flow barrier. The size and formation of this particular flow structure also depends on the properties of the approaching boundary layer such as the turbulence intensity, the velocity distribution and the overall thickness of the boundary layer. The stagnation area (2) of the flow is located close to the lower front surface of the hemisphere, where the stagnation point is found at the surface at an angle of about $\theta_{\mathrm{stag}}^{\mathrm{LDA}} \approx 166^{\circ}$ (definition of $\theta$ in Fig. 12(a)). Behind this region the flow is accelerated (3) which leads to the generation of strong near-wall vorticity. The flow detaches from the surface of the hemisphere along a separation line (4) at a separation angle of $\theta_{\text {sep }}^{\mathrm{LDA}} \approx 90^{\circ}$. The wake of the hemisphere is divided into the recirculation area and the outer flow field (5). Strong shear layer vorticity (6) can be observed leading to the production of KelvinHelmholtz vortices which travel downstream in the flow field. The size of the recirculation area behind the hemisphere can be evaluated by the reattachment point (7) of the flow in the symmetry plane. This last region is not clearly visible in the experiment and therefore not shown in Fig. 12(b). In the reattachment area the "splatting" effect occurs, redistributing momentum from the wall-normal direction to the streamwise and spanwise directions.

A complementary view of the instantaneous flow illustrating the 3D-vortex distribution around the hemisphere is presented in Fig. 13. The flow structures are visualized using iso-surfaces of the pressure fluctuations $\left(p^{\prime} /\left(\rho_{\text {air }} U_{\infty}^{2}\right)=-2.47 \times 10^{-4}\right)$ as recommended by García-Villalba et al. [24]. The upstream near-wall flow is dominated by the horseshoe vortex system that trails past the hemisphere and forms stable "necklace"-vortices that stretch out into the wake region. The flow detaches from the surface of the hemisphere along the indicated separation line and the vortices "roll-up". The shedding type and frequency are varying in time along the separation line as described below. Moreover, these rolled-up vortices interact with the horseshoe vortices just after the hemisphere and in some cases they merge generating bigger vortical structures. The results are entangled vortical "hairpin"-structures of different sizes and orientations traveling downstream and forming a "vortex chain". Schematic 3D sketches and explanations of the formation of these flow structures around and behind the hemisphere can be found in the literature $[11,12]$. Note that smaller "hairpin"-structures can be also 


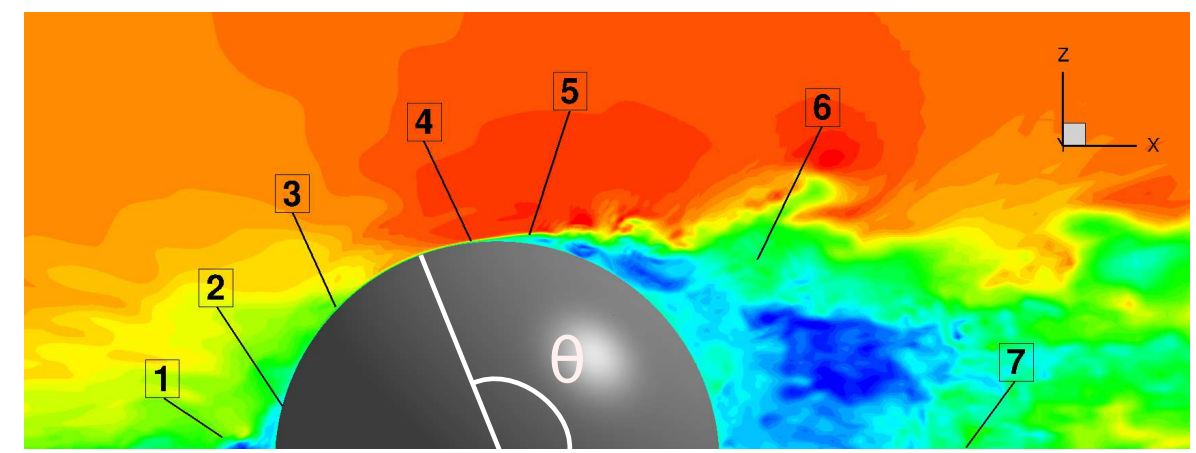

(a) Characteristic regions of the flow past a hemisphere as classified by Savory and Toy [7].

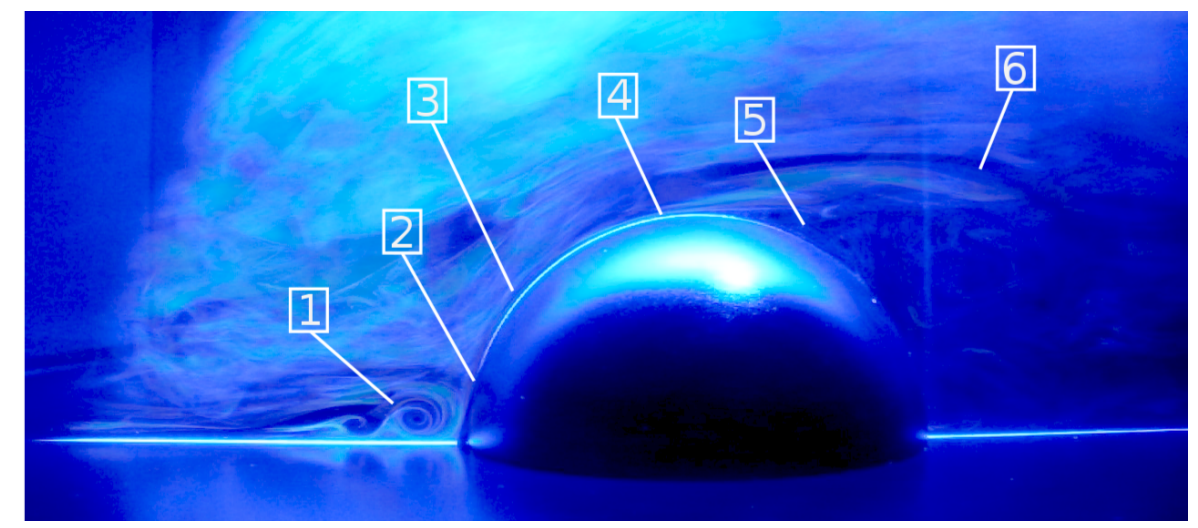

(b) Flow visualization of the experiment.

Figure 12: Visualization of flow regions and characteristic flow features of the flow past the hemisphere: (1) horseshoe vortex system, (2) stagnation area, (3) acceleration of the flow, (4) separation point, (5) dividing streamline, (6) shear layer vorticity, (7) reattachment point.

observed in the wake growing from the ground as usual in a turbulent boundary layer. The shape of the instantaneous vortices traveling downstream are depending on the shedding type. In order to identify the different shedding processes and their frequencies, it is common to plot velocity spectra at specific locations of the flow field. The present study examines the velocity spectra of two monitoring points $P_{1}$ and $P_{2}$ (see Fig. 14) located in the wake of the hemisphere outside of the recirculation area. The position of $P_{1}$ and $P_{2}$ is chosen based on the analysis of Manhart [19]. The positions of the points are visualized in Fig. 14(b) for the side view and in Fig. $14(\mathrm{c})$ for the top view. Monitoring point $P_{1}(x / D=1.25, y / D=$ $0, z / D=0.4)$ is located in the symmetry plane. The monitoring point $P_{2}$ is located outside of the symmetry plane $(x / D=1.25, y / D=0.37, z / D=0.16)$. Indeed, at these two locations the dominant shedding frequencies are clearly visible. In order to be sure to capture all frequencies of the wake flow and to get smooth velocity spectra, the data have to be collected with an adequate sampling rate during a long time period. Therefore, measurements are more appropriate than LES predictions for this purpose. The measurements include a sampling rate of $1 \mathrm{kHz}$ and are collected over a period of 30 minutes with the same hot-film probe as mentioned in Section 3.3. This 1D-probe is restricted to the measurement of the streamwise velocity component $u$.

The power spectral density (PSD) of each location is given in Fig. 14(a) where the colors of the lines refer to the particular colors of the monitoring point, red and blue, respectively. At $P_{1}$ the PSD is high between $7.9 \mathrm{~Hz} \leq f_{1} \leq 10.6 \mathrm{~Hz}\left(0.23 \leq \mathrm{St}_{1}=f_{1} D / U_{\infty} \leq 0.31\right)$. A maximum is reached at about $f_{1}=9.2 \mathrm{~Hz}\left(\mathrm{St}_{1} \approx 0.27\right)$. At $P_{2}$ outside the symmetry plane a 

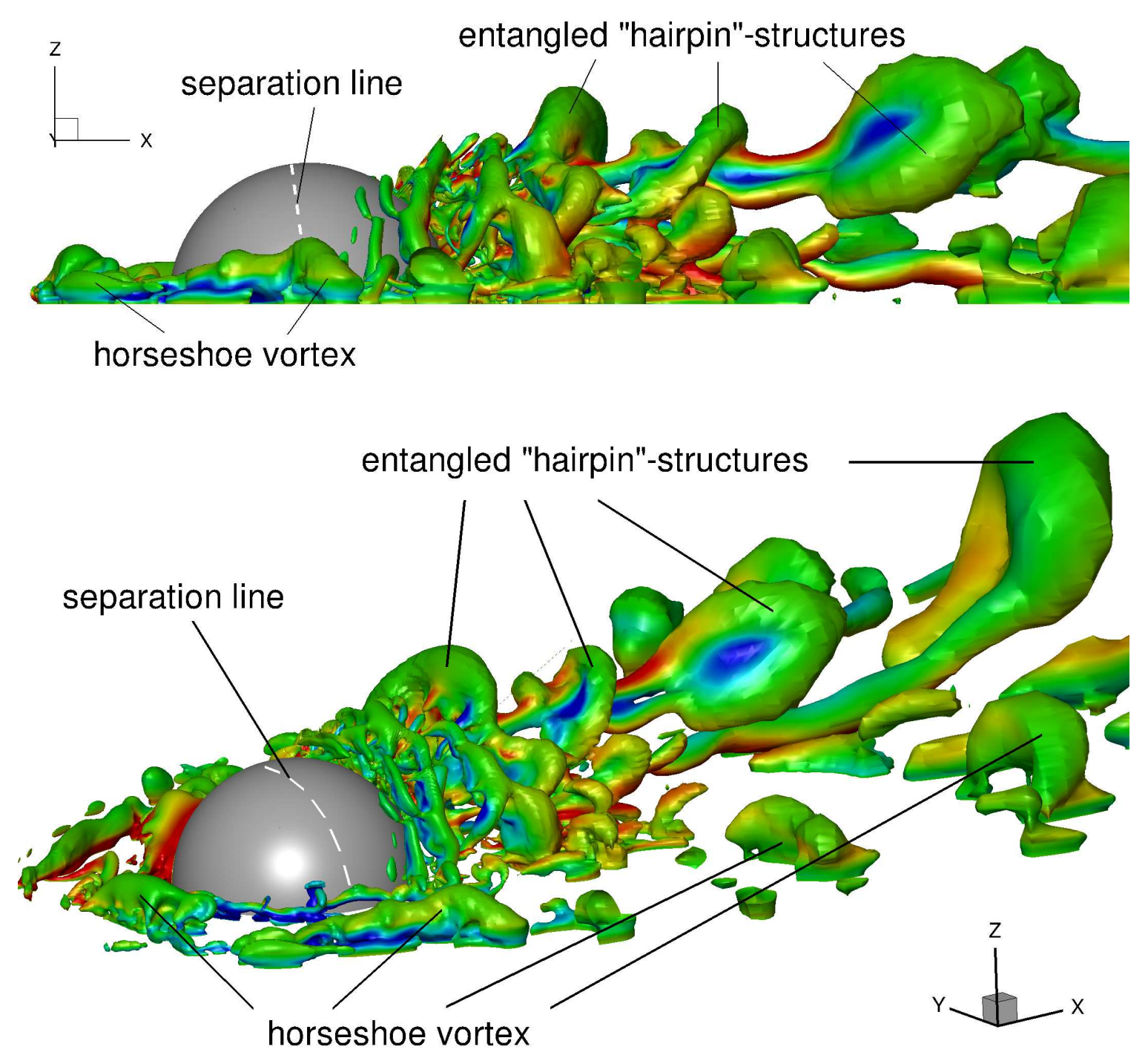

Figure 13: Snapshot of unsteady vortical structures visualized by utilizing the iso-surfaces of the pressure fluctuations $\left(p^{\prime} /\left(\rho_{\text {air }} U_{\infty}^{2}\right)=-2.47 \times 10^{-4}\right)$ colored by the spanwise instantaneous velocity.

clearly detectable frequency peak is found at $f_{2}=5.5 \mathrm{~Hz}$ corresponding to a Strouhal number of $\mathrm{St}_{2} \approx 0.16$. This suggests the presence of two vortex shedding types in the wake: The separation of the boundary layer at the top of the hemisphere leads to a detachment of "archtype"-vortices observed at the monitoring point $P_{1}$ with a shedding frequency in the range $7.9 \mathrm{~Hz} \leq f_{1} \leq 10.6 \mathrm{~Hz}$. This phenomenon can be visualized based on the LES data (see Fig. 15). The second type is a "von Kármán"-shedding process occurring at a shedding frequency of $f_{2}=5.5 \mathrm{~Hz}$ on the sides of the hemisphere captured at point $P_{2}$.

This second shedding process involves an interesting pattern of two clearly distinguishable types that switch in shape and time (see Fig. 16). The first kind can be described as a "quasisymmetric" process where the vortical structures form and detach in a "double-sided" symmetric manner (visualized by the velocity magnitude near the wall in Fig. 16(a) and schematically depicted in Fig. 16(c)) leading to "arch-type"-vortices [54] or "symmetric"-vortices [55]). The second kind relates to a "quasi-periodic" vortex shedding resulting in a "single-sided" alternating detachment pattern (visualized by the velocity magnitude near the wall in Fig. 16(b) and schematically depicted in Fig. 16(d)), classically observed in the wake of flow fields past geometrically two-dimensional obstacles such as the cylinder flow. Both patterns substitute 


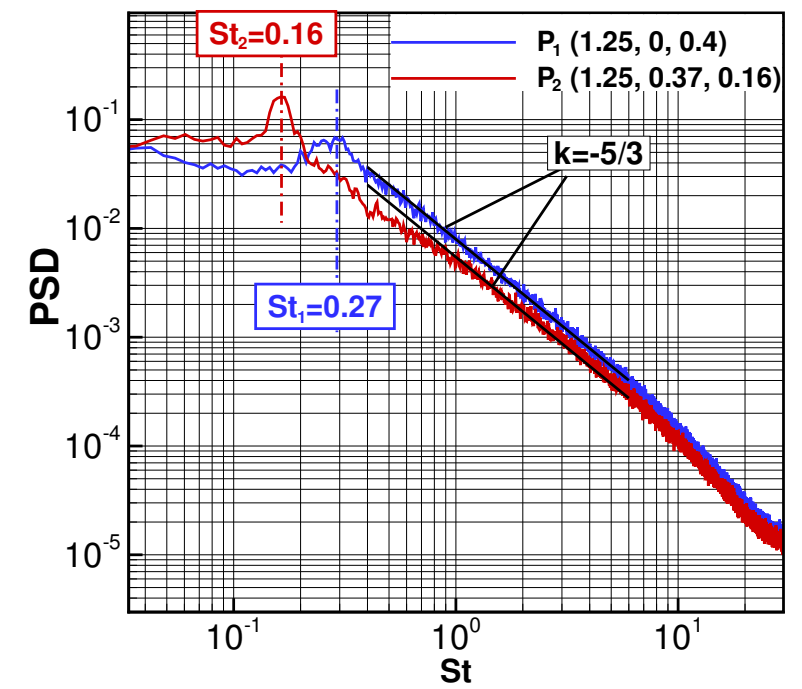

(a) PSD with dominant Strouhal numbers in the wake.

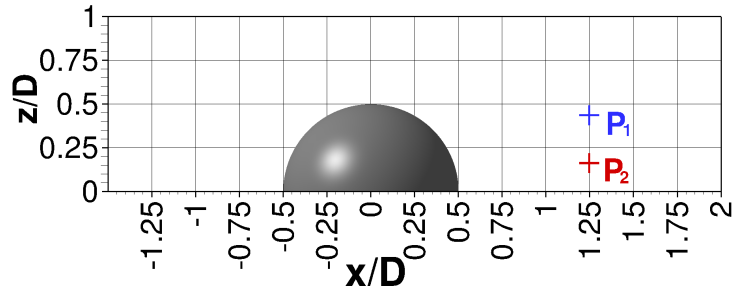

(b) Location of points in $x-z$-plane.

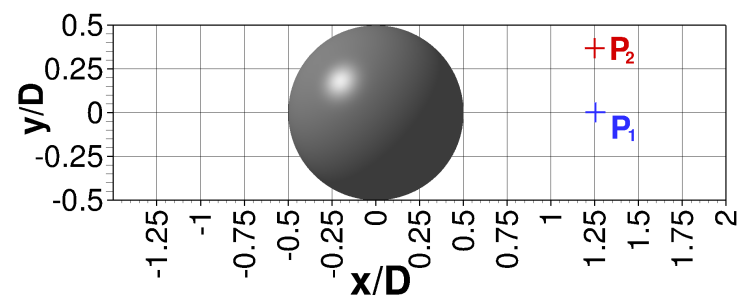

(c) Location of points in $x-y$-plane.

Figure 14: Velocity spectra at the monitoring points $P_{1}$ and $P_{2}$ in the wake regime of the hemisphere.

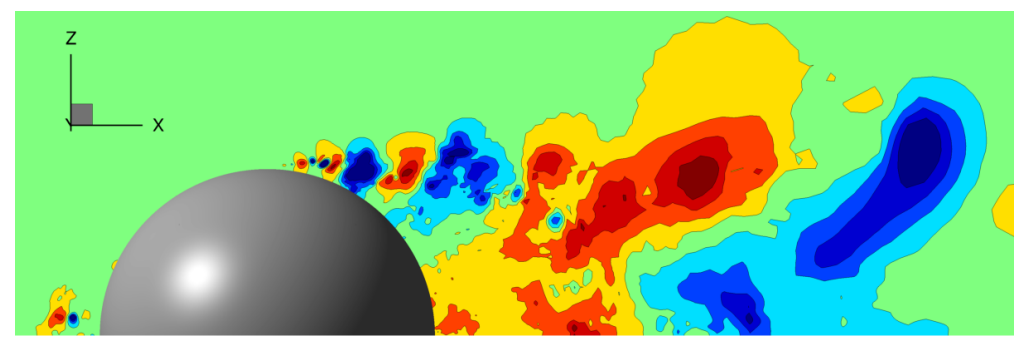

Figure 15: Vortex shedding from the top of the hemisphere visualized by the pressure fluctuations of the LES in the symmetry plane.

themselves in time so that either the symmetric or the alternating shedding type is observable in the wake flow during a certain period of time. Manhart [19] observes this behavior, too. He assumed that the symmetric shedding type is mainly driven by small-scale, less energetic turbulent structures in the flow field. It nearly completely vanished in his predictions when performing a large-eddy simulation on a rather coarse grid, where the small-scale flow structures cannot be resolved appropriately.

It has to be noted that the large-eddy simulations of Manhart [19] cannot be compared directly with the present case due to the differences in the Reynolds number and the artificial surface model roughness. Nevertheless, the qualitative results suggest a similar behavior for the two major shedding frequencies.

Unsteady flow results are useful to get an impression of the overall complexity of the observed flow phenomena. For a general characterization, statistical evaluations of the flow field are more appropriate. Therefore, the next paragraph presents the time-averaged flow field.

\section{Time-averaged Results and Discussion}

This section presents the time-averaged results of the flow around the hemisphere. The instantaneous flow was averaged over a long time period of about 1370 dimensionless time units ${ }^{4}$ for

\footnotetext{
${ }^{4}$ Time normalized by $U_{\infty}$ and $D$.
} 


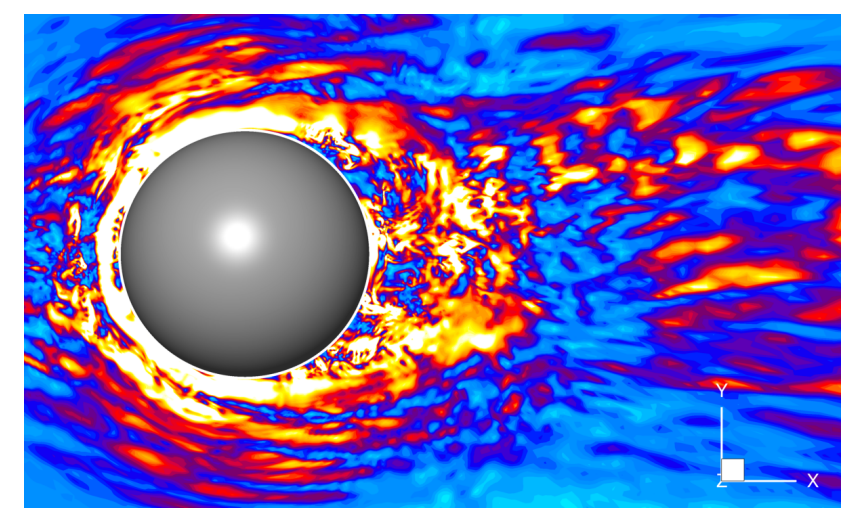

(a) Symmetric shedding type (velocity magnitude near the bottom wall).

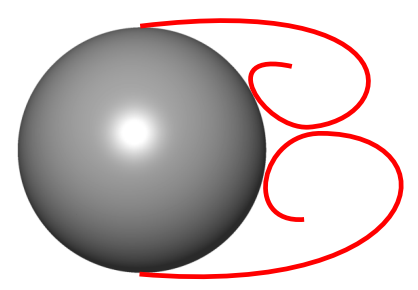

(c) Schematic of the symmetric shedding type.

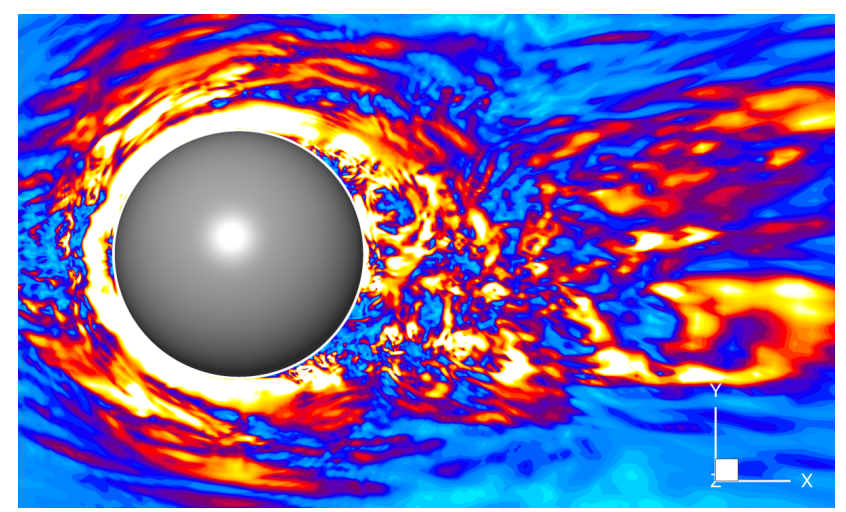

(b) Alternating shedding type (velocity magnitude near the bottom wall).

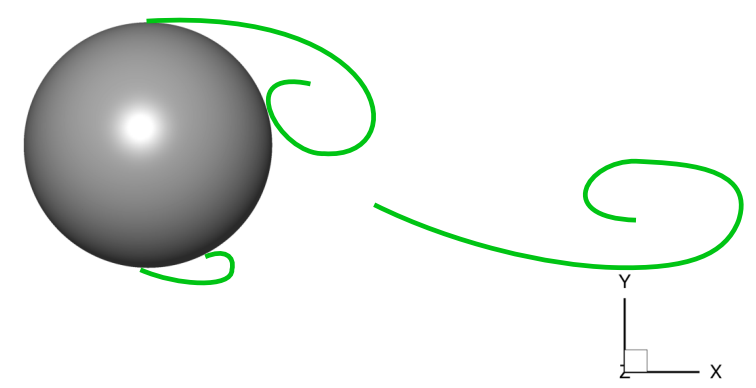

(d) Schematic of the alternating shedding type.

Figure 16: Visualization of the two vortex shedding types present in the wake behind the hemisphere.

the LDA measurements which ensures a sufficient amount of data. Due to the small time step, the LES results are averaged over a shorter period of 86 dimensionless time units. This seems to be sufficient relying on the investigations of García-Villalba et al. [24]. The outlined approach using two-dimensional color plots offers the possibility to identify characteristic regions that are introduced and discussed in the previously mentioned publications (see Section 1). The key aspects focus on the detailed view of the velocity field and the associated Reynolds stresses in the symmetry plane and a chosen spanwise plane in the wake. The laser-Doppler measurements are used for the interpretation of the physical characteristics of the flow. In parallel, the results obtained by the large-eddy simulation are compared with the experimental data and offer details in areas where the measurements are limited. Additionally, selected profiles at specific locations along the streamwise direction of the flow field are chosen for a critical quantitative comparison between the experiment and the simulation. Finally, three-dimensional properties specific to the flow are discussed based on the LES results. The discussion begins with the characteristics of the symmetry plane.

\subsection{Symmetry plane}

Based upon the classification map mentioned in Section 5, the flow field in the symmetry plane is analyzed with regard to the characteristic regions stated by Savory and Toy [7].

\subsubsection{Characteristics of the flow field}

Figure 17 depicts the velocity field around the hemisphere focusing on the streamwise and the wall-normal components. The LDA measurements, presented on the left, are compared with the results of the large-eddy simulation, presented on the right. 


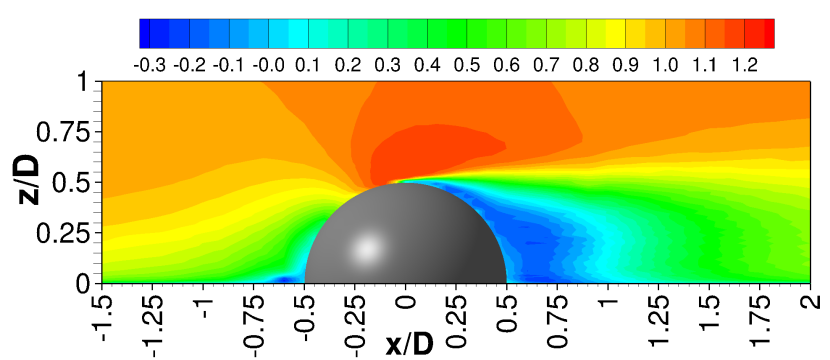

(a) $\bar{u} / U_{\infty}(\mathrm{LDA})$.

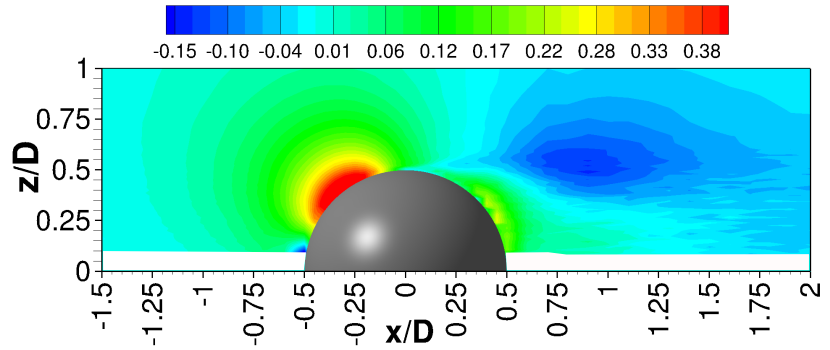

(c) $\bar{w} / U_{\infty}(\mathrm{LDA})$.

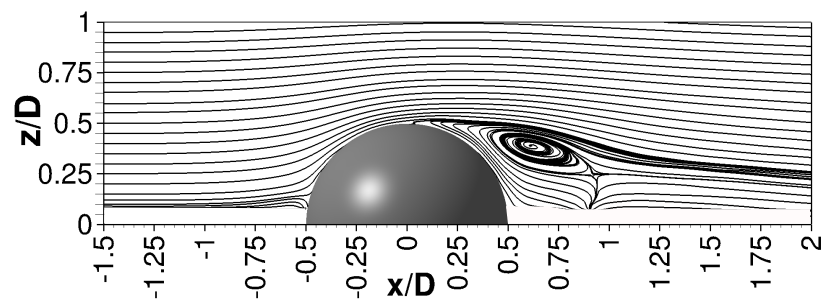

(e) Time-averaged streamlines (LDA).

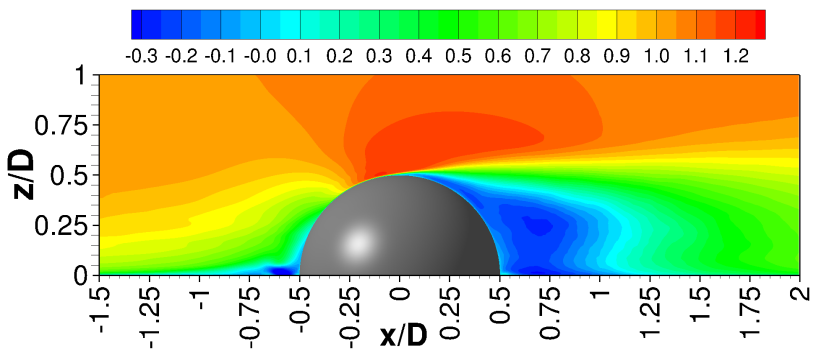

(b) $\bar{u} / U_{\infty}(\mathrm{LES})$.

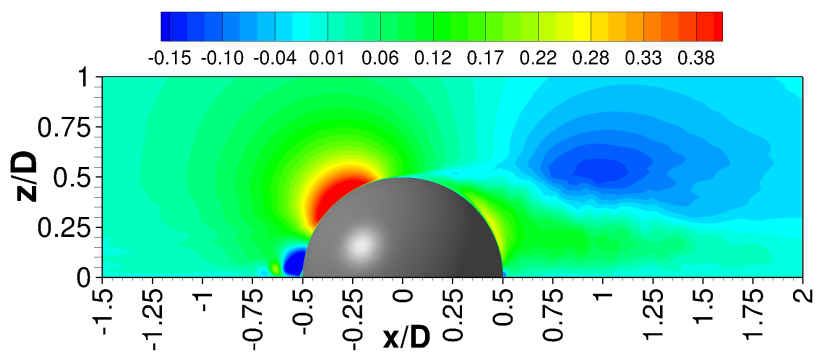

(d) $\bar{w} / U_{\infty}(\mathrm{LES})$.

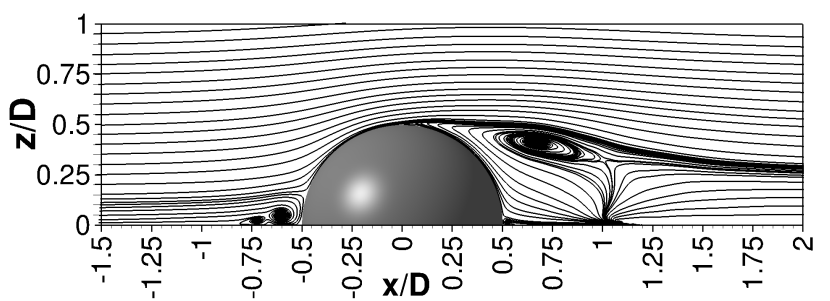

(f) Time-averaged streamlines (LES).

Figure 17: Comparison of the experimental and numerical time-averaged velocity components and streamlines in the symmetry $x-z-$ plane at $y / D=0$.

The streamwise velocity component $\bar{u} / U_{\infty}$ is shown in Figs. 17 (a) and (b). As a fundamental flow characteristic the oncoming flow upstream of the hemisphere in the region $-1.5 \leq x / D \leq$ -0.75 is investigated. The experimental results show that the thickness of the approaching boundary layer is matching the height of the hemisphere well with $z / D \approx 0.5$. A comparable velocity distribution is visible in the large-eddy simulation. The development of a recirculation area can be perceived close to the lower front of the hemisphere between $-0.75 \leq x / D \leq-0.5$. This phenomenon is connected to the horseshoe vortex system. It results from the reorganization of the approaching boundary layer which detaches from the ground at $x_{\text {detach }}^{\mathrm{LES}} / D=-0.97$ due to the positive pressure gradient (stagnation area) located at the bottom front of the hemisphere at about $\theta_{\text {stag }}^{\mathrm{LDA}}=166^{\circ}$ in the measurements and at about $\theta_{\text {stag }}^{\mathrm{LES}}=161^{\circ}$ in the simulation. The size of the horseshoe vortex depends on the turbulence intensity of the approaching flow (see Appendix Appendix $\mathrm{C}$ for the results with and without inflow turbulence). Although the inflow conditions of the synthetic turbulence inflow generator are adjusted to the experimental boundary layer, the horseshoe vortex shows slightly larger expansions in case of the numerical simulation.

The next distinct location is the separation point where the flow detaches from the surface of the hemisphere. It marks an important characteristic for the validation of numerical simulations since its position depends on multiple physical flow properties such as Reynolds number, turbulence intensity of the boundary layer and surface roughness. After exceeding the separation point the flow detaches at an angle of $\theta_{\text {sep }}^{\mathrm{LDA}} \approx 90^{\circ}$ in case of the laser-Doppler measurements. A comparable angle of $\theta_{\text {sep }}^{\mathrm{LES}}=92^{\circ}$ is evaluated for the LES. The separated flow leads to the 
Flow, Turbulence and Combustion, vol. 97 (1), pp. 79-119, (2016).

development of a free shear layer which can be observed as a strong velocity gradient between the outer flow field and the recirculation area in the wake. The size of the recirculation area stretches up to $x / D \approx 1.0$ in the experiment and in the simulation. It is interrelated to the turbulence intensity of the approaching boundary layer. According to previous studies [6, 8, 23, 25] the turbulence level of the oncoming flow influences the length of the recirculation area since with increasing turbulence intensity the location of the separation point is shifted to a further downstream position on the hemisphere. An investigation comparing LES predictions without and with STIG data strongly supports this observation (see Appendix Appendix C). The flow reattaches at about $x_{\text {reattach }}^{\mathrm{LDA}} / D=1.04$ in case of the measurements and at about $x_{\text {reattach }}^{\mathrm{LES}} / D=1.16$ in the simulation.

The wall-normal velocity component $\bar{w} / U_{\infty}$ is presented in Figs. $17(\mathrm{c})$ and (d). The flow field close to the bottom wall is not resolved in the experimental investigation due to the restrictions of the chosen setup (see Section 3.2.1). The missing data is blanked out in white. A notable region is the area of increasing velocity at the front side of the hemisphere at $-0.45 \leq x / D \leq-0.15$ and $0.25 \leq z / D \leq 0.45$ resulting from the acceleration of the fluid after exceeding the stagnation area. The size of this area and the velocity magnitude are almost identical for both LDA measurements and LES. A similar phenomenon can be detected at about $0.5 \leq x / D \leq 1.5$ and $0.40 \leq z / D \leq 0.85$ above the recirculation area. Again the extensions of this region are almost congruent in shape and dimension. A comparison of the streamline plots of the experiment and the numerical simulation are presented Figs. 17(e) and (f). In conclusion, the overall velocity distributions found for the experiment and the numerical simulation are very similar. A closer view using specific velocity profiles at certain positions within the flow field provides a more detailed insight into the quantitative data.

Figure 18 depicts the velocity distribution at specific locations along the symmetry plane for the streamwise (Fig. 18(a)) and the wall-normal (Fig. 18(b)) component. Both figures outline the results of the large-eddy simulation as blue solid lines superimposed by the discrete measuring points of the LDA data represented by black squares. For the sake of clearness only every second measuring point of the experimental results is shown. The chosen distributions in each picture can be subdivided into the upstream region, the hemisphere and the wake region, each consisting of three profiles. Overall, a very good agreement for both the streamwise and the wall-normal component is achieved between LES predictions and LDA measurements. A characteristic position of the flow field in front of the hemisphere is at $x / D=-0.6$. This profile represents the position of the horseshoe vortex system with a strong backflow in the near-wall region that is well predicted by the large-eddy simulation. Another representative position of the flow field is located at $x / D \geq 0.25$. The results show an excellent coincidence concerning the developing shear layer and the velocity distribution in the wake.

\subsubsection{Reynolds stresses}

This section provides a view at the second-order moments in terms of Reynolds stresses. Figures 19(a) and (b) refer to the normal Reynolds stress $\overline{u^{\prime} u^{\prime}} / U_{\infty}^{2}$. The turbulence intensity in the approaching boundary layer is clearly visible in case of the laser-Doppler measurement. In the large-eddy simulation the incoming turbulence intensity is not so strong even through an equal turbulence intensity level is imposed at the STIG window. Indeed, a part of the generated turbulent fluctuations are damped by the numerical discretization: The grid is non-equidistant and the flux blending includes $5 \%$ of a first-order upwind scheme. Despite this difference of the inflow, the shape, location and magnitude of the normal Reynolds stress are well predicted by the large-eddy simulation in the rest of the domain. The highest Reynolds stresses appear in the free shear layer right after the separation point due to the rapid roll-up process of 


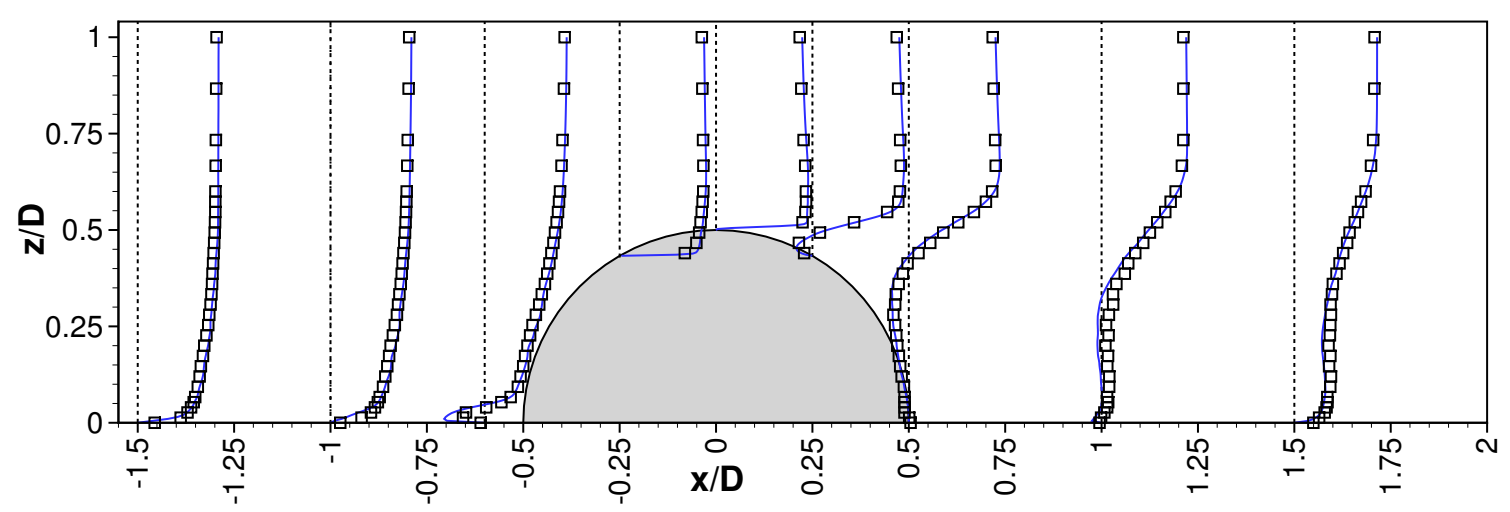

(a) $0.21 \bar{u} / U_{\infty}+x / D$.

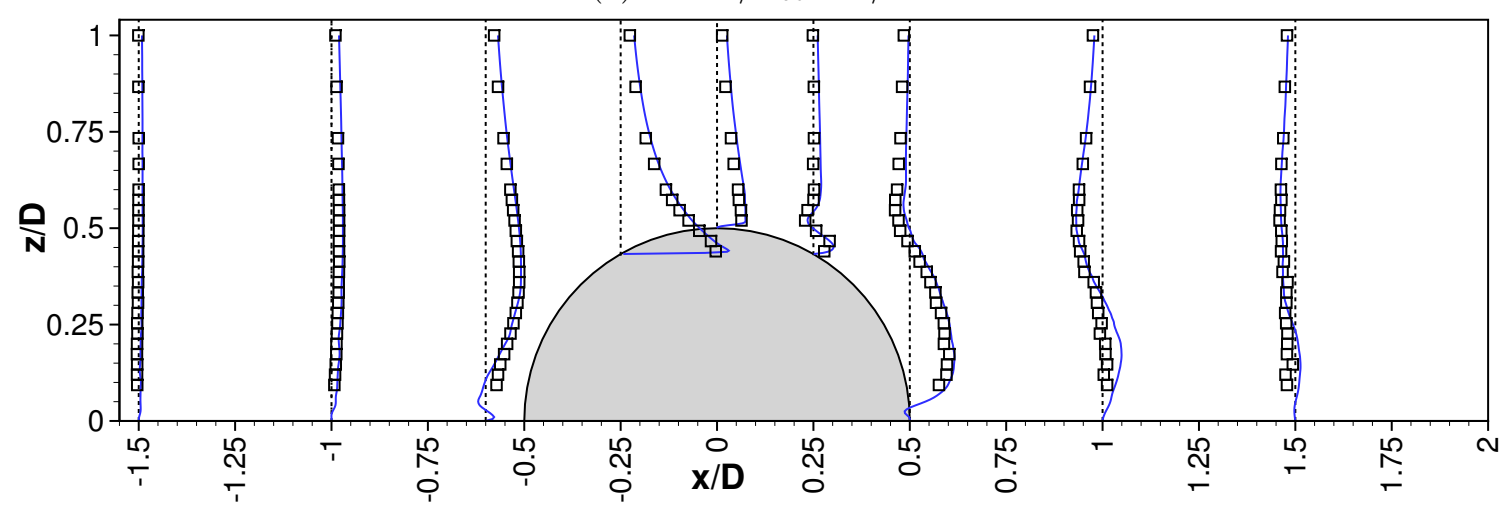

(b) $0.55 \bar{w} / U_{\infty}+x / D$.

Figure 18: Comparison of the experimental (black symbols) and numerical (blue lines) timeaveraged streamwise and wall-normal velocity in the symmetry $x-z$-plane at $y / D=0$ and $x / D=$ $\{-1.5,-1,-0.6,-0.25,0,0.25,0.5,1,1.5\}$ (only every second measurement point is displayed).

the vortical structures as explained in the unsteady flow results (see Section 5). This region stretches out into the upper recirculation region where turbulent mixing is perceived. The "splatting" process arising at the reattachment point produces also streamwise fluctuations. However, these are not visible in the figure, because their associated magnitude is much lower than in the shear layer.

The distribution of the spanwise normal component $\overline{v^{\prime} v^{\prime}} / U_{\infty}^{2}$ is depicted in Figs. 19(c) and (d). The experimental data show high Reynolds stresses throughout the recirculation area and the near-wall region including the reattachment point. Note that the spanwise normal component $\overline{v^{\prime} v^{\prime}} / U_{\infty}^{2}$ around the reattachment area is very high. Its value is comparable with the normal Reynolds stress $\overline{u^{\prime} u^{\prime}} / U_{\infty}^{2}$ in the shear layer. It is assumed that the spanwise velocity fluctuations are associated with the "splatting" process taking place in the reattachment region and with the detaching vortices at the sides of the hemisphere. Near the reattachment point the flow hits the wall and a part of the momentum is redistributed from the wall-normal component to the lateral component. Moreover, the vortices detaching at the sides of the hemisphere (as shown in Section 5) additionally cause a lateral oscillating motion of the recirculation area. This particular movement is related to the "von Kármán"-shedding processes at the lower sides of the hemisphere. The results of the large-eddy simulation support these observations delivering higher normal Reynolds stresses in the lower wake flow. Nevertheless, the experimental results show a significantly higher $\overline{v^{\prime} v^{\prime}} / U_{\infty}^{2}$ distribution in the upper part of the recirculation compared with the numerical simulation. The reason for this deviation is presently unclear.

The wall-normal Reynolds stress $\overline{w^{\prime} w^{\prime}} / U_{\infty}^{2}$ is presented in Figs. 19(e) and (f). Once more the 


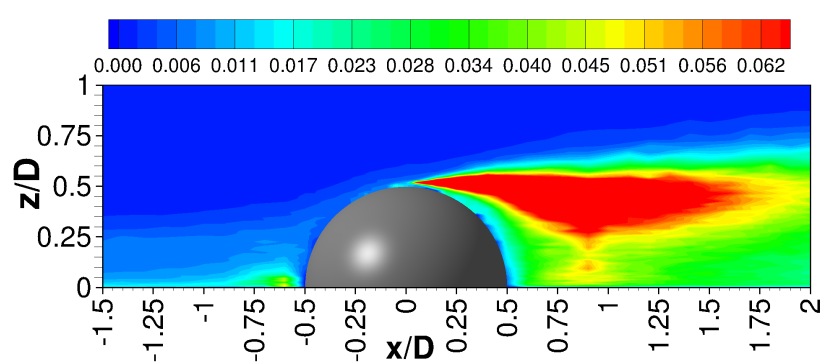

(a) $\overline{u^{\prime} u^{\prime}} / U_{\infty}^{2}(\mathrm{LDA})$.

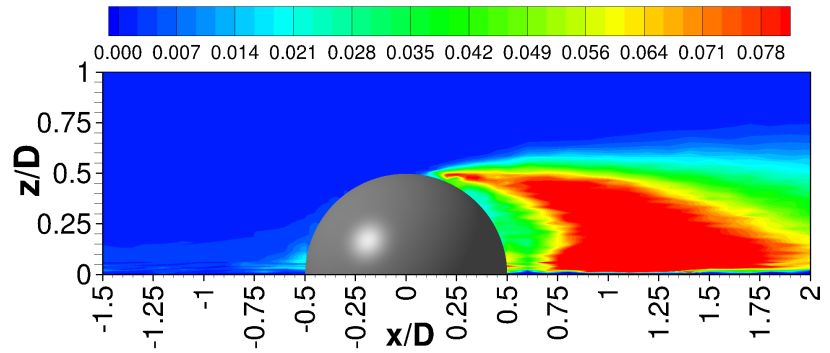

(c) $\overline{v^{\prime} v^{\prime}} / U_{\infty}^{2}(\mathrm{LDA})$.

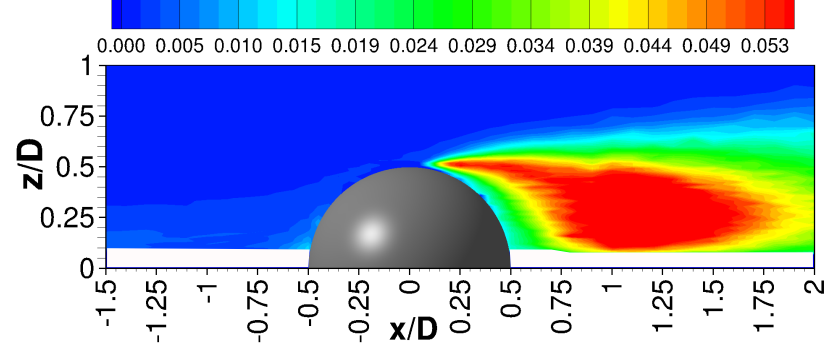

(e) $\overline{w^{\prime} w^{\prime}} / U_{\infty}^{2}(\mathrm{LDA})$.

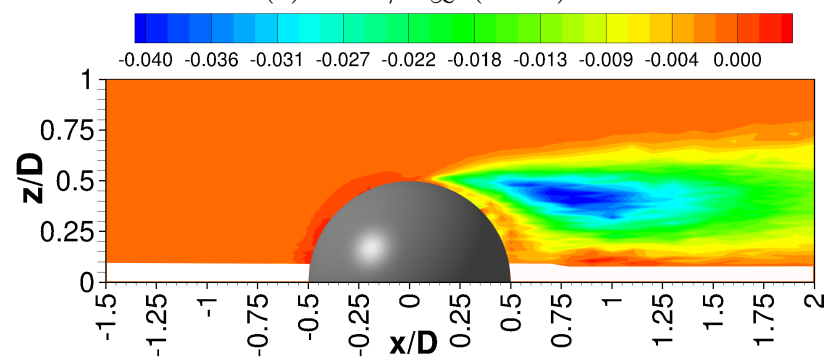

(g) $\overline{u^{\prime} w^{\prime}} / U_{\infty}^{2}(\mathrm{LDA})$.

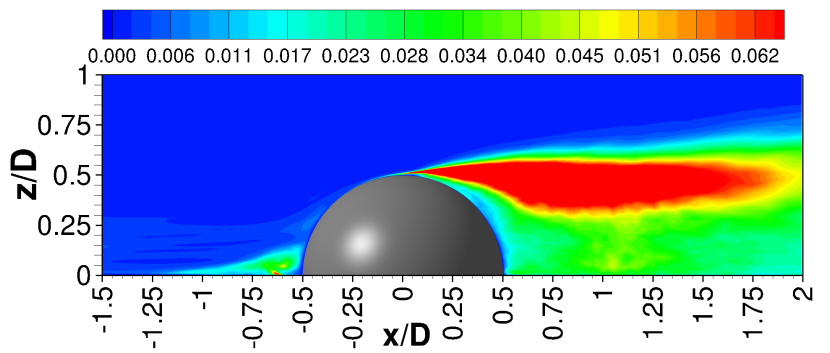

(b) $\overline{u^{\prime} u^{\prime}} / U_{\infty}^{2}$ (LES).

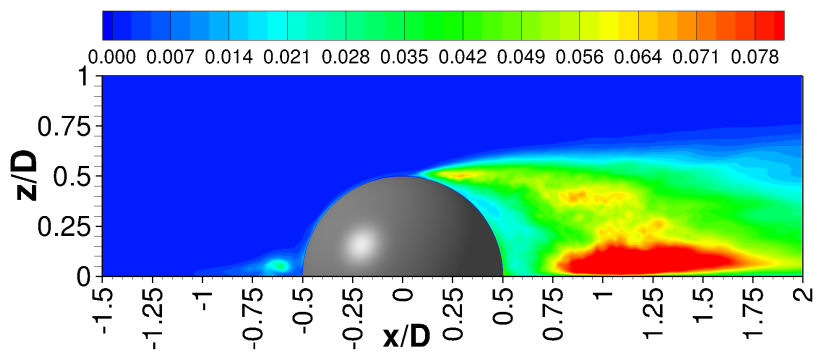

(d) $\overline{v^{\prime} v^{\prime}} / U_{\infty}^{2}$ (LES).

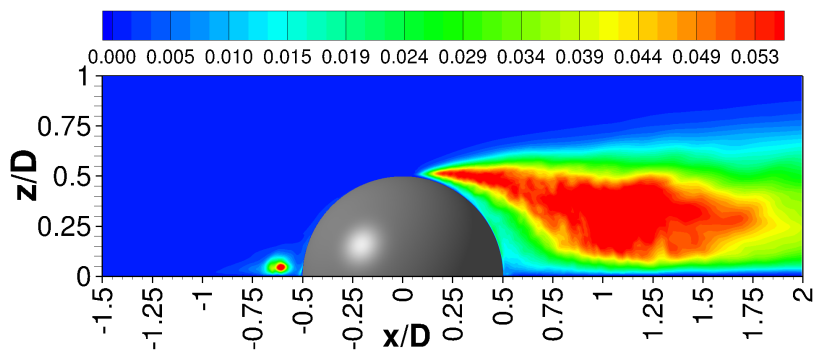

(f) $\overline{w^{\prime} w^{\prime}} / U_{\infty}^{2}$ (LES).

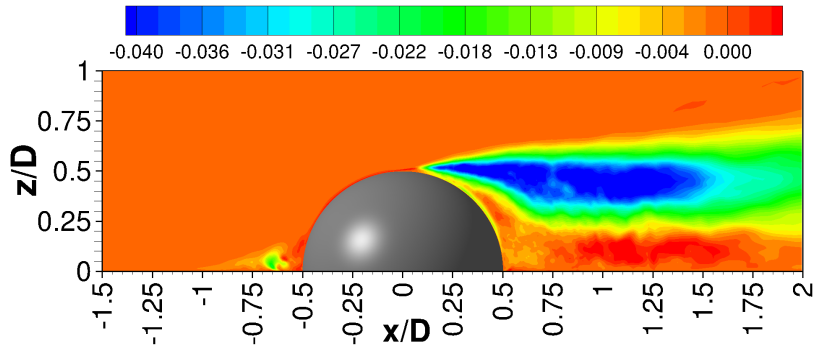

(h) $\overline{u^{\prime} w^{\prime}} / U_{\infty}^{2}$ (LES).

Figure 19: Comparison of the experimental and numerical time-averaged Reynolds stresses in the symmetry $x-z$-plane at $y / D=0$.

LES predictions are in good agreement with the measurement except for the oncoming area, where the turbulence intensity is slightly damped. Intense Reynolds stresses are present in the free shear layer and the recirculation region at $1 \leq x / D \leq 1.5$.

The Reynolds shear stress $\overline{u^{\prime} w^{\prime}} / U_{\infty}^{2}$ is shown in Figs. 19(g) and (h). Both the measurement and the simulation show that the largest values are expected in the free shear layer.

The profiles of the Reynolds stresses are presented in Fig. 20. The complete upper flow field until $x / D=0$ shows only minor differences between the laser-Doppler measurements and the large-eddy simulation for all Reynolds stress components. The streamwise Reynolds stress $\overline{u^{\prime} u^{\prime}} / U_{\infty}^{2}$ is well predicted past the separation point. As already mentioned there are some discrepancies in the spanwise normal Reynolds stresses $\overline{v^{\prime} v^{\prime}} / U_{\infty}^{2}$ in the wake regime. It is not quite clear yet whether the numerical simulation underestimates the level in the upper regime of the recirculation area or if the measurement of configuration 1 overpredict these values. The 
Flow, Turbulence and Combustion, vol. 97 (1), pp. 79-119, (2016).

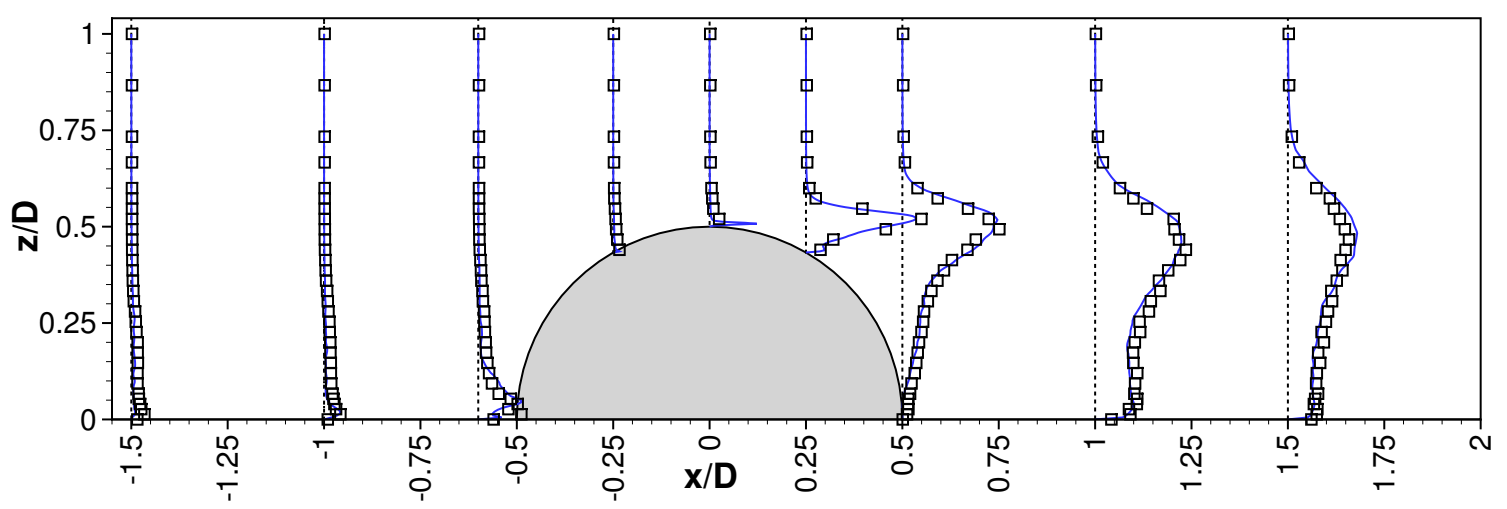

(a) $2.5 \overline{u^{\prime} u^{\prime}} / U_{\infty}^{2}+x / D$.

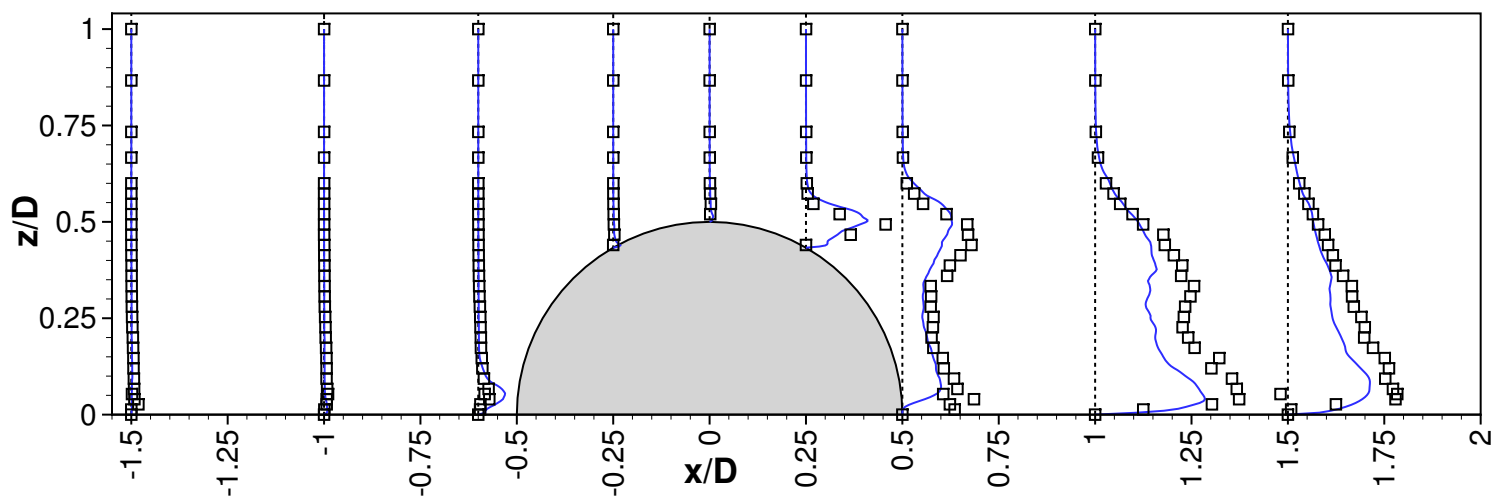

(b) $2.5 \overline{v^{\prime} v^{\prime}} / U_{\infty}^{2}+x / D$.

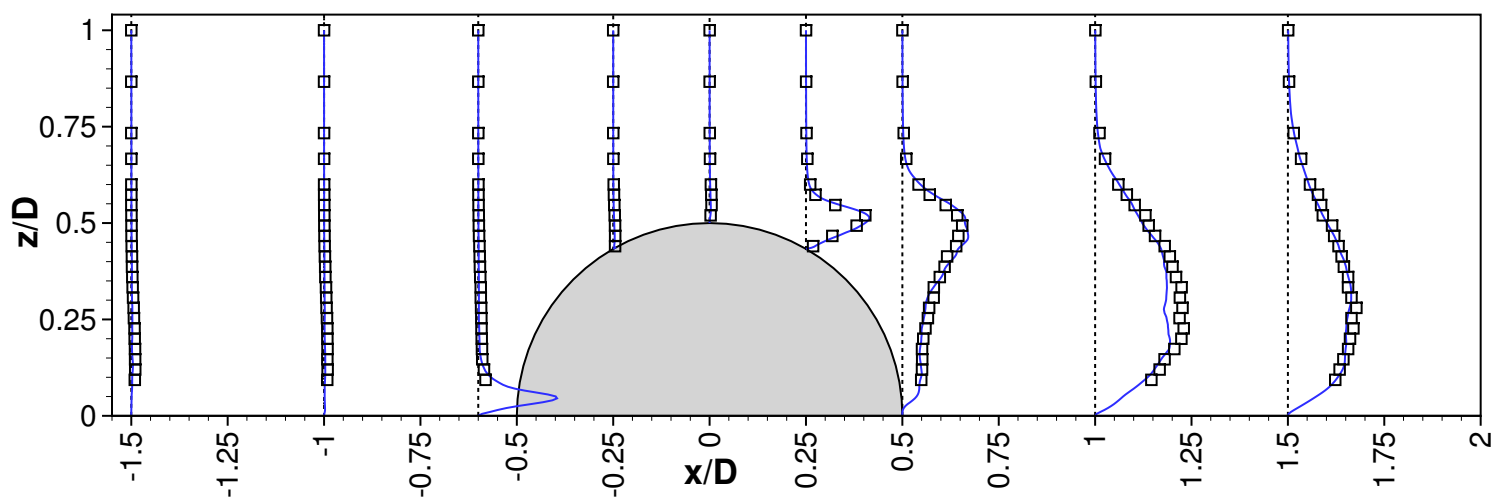

(c) $3 \overline{w^{\prime} w^{\prime}} / U_{\infty}^{2}+x / D$.

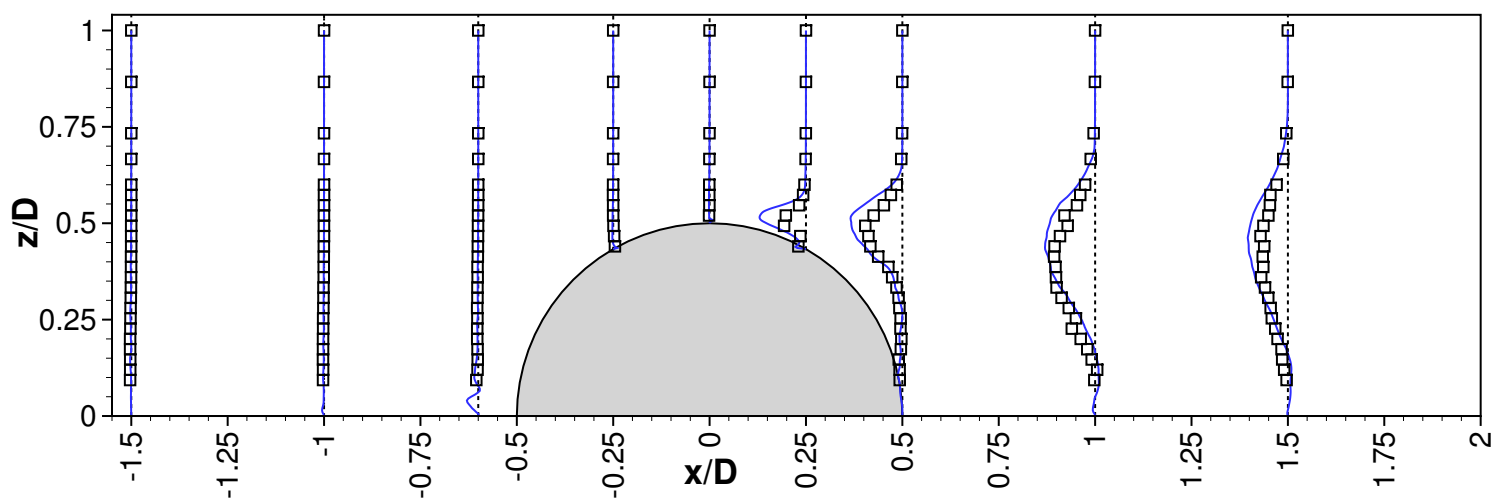

(d) $3 \overline{u^{\prime} w^{\prime}} / U_{\infty}^{2}+x / D$.

Figure 20: Comparison of the experimental (black symbols) and numerical (blue lines) time-averaged Reynolds stresses in the symmetry $x-z$-plane at $y / D=0$ and $x / D=\{-1.5,-1,-0.6,-0.25,0,0.25,0.5,1,1.5\}$ (only every second measurement point is displayed). 
Flow, Turbulence and Combustion, vol. 97 (1), pp. 79-119, (2016).

results of the Reynolds shear stress $\overline{u^{\prime} w^{\prime}} / U_{\infty}^{2}$ are in close agreement between the experiment and the simulation.

\subsection{Spanwise plane}

Besides the analysis of the symmetry plane, measurements are also conducted for the spanwise flow at a $y-z$ cross-section as briefly mentioned in Section 3.2.1. It is processed using LDA configuration 1 (see Fig. 5(a)) which offers the possibility of an appropriate near-wall resolution across the complete spanwise extension of the test section. The motivation for this measurement is to complement the data obtained from the symmetry plane in order to receive a better three-dimensional perspective of the flow field and to compare it with the large-eddy simulation. The investigation focuses on the streamwise and spanwise flow components at the position $x / D=0.5$ ( $y-z$-plane) behind the hemisphere.

\subsubsection{Characteristics of the flow field}

The color plots for the streamwise component are presented in Figs. 21(a) and (b). The chosen position of the spanwise plots provides an insight into the specific velocity distribution in the wake regime: The uniform outer flow field, the accelerated flow above the hemisphere, the shear layer distribution, the recirculation zone and the near-wall flow at the far sides. First, an almost symmetric velocity distribution of the streamwise flow component with regard to the symmetry plane at $y / D=0$ is recognizable. The shear layer forms an arch-type structure that is related to the roll-up process of the detaching vortices. The recirculation region expands from $-0.3 \leq y / D \leq 0.3$ and $0 \leq z / D \leq 0.3$. In the near-wall region the centers of the trailing necklace vortices are observed at the position $y / D= \pm 0.7$. Overall the distribution and the magnitude of the streamwise velocity component are confirmed by the numerical results.

Figures 21(c) and (d) refer to the spanwise velocity component. The velocity distribution in the lower region closely behind the hemisphere is dominated by two counter-rotating vortices that are located symmetrically to the plane $y / D=0$. The alternating direction of the velocity component across the spanwise direction indicates the counterwise rotation of the vortices.

\subsubsection{Reynolds stresses}

Referring to the discussed velocity distributions for the spanwise plane, the corresponding Reynolds stresses are depicted in Fig. 22. Figures 22(a) and (b) show the streamwise Reynolds stress component $\overline{u^{\prime} u^{\prime}} / U_{\infty}^{2}$. The minor differences in its size between the experiment and the large-eddy simulation is related to the applied grid resolution. The very fine mesh used in the large-eddy simulation leads to a better resolution of the gradients in the flow field. This can easily be perceived by the Reynolds stress distribution in the shear layer that reveals an overall thinner arch. The near-wall data of the experiment between $0 \leq z / D \leq 0.02$ are erroneous due to optical reflections of the flat plate that occur in the utilized LDA setup (configuration 1) and are therefore not usable for further flow interpretation.

Finally, a view of the spanwise Reynolds stress distribution is given in Figs. 22(c) and (d) which confirms all significant effects already mentioned for the streamwise case. Additionally, this component has noticeably higher Reynolds stresses located in the region $-0.15 \leq y / D \leq 0.15$ compared to the streamwise Reynolds stresses. This seems to be connected to the two large vortices that form in this region mentioned in Section 6.2.1 which are connected to a strong spanwise movement. As observed in the case of the symmetry plane the Reynolds stresses in the experiment are more pronounced. This effect can be explained by the relatively coarse two-dimensional orthogonal measurement grid of the laser-Doppler data that leads to a slightly distorted image of the spanwise Reynolds stresses which are perceived as a horizontal "stripepattern" of the fluctuations. On the contrary, the large-eddy simulation reveals its capability of 


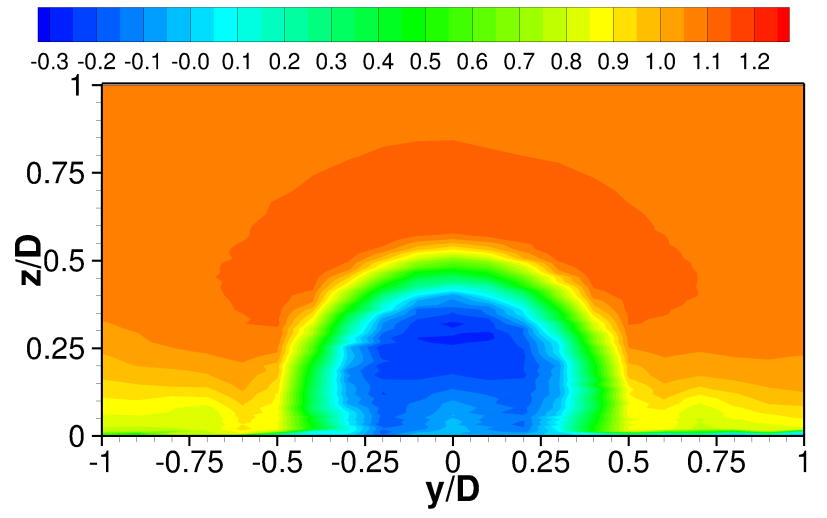

(a) $\bar{u} / U_{\infty}(\mathrm{LDA})$.

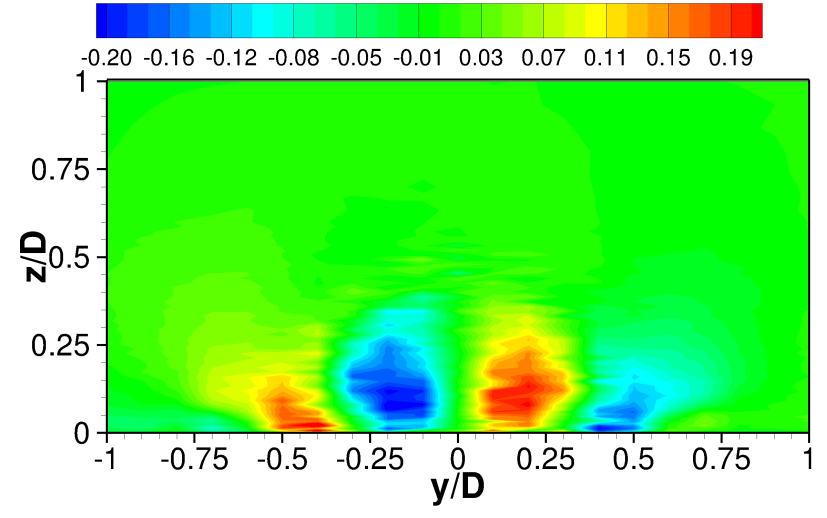

(c) $\bar{v} / U_{\infty}(\mathrm{LDA})$.

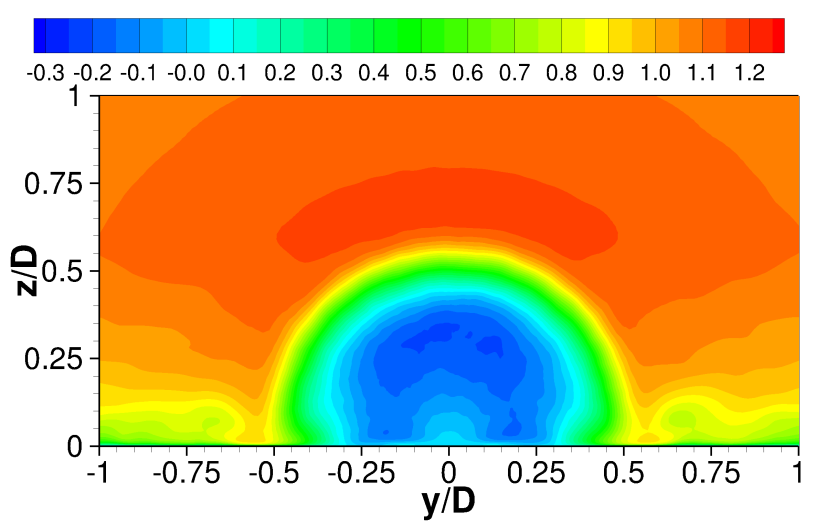

(b) $\bar{u} / U_{\infty}$ (LES).

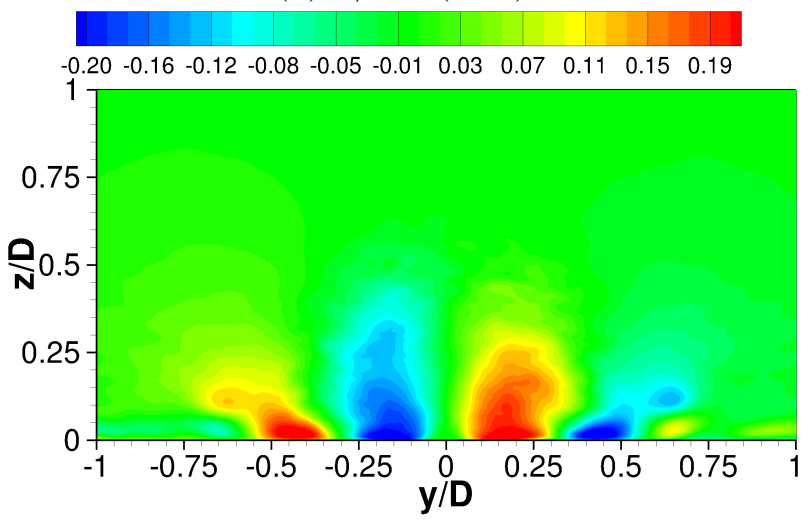

(d) $\bar{v} / U_{\infty}$ (LES).

Figure 21: Comparison of the experimental and numerical time-averaged velocity components in the $y-z-$ plane at $x / D=0.5$.

a more detailed spatial view as a result of the fine three-dimensional numerical mesh. Nevertheless, the discrepancies in the spanwise Reynolds stresses between the numerical simulation and the experiment should be examined in further studies to clarify which side of the investigation is causing this deviation.

\section{3. $3 D$ visualization of the time-averaged flow}

The major benefit of the large-eddy simulation lies in its high spatio-temporal resolution. This leads to a large amount of flow field information that can be utilized to analyze even smallest flow structures in characteristic regions such as corner eddies. Besides this, large structures, like the horseshoe vortex system, can be explored in detail. A few chosen numerical results of the three-dimensional time-averaged flow field shall provide a deeper insight into the characteristics of the flow field around the hemisphere.

Figure 23 presents the bottom wall streamlines based on the time-averaged velocity in the $x-$ $y$-plane including the surface of the hemisphere. This view is used to examine the separation and reattachment behavior of the flow field:

- Far upstream of the hemisphere the flow is divided by the separation streamline that wriggles widely around the obstacle and is connected to the separation of the boundary layer from the ground. This phenomenon is also observed by Martinuzzi and Tropea [9] for the turbulent flow past a wall-mounted cube at $\mathrm{Re}=4.0 \times 10^{4}$.

- The upstream region close to the hemisphere is dominated by the horseshoe vortex system. At certain positions along the symmetry plane an alternating series of saddle and 


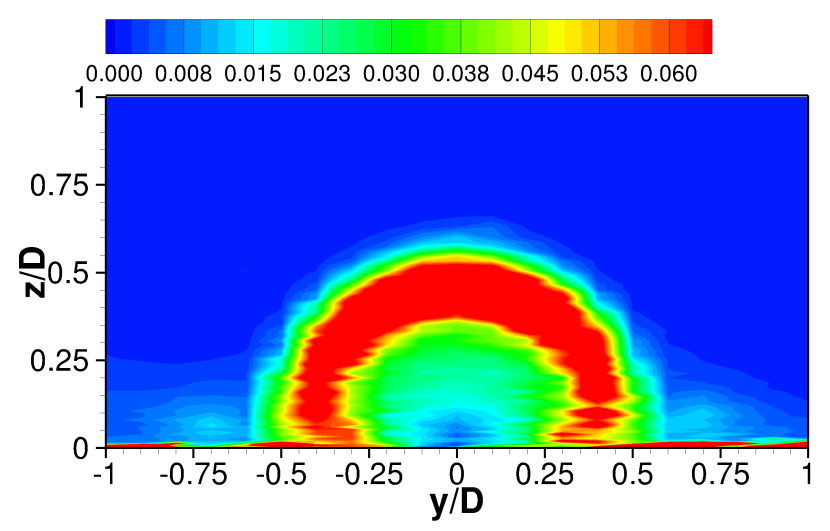

(a) $\overline{u^{\prime} u^{\prime}} / U_{\infty}^{2}(\mathrm{LDA})$.

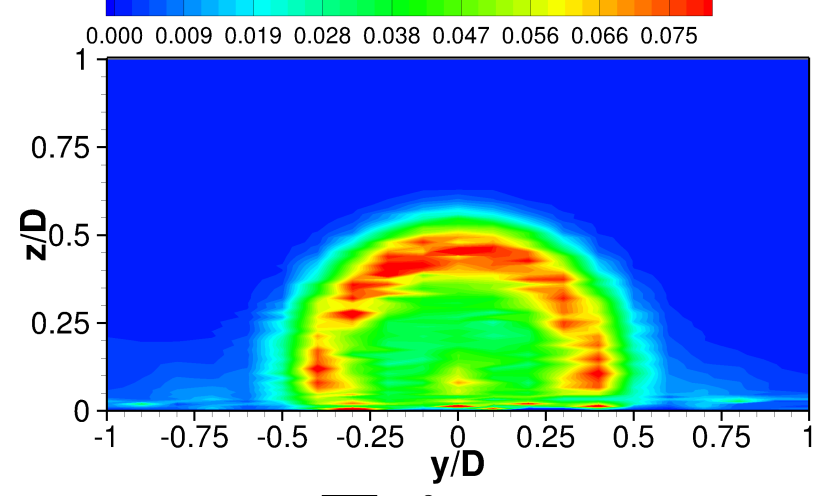

(c) $\overline{v^{\prime} v^{\prime}} / U_{\infty}^{2}(\mathrm{LDA})$.

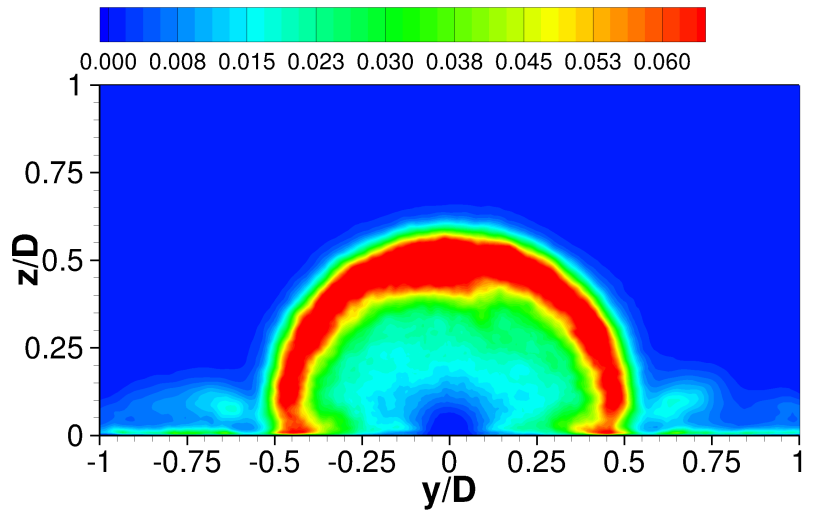

(b) $\overline{u^{\prime} u^{\prime}} / U_{\infty}^{2}$ (LES).

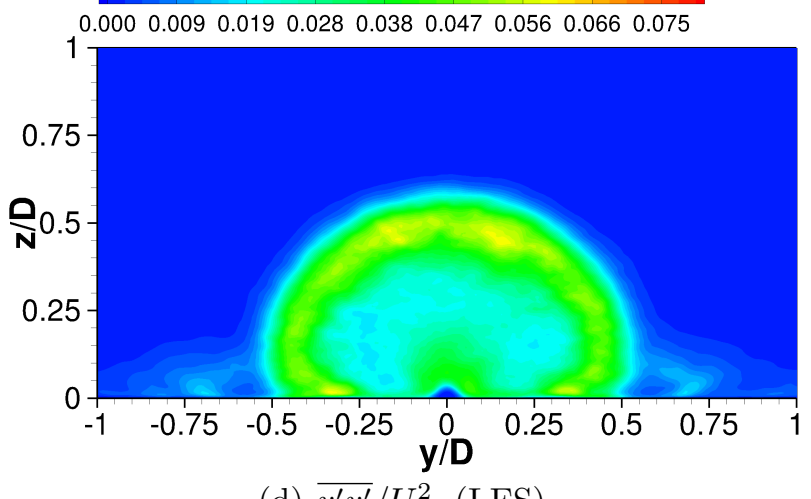

(d) $\overline{v^{\prime} v^{\prime}} / U_{\infty}^{2}$ (LES).

Figure 22: Comparison of the experimental and numerical time-averaged Reynolds stresses in the $y-z-$ plane at $x / D=0.5$.

nodal points indicates either a separation or an reattachment of the flow and helps to separate single vortices. The points can be easily detected since the streamlines bundle up at these specific spots. A comparable formation of vortices is noticed for the wall-mounted cube [9].

- In front of the hemisphere after the stagnation point, the flow field accelerates along the surface up to the separation line. This separation line stretches out along the circumference nearly up to the bottom wall. This is a significant difference to the turbulent flow past the axisymmetric bump $[14-16,24]$ at $R e=1.3 \times 10^{5}$ (based on the hill height), where the separation line is shifted to the backside of the $3 \mathrm{D}$ hill.

- Behind the obstacle, a classical recirculation area forms with a reattachment point located in the symmetry plane. In the recirculation area two symmetric spiral flow patterns are visible on the ground, which represent the footprint of the arch-type vortical structure. These pattern are also observable for other wall-mounted bluff obstacles such as the cube [9] and the finite-height circular cylinder [10]. Such an arch-type structure is not mentioned in the discussion of the axisymmetric 3D hill flow, but spiral flow patterns are also present in the separation region, which in this case is however much thinner and shorter [14-16, 24].

One of the characteristic regions is the horseshoe vortex system. Figure 24 depicts the streamlines in the symmetry plane just in front of the lower base of the hemisphere to highlight the vortices forming the horseshoe vortex system. These vortices are paired. The vortices of 
a pair rotate in opposite directions. Each vortex of the system is classified by applying the indices chosen by Baker [56] who, among others, presented the mechanism of the formation of the complex vortex system. Vortex 0 is related to the separation of the boundary layer just in front of the lower face of the hemisphere. The counter-rotating vortex $0^{\prime}$ is also resolved in the LES but too small to be visualized in Fig. 24. It is followed by the primary vortex 1 which is the largest structure of the horseshoe vortex system. It is caused by the separation of the boundary layer due to the presence of vortex 0 . A secondary counter-rotating vortex $1^{\prime}$ is generated by the detaching boundary layer beneath the primary vortex. This complex separation process leads to an overall number of four visible vortices. An additional view of the upstream vortex system is given in Fig. 25 as three-dimensional filaments. It relates to the $x-y$-plane view of the hemisphere and shows the first four previously described vortices as they trail around the front of the hemisphere. Due to its symmetry only one side of the horseshoe vortex distribution is presented. The illustration in Fig. 26 enhances the primary vortex with the help of 3D-filaments based on the time-averaged velocity and colored by the mean wall-normal velocity.

The second major flow region is the recirculation area visualized in Fig. 27. The illustrated 3Dfilaments in the near wake are coiled up in an arc-shaped structure that characterizes the size of the recirculation area. Corresponding wall streamlines of the rear side of the hemisphere are given in Fig. 28. The streamlines depict the large backflow area on the surface of the hemispherical body. Two other symmetric spiral flow patterns appear on each side relating to the lateral separation points. A narrow and flat counter-rotating vortical structure that stretches along the circumferential direction is presented in Fig. 29. The close up view of the symmetry plane at the top of the hemisphere reveals this flat region that can also be observed in Fig. 23.

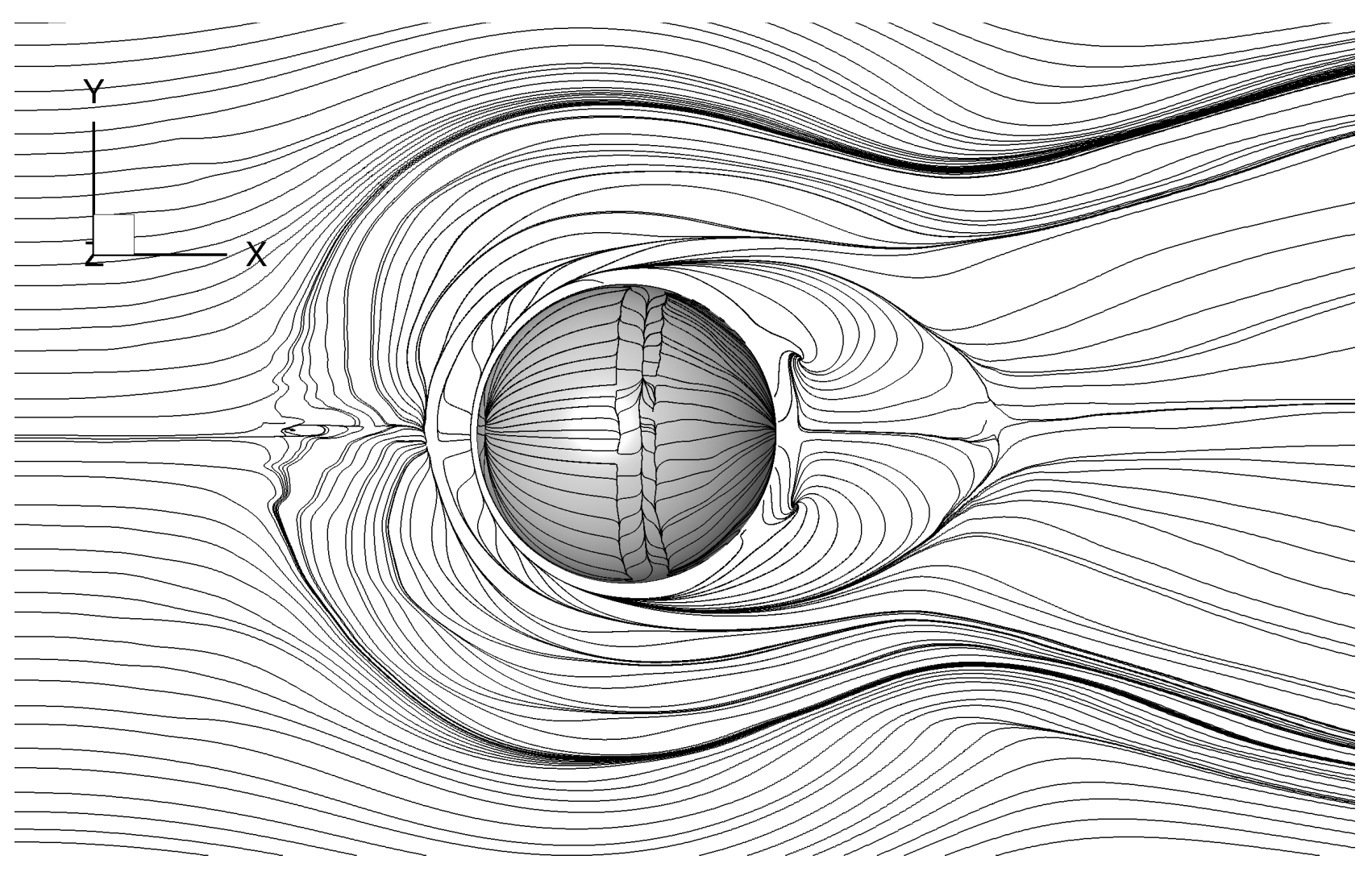

Figure 23: Time-averaged streamlines near the bottom wall and on the surface of the hemisphere. 
Flow, Turbulence and Combustion, vol. 97 (1), pp. 79-119, (2016).

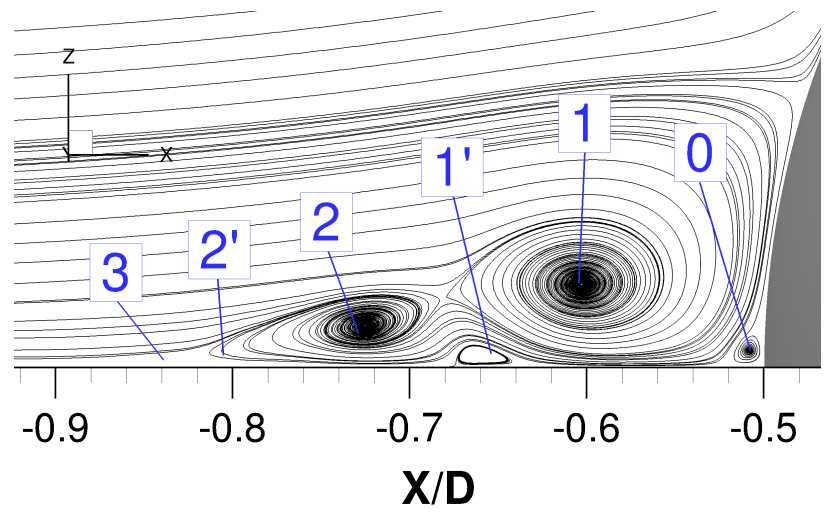

Figure 24: Horseshoe vortex system: Time-averaged streamlines in the symmetry plane upstream of the hemisphere.

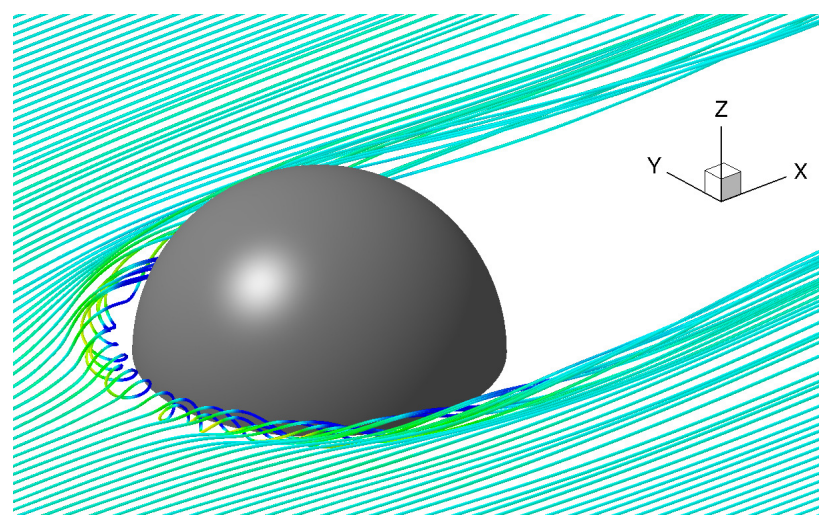

Figure 26: Horseshoe vortex system: Vortex 1 visualized by $3 \mathrm{D}$-filaments based on the time-averaged velocity and colored by the mean wall-normal velocity.

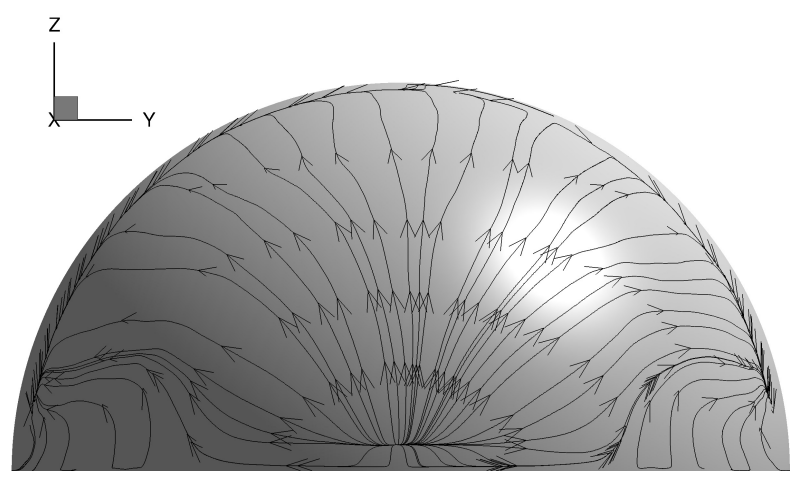

Figure 28: Time-averaged streamlines on the surface behind the hemisphere.

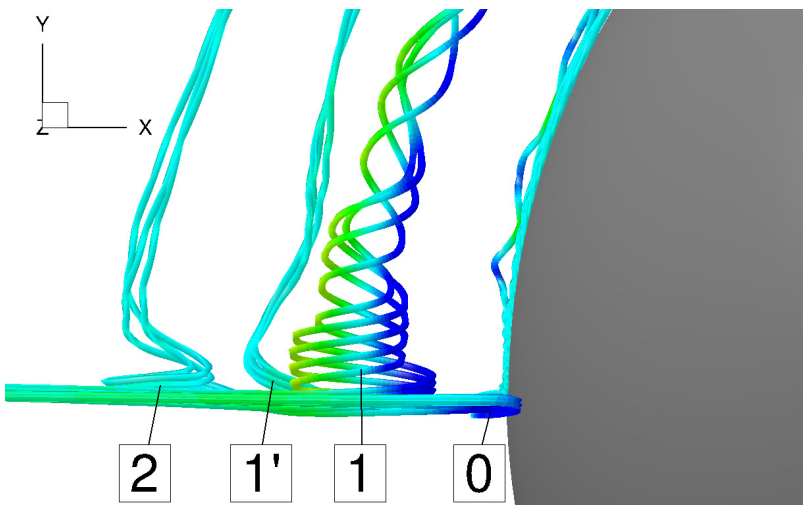

Figure 25: Horseshoe vortex system: 3D-filaments based on the time-averaged velocity and colored by the mean wall-normal velocity.

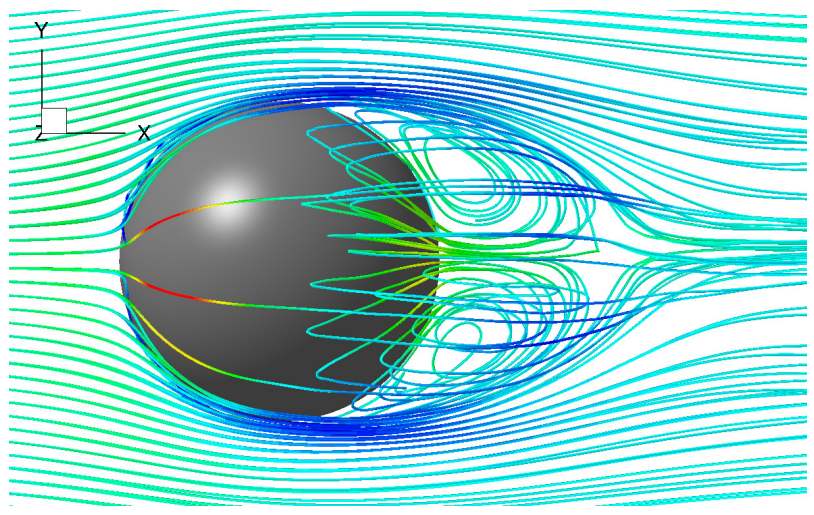

Figure 27: Recirculation area: 3D-filaments based on the time-averaged velocity and colored by the mean wall-normal velocity.

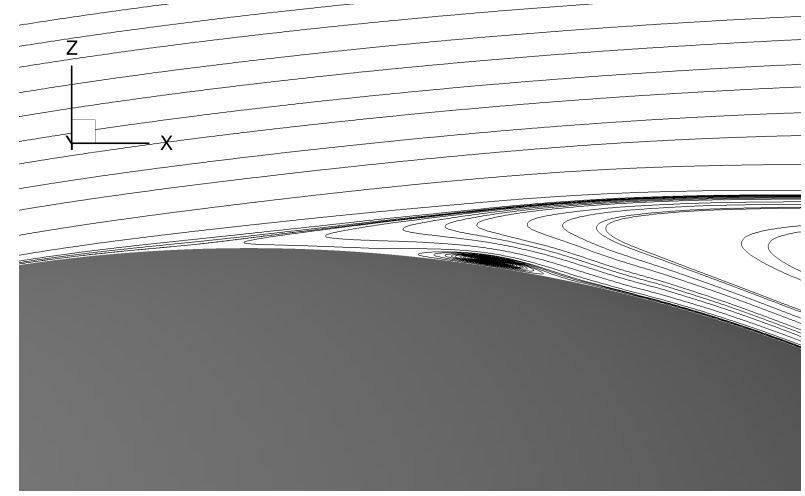

Figure 29: Separation line: Time-averaged streamlines in the symmetry plane close to the apex of the hemisphere. 
Flow, Turbulence and Combustion, vol. 97 (1), pp. 79-119, (2016).

\section{Conclusions}

The present study outlines a comparison between experimental and numerical investigations of a hemispherical body in a thick turbulent boundary layer at $R e=50,000$. Comprehensive laser-Doppler measurements are conducted in a wind tunnel with an open test section to acquire the three-dimensional flow field around the hemisphere. To achieve the desired boundary layer characteristics a specialized arrangement of turbulence generators is mounted upstream of the test section. Additionally, a constant temperature anemometer is applied to obtain the power spectral density at specific locations in the wake flow. The numerical predictions are conducted using the large-eddy simulation technique. In order to gain optimum comparability between the experiment and the numerical simulation, the statistical data of the wind tunnel measurements for the boundary layer are used to generate appropriate inflow conditions for the LES. For this purpose, the approaching boundary layer is generated by superimposing the turbulent mean velocity profile with spatio-temporal fluctuations provided as source terms by a synthetic turbulence inflow generator reproducing the turbulent characteristics of the flow. With almost identical inflow conditions mimicking the approaching boundary layer quite well, the comparison of the time-averaged flow field in the symmetry and a cross-flow plane reveals an excellent agreement between LDA measurements and LES predictions except for the spanwise Reynolds stresses which need clarification.

The experimental and numerical time-averaged results, including mean velocity distributions and Reynolds stresses, are used for the validation of the numerical simulation. Due to its superior spatio-temporal resolution compared to the measurements the validated large-eddy simulation offers the advantage to explore the unsteady features of the complicated flow. An exception is the measurement of the power spectrum. Due to the very small computational time step used in the LES, the constant temperature anemometry still offers a more practical way to gather an adequate amount of data in a reasonable time. Combining both, a detailed analysis of the complex three-dimensional flow phenomena can be processed.

The first part of the results presents the unsteady flow characteristics with major focus on the shedding processes in the wake regime and their interactions. Along the circumference in the $y$-z-plane the shedding type differs: On the upper area the rolled-up structures separate at a frequency in the range of $7.9 \mathrm{~Hz} \leq f_{1} \leq 10.6 \mathrm{~Hz}\left(0.23 \leq \mathrm{St}_{1}=f_{1} D / U_{\infty} \leq 0.31\right)$. Frequently, a merging of distinct smaller structures is observed just after the separation that leads to larger vortices. At the lower sides of the hemisphere alternating or symmetric shedding of bigger vortices is detected at a frequency of $f_{2}=5.5 \mathrm{~Hz}\left(\mathrm{St}_{2} \approx 0.16\right)$. All together it forms a chain of entangled vortical "hairpin"-structures of different sizes traveling downstream.

The second part of the results contains the time-averaged data. It is used to identify characteristic regions within the flow field such as the horseshoe vortex, separation/stagnation points and the wake. Far upstream, the flow is divided by an outer streamline, which widely surrounds the structure. Upstream and close to the hemisphere the horseshoe vortex dominates. For the present case it contains four paired vortices, which counter-rotate to each other. After the stagnation point the flow accelerates up to the separation line. A classical large recirculation area forms behind the body, containing an arch-type vortical structure. For the current turbulent regime a narrow and flat counter-rotating vortical region is also present along the circumferential direction just after this separation line.

The turbulence intensity of the approaching inflow has a large impact on the extensions and location of the mentioned phenomena. This is for example obvious based on the size and intensity of the horseshoe vortex system upstream of the hemispherical structure which significantly depends on the level of turbulent fluctuations of the approaching boundary layer. Without the 
inflow turbulence this region is largely overestimated by the large-eddy simulation. The influence of the turbulence level at the inlet can also be observed in the location of the separation line which moves to a position further downstream when the turbulence intensity increases resulting in a shorter recirculation area. An additional study investigates the influence of different subgrid-scale models on the results. As an outcome only minor deviations between the results relying on different SGS models are found due to the very fine grid used in the LES. The results of the present study form the basis for the upcoming investigations that move towards fluid-structure interaction. Future experimental and numerical studies intend to exchange the rigid structure by a thin-walled flexible membranous hemisphere. Air inflated structures as depicted in Fig. 1(a) indicate the practical value of such examinations.

\section{Acknowledgments}

The numerical part of the project is financially supported by the Deutsche Forschungsgemeinschaft under the contract number BR 1847/12-1. The computations were carried out on the German Federal Top-Level Computer SuperMUC at LRZ Munich under the contract number pr84na. Furthermore, the authors want to thank Markus Klein (Universität der Bundeswehr München) for providing the original source code of the digital filter based inflow procedure. 
Flow, Turbulence and Combustion, vol. 97 (1), pp. 79-119, (2016).

APPENDIX A POSITION OF THE VORTEX GENERATORS

\section{Appendix A. Position of the vortex generators}

The positions of the utilized vortex generators based on the diameter of the hemisphere are given in Fig. A.30. The triangular spikes are raised at an angle of $50^{\circ}$ related to the bottom of the nozzle.

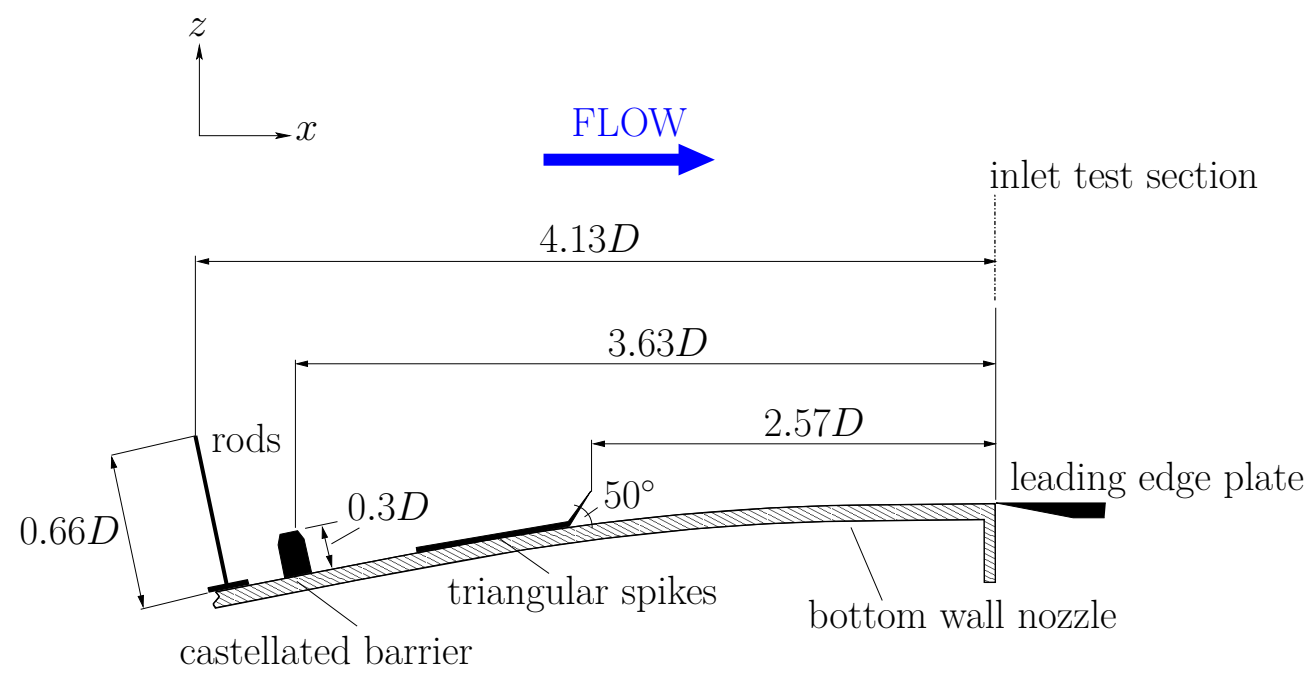

Figure A.30: Position of the vortex generators inside the nozzle. 
Flow, Turbulence and Combustion, vol. 97 (1), pp. 79-119, (2016).

APPENDIX B INFLUENCE OF THE SUBGRID-SCALE MODEL

\section{Appendix B. Influence of the subgrid-scale model}

In order to evaluate the influence of the SGS model on the current case, five simulations are carried out with different SGS models:

- Smagorinsky (Smag.) model [46] with three different constants $C_{s}=0.065,0.1$ and 0.18

- Dynamic Smagorinsky (Dyn.) model [29];

- WALE model [28] with a constant $C_{W}=0.33$.

These preliminary computations use a slightly different numerical setup: A constant inlet velocity profile ( $1 / 7$ power law) is considered without any turbulent fluctuations and no perturbation is added as source term at $x / D=-1.5$. The rest of the setup is identical to the one described in Section 4. The results are time-averaged during about $t^{*}=t U_{\infty} / D=45$ in dimensionless form.

Figure B.31 compares the time-averaged streamwise velocity component $\bar{u} / U_{\infty}$ in the symmetry plane $y=0$ and the time-averaged streamlines near the bottom wall and on the surface of the hemisphere for the tested SGS models. For the sake of brevity more detailed comparisons based on profiles of the time-averaged velocity and Reynolds stresses are not depicted here. To investigate the differences the seven characteristic regions described by Savory and Toy [7] (see Fig. 12) are used:

- The first area is the region of the horseshoe vortices located upstream of the hemisphere. The classical Smagorinsky model with $C_{s}=0.065$ or $C_{s}=0.1$ leads to nearly identical results as the dynamic model. Several counter-rotating vortices are clearly visible in front of the hemisphere. The WALE model delivers the largest vortex similar to Smagorinsky $C_{s}=0.065, C_{s}=0.1$ and to the dynamic model. However, the other smaller vortices of the horseshoe vortex system do not distinctly appear (see Fig. B.32 for a direct comparison). The biggest difference is observed for the classical Smagorinsky model with $C_{s}=0.18$ : The horseshoe vortex system is composed of only one big flat vortex. This difference is clearly visible for the wall streamlines.

- The second region includes the stagnation point. The SGS model has no significant influence on the position of the stagnation point $\left(\theta_{\text {stag }} \approx 158^{\circ}\right)$

- As expected, in the third characteristic region (acceleration area above the hemisphere) the differences between the SGS models are negligible.

- The fourth region comprises the flow separation line on the hemisphere. Its form is identical for all tested SGS models. Based on the angle $\theta_{\text {sep }}$ (see Fig. B.31 for the definition), the position of the flow separation line is the same for all SGS models $\left(\theta_{\text {sep }} \approx\right.$ $96^{\circ}$ ), except for the classical Smagorinsky model with $C_{s}=0.18$. Indeed, this SGS model predicts the flow separation slightly earlier $\left(\theta_{\text {sep }} \approx 95^{\circ}\right)$.

- In the fifth (dividing streamline) and sixth (shear layer) region the influence of the SGS model is hardly visible. Form and position of both regions are nearly identical.

- The last characteristic region is the reattachment point with respect to the symmetry plane. All simulations predict its position at about $x_{\text {reattach }} / D=1.35$. 
To conclude the classical Smagorinsky model with $C_{s}=0.065$ or $C_{s}=0.1$ leads to nearly identical results as the dynamic model. The WALE model predicts similar results as the dynamic one, except in the horseshoe vortex region. Applying the classical Smagorinsky model with $C_{s}=0.18$, several of the characteristic regions show significant differences compared to the dynamic model. Therefore, the classical Smagorinsky model with $0.065 \leq C_{s} \leq 0.1$ or the dynamic Smagorinsky model can be equivalently used for the current case. As mentioned in Section 4 the classical Smagorinsky model with $C_{s}=0.1$ is set. 
Flow, Turbulence and Combustion, vol. 97 (1), pp. 79-119, (2016).

APPENDIX B INFLUENCE OF THE SUBGRID-SCALE MODEL

(a) Smag.

$C_{s}=0.065$
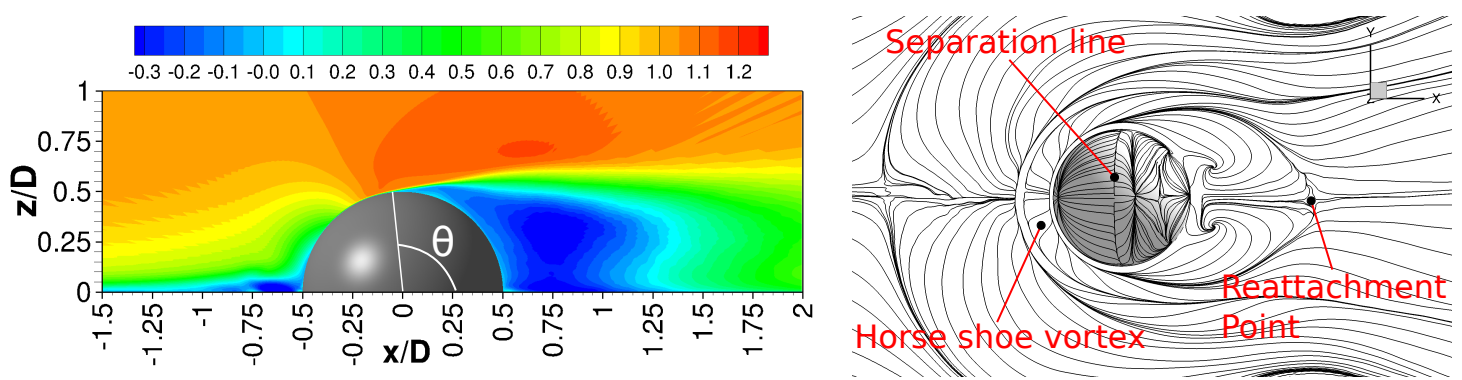

(b) Smag.

$C_{s}=0.1$
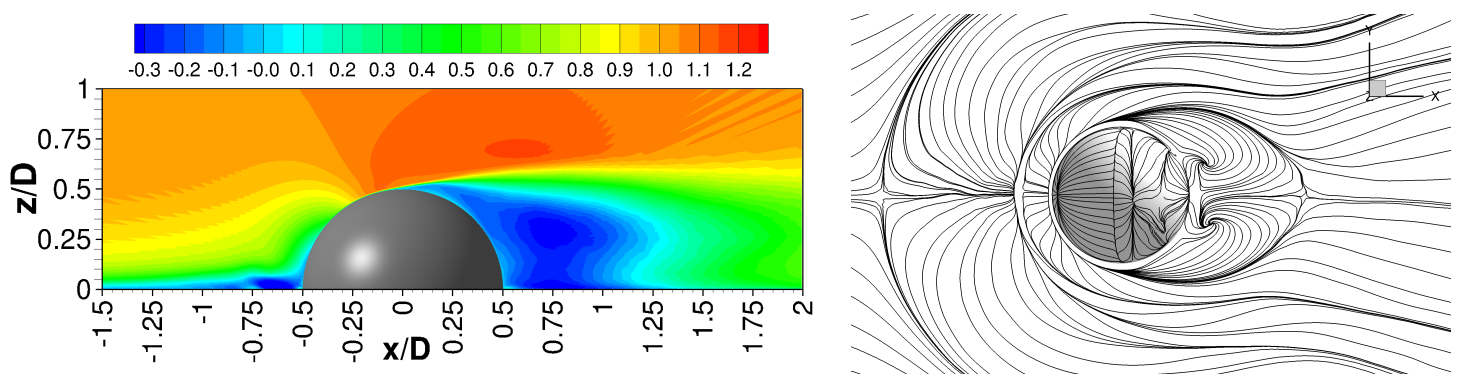

(c) Smag.

$C_{s}=0.18$
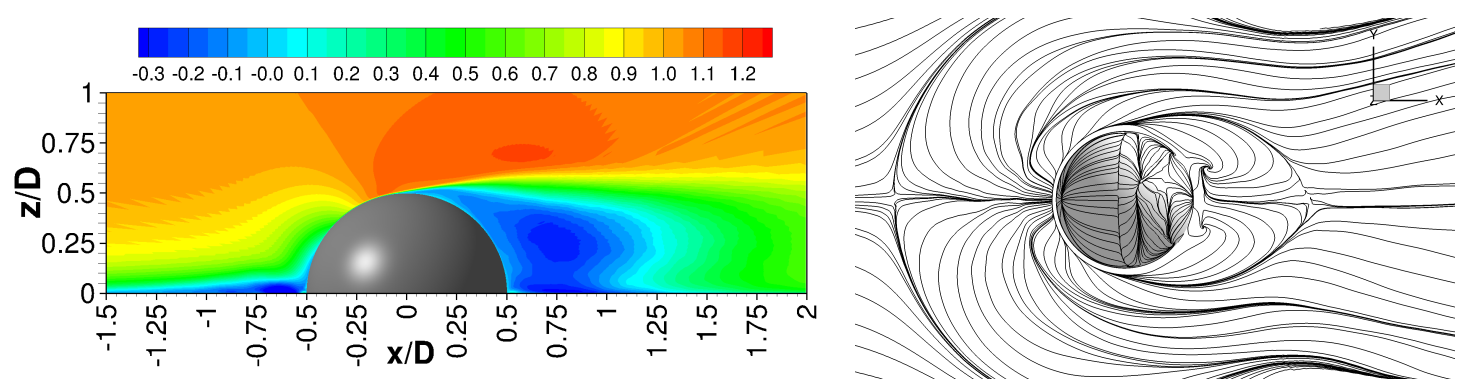

(d) Dyn.
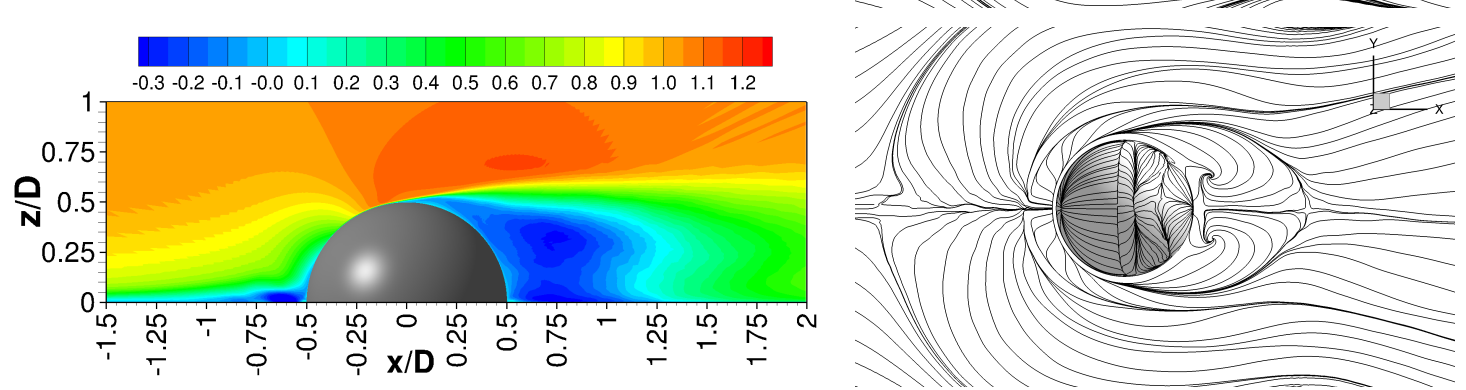

(e) WALE

$C_{W}=0.33$
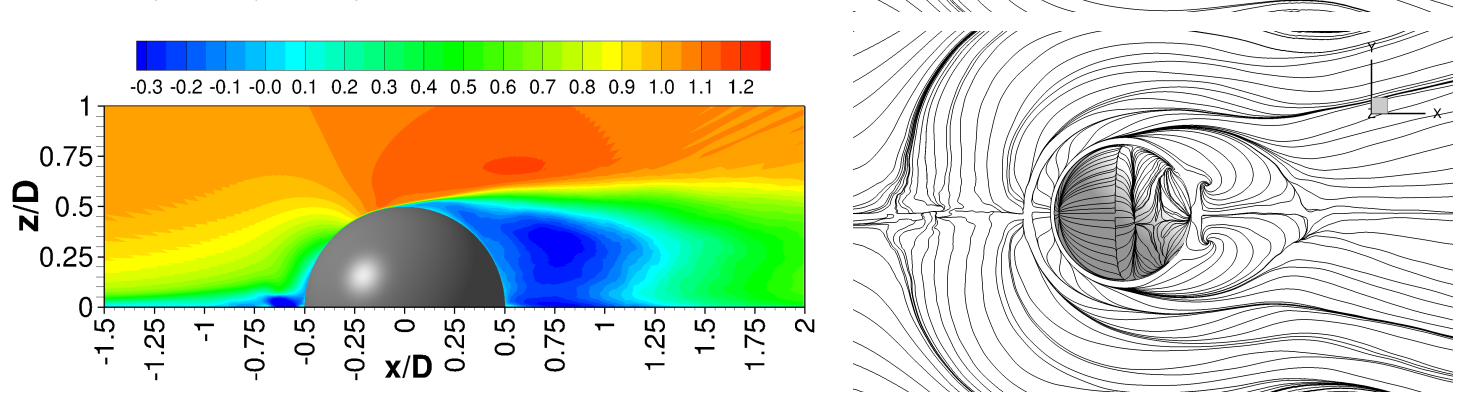

Figure B.31: Influence of different SGS models on the flow: Time-averaged streamwise velocity component $\bar{u} / U_{\infty}$ at the symmetry plane $y=0$ (left), time-averaged streamlines near the bottom wall and on the surface of the hemisphere (right). 
Flow, Turbulence and Combustion, vol. 97 (1), pp. 79-119, (2016).

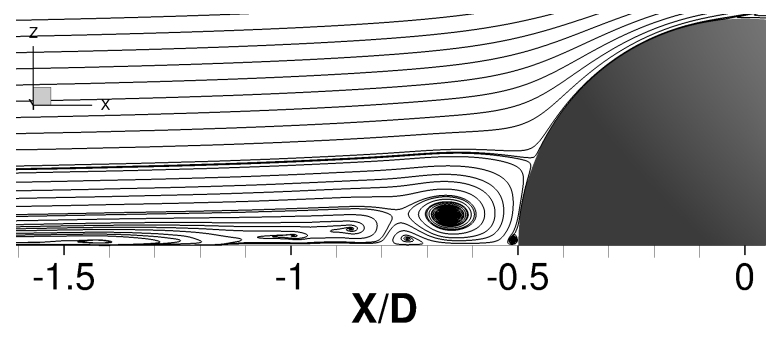

(a) Smagorinsky with $C_{s}=0.1$.

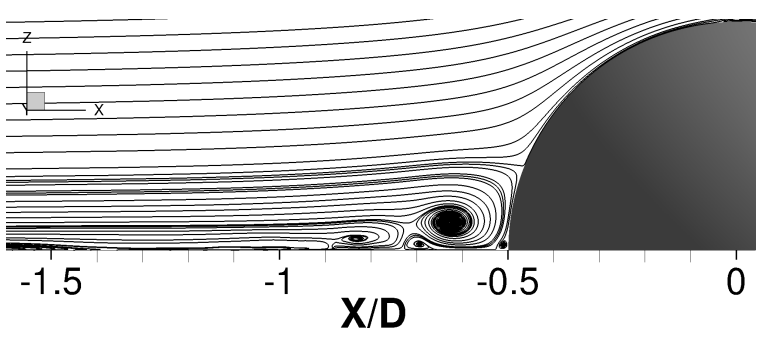

(b) WALE with $C_{W}=0.33$.

Figure B.32: Influence of different SGS models on the flow: Time-averaged streamlines at the symmetry plane $y=0$ (zoom of the region of the horseshoe vortices). 


\section{Appendix C. Influence of the synthetic turbulence inflow data}

To investigate the influence of the synthetic turbulence inflow data on the numerical results, two LES predictions are briefly compared: The setup is identical to the one described in Section 4 except for one simulation carried out without any source terms for the perturbations at $x / D=-1.5$, i.e., a fully steady incoming flow.

Some interesting flow characteristics are plotted for comparison in Fig. C.33. The following observations can be made:

- By considering the synthetic turbulence inflow data the size of the horseshoe vortex in front of the hemisphere is reduced. This can be explained by the higher mixing rate inserted by the synthetic turbulence fluctuations leading to a better agreement with the measurements.

- Due to the application of the inflow data generated by the STIG the flow separation on the hemisphere occurs later: $\theta_{\text {sep }}^{\text {STIG }} \approx 92^{\circ}$ instead of $\theta_{\text {sep }} \approx 96^{\circ}$ without perturbations. Without the STIG data the boundary layer on the hemisphere before the separation line is laminar (see Figs. C.33(c), (e) and (g)).

- Due to the later flow separation the recirculation area is smaller for the case with the synthetic turbulence inflow data (see Figs. C.33(a) and (b)).

This investigation shows that the use of the STIG data, i.e., the turbulence intensity of the oncoming boundary layer and the corresponding flow structures, have a significant impact on the results of the present case. Thus, it is a prerequisite for achieving a good agreement between the numerical results and the measurements. 
Flow, Turbulence and Combustion, vol. 97 (1), pp. 79-119, (2016).

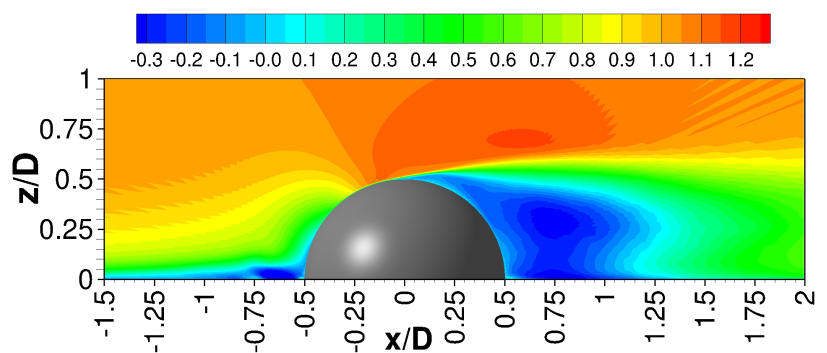

(a) $\bar{u} / U_{\infty}$ (LES without STIG data).

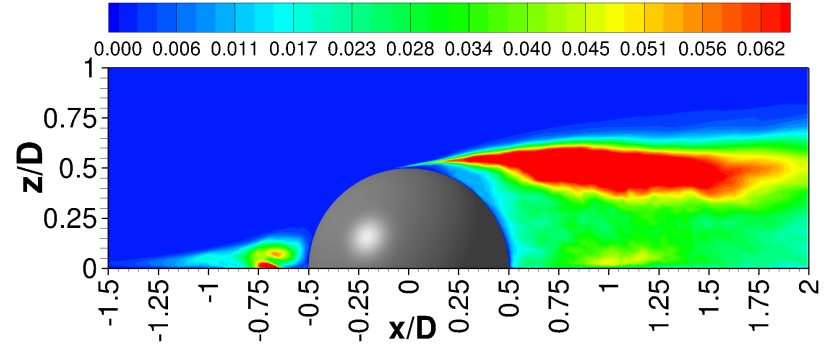

(c) $\overline{u^{\prime} u^{\prime}} / U_{\infty}^{2}$ (LES without STIG data).

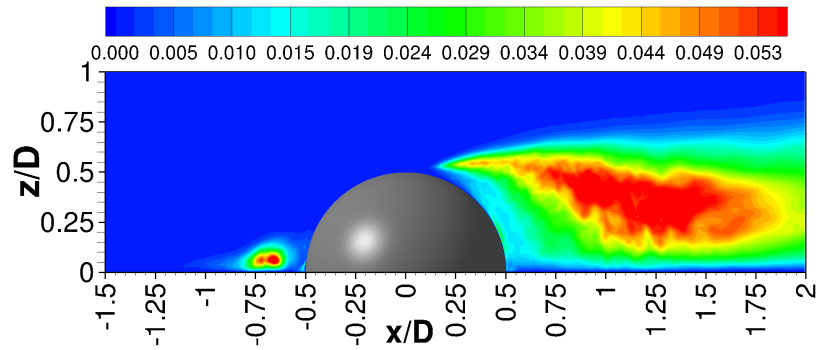

(e) $\overline{w^{\prime} w^{\prime}} / U_{\infty}^{2}$ (LES without STIG data).

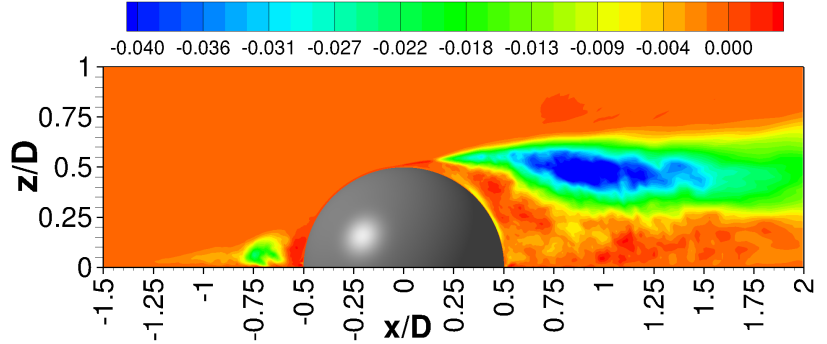

(g) $\overline{u^{\prime} w^{\prime}} / U_{\infty}^{2}$ (LES without STIG data).

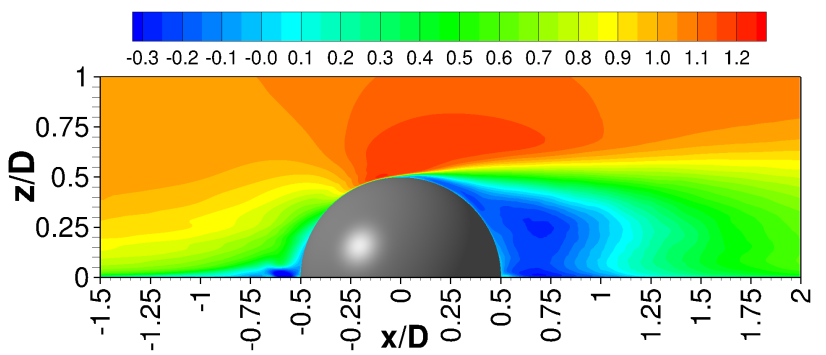

(b) $\bar{u} / U_{\infty}$ (LES with STIG data).

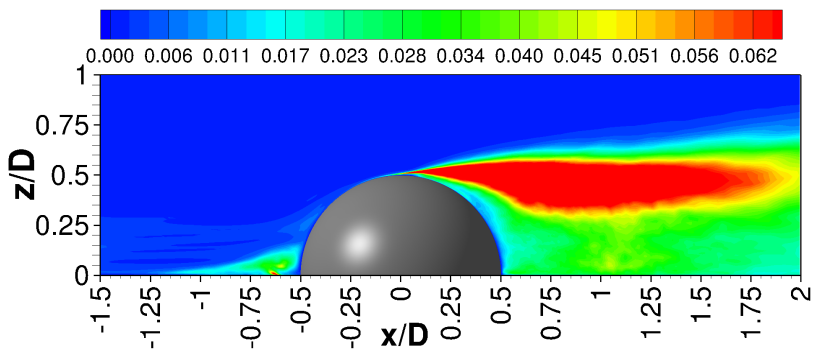

(d) $\overline{u^{\prime} u^{\prime}} / U_{\infty}^{2}$ (LES with STIG data).

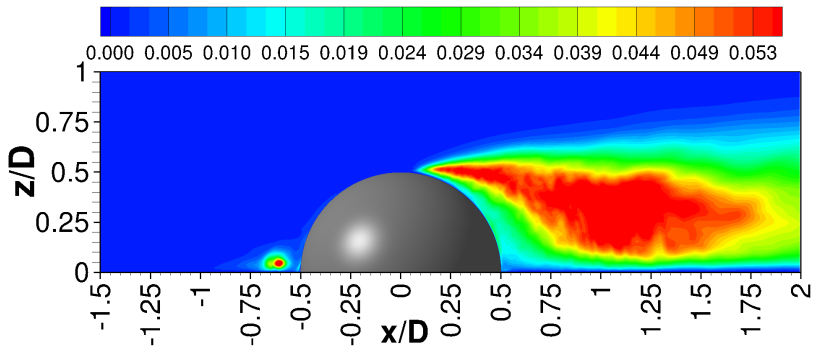

(f) $\overline{w^{\prime} w^{\prime}} / U_{\infty}^{2}$ (LES with STIG data).

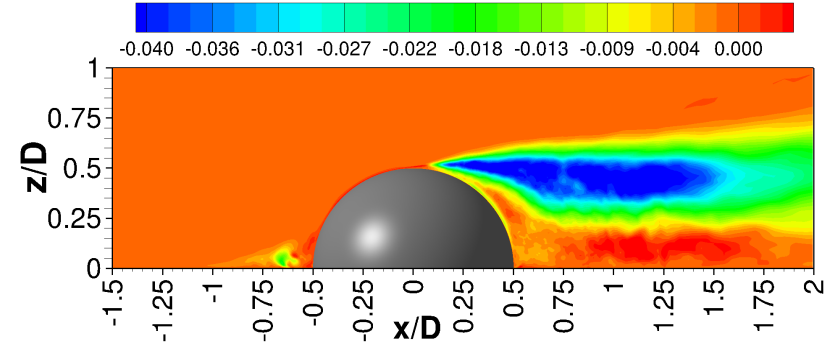

(h) $\overline{u^{\prime} w^{\prime}} / U_{\infty}^{2}$ (LES with STIG data).

Figure C.33: Comparison of numerical time-averaged flow characteristics obtained without or with STIG data. 
Flow, Turbulence and Combustion, vol. 97 (1), pp. 79-119, (2016).

\section{APPENDIX C INFLUENCE OF THE SYNTHETIC TURBULENCE INFLOW DATA}

[1] W. Jacobs, Strömung hinter einem einzelnen Rauhigkeitselement, Ingenieur-Archiv 9 (5) (1938) 343-355.

[2] F. J. Maher, Wind loads on basic dome shapes, Journal of the Structural Division 91 (3) (1965) 219-228.

[3] T. J. Taylor, Wind pressures on a hemispherical dome, Journal of Wind Engineering and Industrial Aerodynamics 40 (2) (1992) 199-213.

[4] S. Taniguchi, H. Sakamoto, M. Kiya, M. Arie, Time-averaged aerodynamic forces acting on a hemisphere immersed in a turbulent boundary, Journal of Wind Engineering and Industrial Aerodynamics 9 (3) (1982) 257-273.

[5] C. M. Cheng, C. L. Fu, Characteristic of wind loads on a hemispherical dome in smooth flow and turbulent boundary layer flow, Journal of Wind Engineering and Industrial Aerodynamics 98 (6) (2010) 328-344.

[6] N. Toy, W. D. Moss, E. Savory, Wind tunnel studies on a dome in turbulent boundary layers, Journal of Wind Engineering and Industrial Aerodynamics 11 (1) (1983) 201-212.

[7] E. Savory, N. Toy, Hemisphere and hemisphere-cylinders in turbulent boundary layers, Journal of Wind Engineering and Industrial Aerodynamics 23 (1986) 345-364.

[8] E. Savory, N. Toy, The separated shear layers associated with hemispherical bodies in turbulent boundary layers, Journal of Wind Engineering and Industrial Aerodynamics 28 (1) (1988) 291-300.

[9] R. Martinuzzi, C. Tropea, The flow around surface-mounted, prismatic obstacles placed in a fully developed channel flow, Journal of Fluids Engineering 115 (1) (1993) 85-92.

[10] R. J. Pattenden, S. R. Turnock, X. Zhang, Measurements of the flow over a low-aspectratio cylinder mounted on a ground plane, Experiments in Fluids 39 (1) (2005) 10-21.

[11] N. Tamai, T. Asaeda, N. Tanaka, Vortex structures around a hemispheric hump, Boundary-Layer Meteorology 39 (3) (1987) 301-314.

[12] M. S. Acarlar, C. R. Smith, A study of hairpin vortices in a laminar boundary layer. Part 1. Hairpin vortices generated by a hemisphere protuberance, Journal of Fluid Mechanics 175 (1987) $1-41$.

[13] J. L. Bennington, Effects of various shaped roughness elements in two-dimensional high Reynolds number turbulent boundary layers, Master thesis, Virginia Polytechnic Institute and State University, Blacksburg, VA (2004).

[14] R. L. Simpson, C. H. Long, G. Byun, Study of vortical separation from an axisymmetric hill, International Journal of Heat and Fluid Flow 23 (5) (2002) 582-591.

[15] G. Byun, R. L. Simpson, Structure of three-dimensional separated flow on an axisymmetric bump, AIAA Journal 44 (5) (2006) 999-1008.

[16] G. Byun, R. L. Simpson, Surface-pressure fluctuations from separated flow over an axisymmetric bump, AIAA Journal 48 (10) (2010) 2397-2405. 
Flow, Turbulence and Combustion, vol. 97 (1), pp. 79-119, (2016).

\section{APPENDIX C INFLUENCE OF THE SYNTHETIC TURBULENCE INFLOW DATA}

[17] M. A. Yaghoubi, Air flow patterns around domed roof buildings, Renewable Energy 1 (3) (1991) 345-350.

[18] T. Tamura, K. Kuwahara, M. Suzuki, Numerical study of wind pressures on a domed roof and near wake flows, Journal of Wind Engineering and Industrial Aerodynamics 36 (1990) 1001-1010.

[19] M. Manhart, Vortex shedding from a hemisphere in a turbulent boundary layer, Theoretical and Computational Fluid Dynamics 12 (1) (1998) 1-28.

[20] R. N. Meroney, C. W. Letchford, P. P. Sarkar, Comparison of numerical and wind tunnel simulation of wind loads on smooth, rough and dual domes immersed in a boundary layer, Wind and Structures 5 (2-4) (2002) 347-358.

[21] D. C. Wilcox, Turbulence Modeling for CFD, 2nd Edition, DCW Industries, Inc., La Cañada CA, 1998.

[22] P. R. Spalart, S. R. Allmaras, A one-equation turbulence model for aerodynamic flows., AIAA Journal 94 (1992) 92-439.

[23] N. Kharoua, L. Khezzar, Large-eddy simulation study of turbulent flow around smooth and rough domes, Proceedings of the Institution of Mechanical Engineers, Part C: Journal of Mechanical Engineering Science 227 (12) (2013) 2686-2700.

[24] M. García-Villalba, N. Li, W. Rodi, M. A. Leschziner, Large-eddy simulation of separated flow over a three-dimensional axisymmetric hill, Journal of Fluid Mechanics 627 (2009) $55-96$.

[25] M. M. Tavakol, M. Yaghoubi, M. Masoudi Motlagh, Air flow aerodynamic on a wallmounted hemisphere for various turbulent boundary layers, Experimental Thermal and Fluid Science 34 (5) (2010) 538-553.

[26] V. Yakhot, S. A. Orszag, S. Thangam, T. B. Gatski, C. G. Speziale, Development of turbulence models for shear flows by a double expansion technique, Physics of Fluids 4 (7) (1992) 1510-1520.

[27] M. M. Tavakol, O. Abouali, M. Yaghoubi, Large eddy simulation of turbulent flow around a wall mounted hemisphere, Applied Mathematical Modelling 39 (13) (2015) 3596-3618.

[28] F. Nicoud, F. Ducros, Subgrid-scale stress modelling based on the square of the velocity gradient tensor, Flow, Turbulence and Combustion 62 (3) (1999) 183-200.

[29] M. Germano, U. Piomelli, P. Moin, W. H. Cabot, A dynamic subgrid-scale eddy viscosity model, Physics of Fluids A 3 (1991) 1760-1765.

[30] W.-W. Kim, S. Menon, Application of the localized dynamic subgrid-scale model to turbulent wall-bounded flows, AIAA Paper No. AIAA-97-0210.

[31] E. Sergent, Vers une methodologie de couplage entre la simulation des grandes echelles et les modeles statistiques, Ph.D. thesis, Ecully, Ecole Centrale de Lyon (2002).

[32] J. Counihan, Adiabatic atmospheric boundary layers: A review and analysis of data from the period 1880-1972, Atmospheric Environment (1967) 9 (10) (1975) 871-905. 
Flow, Turbulence and Combustion, vol. 97 (1), pp. 79-119, (2016).

\section{APPENDIX C INFLUENCE OF THE SYNTHETIC TURBULENCE INFLOW DATA}

[33] R. J. Adrian, C. S. Yao, Power spectra of fluid velocities measured by laser-Doppler velocimetry, Experiments in Fluids 5 (1) (1986) 17-28.

[34] L. H. Benedict, H. Nobach, C. Tropea, Estimation of turbulent velocity spectra from laser-Doppler data, Measurement Science and Technology 11 (8) (2000) 1089-1104.

[35] P. M. T. Broersen, S. de Waele, R. Bos, The accuracy of time series analysis for laserDoppler velocimetry, in: Proceedings of the 10th International Symposium on Application of Laser Techniques to Fluid Mechanics, Lisbon, Portugal, 2000.

[36] T. V. Lawson, Methods of producing velocity profiles in wind tunnels, Atmospheric Environment (1967) 2 (1) (1968) 73-76.

[37] J. Counihan, An improved method of simulating an atmospheric boundary layer in a wind tunnel, Atmospheric Environment (1967) 3 (2) (1969) 197-214.

[38] J. E. Sargison, G. J. Walker, V. Bond, G. Chevalier, Experimental review of devices to artificially thicken wind tunnel boundary layers, in: M. Behnia, W. Lin, G. D. McBain (Eds.), Proceedings of the Fifteenth Australasian Fluid Mechanics Conference (CD-ROM), The University of Sydney, Sydney, Australia, 2004, AFMC00091.

[39] P. Schlatter, R. Orlu, Q. Li, G. Brethouwer, J. H. M. Fransson, A. V. Johansson, P. H. Alfredsson, D. S. Henningson, Turbulent boundary layers up to $\operatorname{Re}_{\theta}=2500$ studied through simulation and experiment, Physics of Fluids 21 (5) (2009) 51702.

[40] F. Durst, M. Schäfer, A parallel block-structured multigrid method for the prediction of incompressible flows, Int. Journal for Numerical Methods in Fluids 22 (6) (1996) 549-565.

[41] F. Durst, M. Schäfer, K. Wechsler, Efficient simulation of incompressible viscous flows on parallel computers, in: E. H. Hirschel (Ed.), Flow Simulation with High-Performance Computers II, Notes on Numerical Fluid Mechanics, Vol. 52(1), Vieweg, 1996, pp. 87-101.

[42] M. Breuer, G. De Nayer, M. Münsch, T. Gallinger, R. Wüchner, Fluid-structure interaction using a partitioned semi-implicit predictor-corrector coupling scheme for the application of large-eddy simulation, Journal of Fluids and Structures 29 (2012) 107-130.

[43] P. K. Khosla, S. G. Rubin, A diagonally dominant second-order accurate implicit scheme, Computers \& Fluids 2 (2) (1974) 207-209.

[44] J. H. Ferziger, M. Perić, Computational Methods for Fluid Dynamics, 3rd Edition, Springer Berlin, 2002.

[45] C. M. Rhie, W. L. Chow, Numerical study of the turbulent flow past an airfoil with trailing-edge separation, AIAA Journal 21 (11) (1983) 1525-1532.

[46] J. Smagorinsky, General circulation experiments with the primitive equations I: The basic experiment, Monthly Weather Review 91 (3) (1963) 99-165.

[47] D. K. Lilly, A proposed modification of the Germano subgrid-scale closure method, Physics of Fluids A 4 (1992) 633-635.

[48] C. Scheit, K. Nusser, G. Hager, S. Becker, T. Zeiser, G. Wellein, Optimizing the FASTEST-3D CFD code for massive parallelism, in: 26th Int. Conf. on Comp. Fluid Dynamics, ParCFD 2014, Norway, Trondheim, 2014. 
Flow, Turbulence and Combustion, vol. 97 (1), pp. 79-119, (2016).

[49] U. Piomelli, J. R. Chasnov, Large-eddy simulations: Theory and applications, in: M. Hallbäck, D. Henningson, A. Johansson, P. Alfredson (Eds.), Turbulence and Transition Modeling, Kluwer, 1996, pp. 269-331.

[50] M. Klein, A. Sadiki, J. Janicka, A digital filter based generation of inflow data for spatiallydeveloping direct numerical or large-eddy simulations, Journal of Computational Physics 186 (2003) 652-665.

[51] T. S. Lund, X. Wu, K. D. Squires, Generation of turbulent inflow data for spatiallydeveloping boundary layer simulations, Journal of Computational Physics 140 (1998) 223258.

[52] S. Schmidt, M. Breuer, Extended synthetic turbulence inflow generator within a hybrid LES-URANS methodology for the prediction of non-equilibrium wall-bounded flows, Flow, Turbulence and Combustion 95 (4) (2015) 669-707.

[53] S. Schmidt, M. Breuer, Application and extension of a synthetic turbulence inflow generator within a hybrid LES-URANS methodology, in: J. Fröhlich, H. Kuerten, B. J. Geurts, V. Armenio (Eds.), ERCOFTAC Series, Direct and Large-Eddy Simulation X, 10th Int. ERCOFTAC Workshop on Direct and Large-Eddy Simulation: DLES-10, Limassol, Cyprus, May 27-29, 2015, Springer Science+Business Media B.V., 2016, to appear.

[54] H. Sakamoto, M. Arie, Vortex shedding from a rectangular prism and a circular cylinder placed vertically in a turbulent boundary layer, Journal of Fluid Mechanics 126 (1983) $147-165$.

[55] S. Okamoto, Y. Sunabashiri, Vortex shedding from a circular cylinder of finite length placed on a ground plane, Journal of Fluids Engineering 114 (4) (1992) 512-521.

[56] C. J. Baker, The turbulent horseshoe vortex, Journal of Wind Engineering and Industrial Aerodynamics 6 (1) (1980) 9-23. 